\title{
March 1999 Working Group Meeting on Heavy Vehicle Aerodynamic Drag: Presentations and Summary of Comments and Conclusions
}

\author{
Rose McCallen \\ Kambiz Salari \\ Fred Browand \\ Mark Brady \\ Jim Ross
}

March 1999

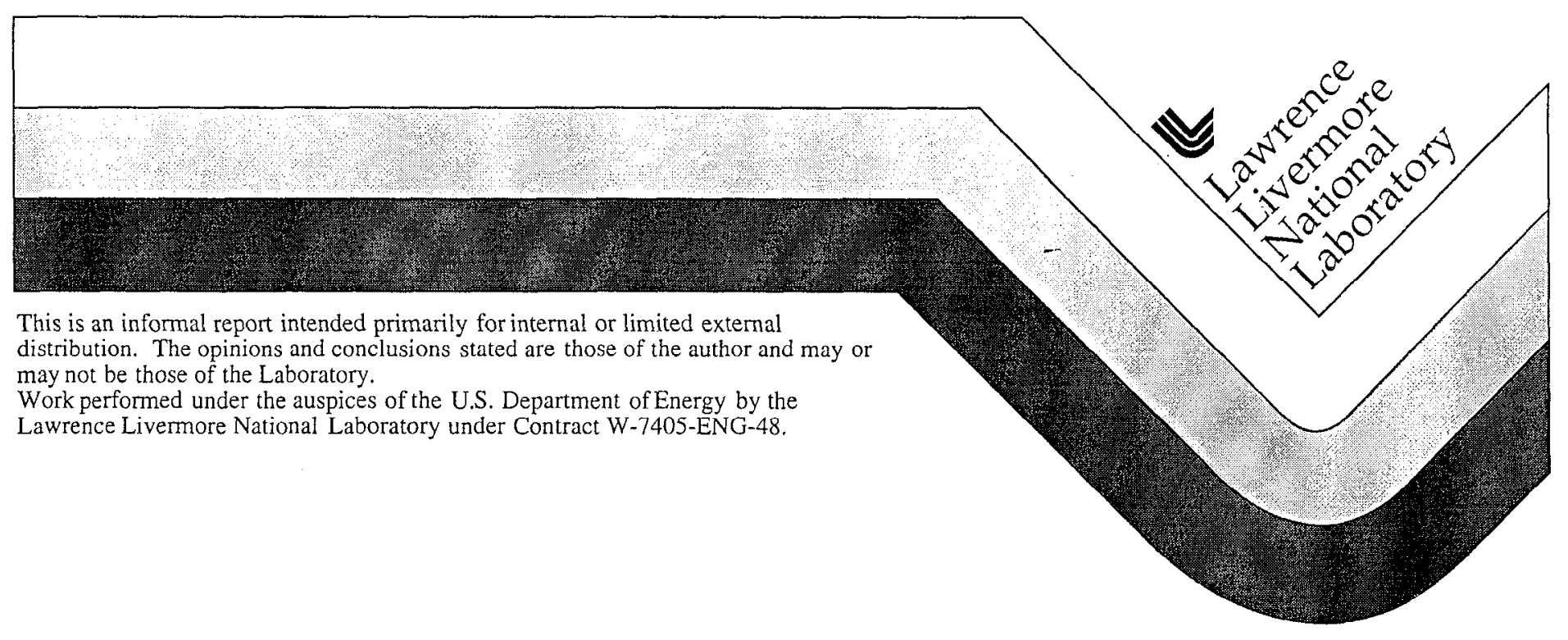




\section{DISCLAIMER}

This document was prepared as an account of work sponsored by an agency of the United States Government. Neither the United States Government nor the University of California nor any of their employees, makes any warranty, express or implied, or assumes any legal liability or responsibility for the accuracy, completeness, or usefulness of any information, apparatus, product, or process disclosed, or represents that its use would not infringe privately owned rights. Reference herein to any specific commercial product, process, or service by trade name, trademark, manufacturer, or otherwise, does not necessarily constitute or imply its endorsement, recommendation, or favoring by the United States Government or the University of California. The views and opinions of authors expressed herein do not necessarily state or reflect those of the United States Government or the University of California, and shall not be used for advertising or product endorsement purposes.

This report has been reproduced directly from the best available copy.

Available to DOE and DOE contractors from the Office of Scientific and Technical Information

P.O. Box 62, Oak Ridge, TN 37831

Prices available from (615) 576-8401, FTS 626-8401

Available to the public from the

National Technical Information Service

U.S. Department of Commerce

5285 Port Royal Rd.,

Springfield, VA 22161 


\title{
March 1999 \\ Working Group Meeting on \\ Heavy Vehicle Aerodynamic Drag:
}

\section{Presentations and Summary of Comments and Conclusions}

Jointly written by

\author{
Lawrence Livermore National Laboratory \\ Sandia National Laboratories \\ University of Southern California \\ California Institute of Technology \\ NASA Ames Research Center
}

\section{Introduction}

A Working Group Meeting on Heavy Vehicle Aerodynamic Drag was held at Lawrence Livermore National Laboratory, Livermore, California on March 11, 1999. The purpose of the meeting was to present technical details on the experimental and computational plans and approaches and provide an update on progress in obtaining experimental results, model developments, and simulations. The focus of the meeting was a review of the experimental results for the integrated tractor-trailer benchmark geometry called the Sandia Model in the NASA Ames $7 \mathrm{ft}$ x $10 \mathrm{ft}$ wind tunnel. The present and projected budget and funding situation was also discussed.

Presentations were given by representatives from the Department of Energy (DOE) Office of Transportation Technology Office of Heavy Vehicle Technology (OHVT), Lawrence Livermore National Laboratory (LLNL), Sandia National Laboratories (SNL), University of Southern California (USC), California Institute of Technology (Caltech), and NASA Ames Research Center. 
This report contains the technical presentations (viewgraphs) delivered at the Meeting, briefly summarizes the comments and conclusions, and outlines the future action items.

\section{Overview of the Project, Current Funding, and Future Workshop}

An overview of the project was presented by Rose McCallen of LLNL. The viewgraphs are enclosed. Budget issues were presented as well as the project calendar of events and milestones.

It was emphasized that the program deliverables are being met only because of the team's success in leveraging funds from internal research support (e.g., LDRD and Tech Base at the National Labs) and the support of other agencies (e.g., DOD, Caltrans, NSF, ASCI) for related work. It was noted that the current budget does not provide funds for the Fall 99 Workshop. LLNL has set aside some of its funding so that we can make commitments to a location and date. Pending SAE approval, the Workshop will be scheduled in conjunction with the SAE Truck and Bus Conference, Detroit, Michigan in November 1999.

Richard Wares of DOE OHVT emphasized the importance of industries positive support for this project. It is also important for the team to keep DOE informed of the past, present, and future significant deliverables, identifying our success in using leveraged funds to achieve these accomplishments, as we have done.

\section{NASA's 7-ft x 10-ft Wind Tunnel Tests}

Jim Ross of NASA Ames presented (with viewgraphs and in computer animation format) our first look at the experimental data on the Sandia Model (a 1/8 scale model) in the $7 \mathrm{ft} x$ $10 \mathrm{ft}$ wind tunnel. In fact, the tests at NASA were still underway on the date of the Meeting. The tests were started on February 12th and were expected to continue through March 12,1999 . The purpose of the tests are for validation of the computational fluid dynamics (CFD) models and for further insight into truck flow phenomena. The viewgraph presentation is enclosed.

The tests were lead by Bruce Storms who was responsible for the preliminary data reduction presented at the Meeting. In addition to drag and discrete and unsteady pressure measurements, an entire suite of new and innovative measurement techniques were used. The NASA Instrumentation Group members that successfully executed these tests and their accomplishments are summarized below.

J.T. Heineck and Stephen Walker of NASA Ames gathered particle image velocimetry (PIV) measurements. The PIV measurements were taken in the model wake, providing the three components of velocity in the plane of a laser sheet. This was only the second time that the 3 simultaneous velocity components have been measured successfully using PIV in a production wind tunnel. (The first was made by the NASA Ames team in July of 1998.) PIV data was taken for Reynolds number ( $\mathrm{Re}$ ) of 0.5 million and 2 million based on the trailer width and upstream velocity. In all, more than fifty data sets were collected. (A sample of the PIV results were presented as an animation and not included in the attached viewgraph presentation.) 
NASA's Dave Driver used his state-of-the-art oil film interferometry techniques (OFI) for measuring skin friction and NASA's James Bell performed pressure sensitive paint (PSP) measurements. The OFI technique can supply quantitative time-averaged skin friction measurements in the body wake (on floor) and the PSP measurements provide time-averaged pressures on the body.

Skin friction measurements on the model body were also provided by Taos hot-film system which can detect flow separation, reattachment, and transition. The Taos System scientists provided the sensors, data collection system, and will be performing the data reduction. A total of 60 data positions were used for the hot-film system (see photograph in attached viewgraph presentation).

Continuum Dynamics also provided (free of charge) boattail plates made to fit our model (see photo in presentation). Tests were conducted with and without the boattail plates. The plates produced a $20 \%$ reduction in drag for the model (see plot of drag coefficient versus yaw angle in presentation). A $10 \%$ reduction has been measured on a full size truck at similar spceds.

Several issues or important poinis were raised during the presentation and during the wrap-up discussions later in the day. These are summarized below.

- The drag data for negative yaw was contaminated due to a damaged lever train, but the positive yaw measurements should be correct and adequate (see drag repeatability plot in presentation).

- The PSP data was noisy, but usable (see scatter plot in presentation).

- All other data looks good.

- The PSP downstream camera caused a drag reduction of $4 \%$ because of the change in back pressure.

- Several of the NASA wind tunnel measurements do not agree with those performed at Texas A\&M on the same model (see comparison of drag measurements in presentation). Data corrections made my Texas A\&M are being investigated. The goal is to account for differences and document them.

- The measured inlet profile (see viewgraphs) is measured at the test section entrance for an empty tunnel. Since the front of the model is only about 6 inches from the start of the test section, the inlet profile for the CFD simulations will need to be extrapolated upstream to obtain accurate flow blockage predictions with the model.

- NASA needs to know what format to use in when providing data to the team (e.g., ascii, TechPlot).

\section{Plans for 12' Wind Tunnel Tests}

Jim Ross of NASA Ames presented the motivation and some approach ideas for the $12-\mathrm{ft}$ wind tunnel tests to be conducted in FYOO at NASA Ames. Jim also led a discussion on possibilities for the model choice and flow conditions during the wrap-up discussion at the 
end of the day.

The 12-ft wind tunnel tests are scheduled for February 2000. The purpose of the tests is to examine Re effects up to full-scale on a $1 / 8$ scale model. The truck industry is very interested in these tests because they recognize that there are discrepancies between the Re effects experienced with a full-size truck and that predicted by experiment on scaled down models in wind tunnels. The NASA test will investigate the minimum $R e$ that can be used in a wind tunnel for accurately predicting the drag effects due to various aspects of the geometry. Geometry aspects that might be considered are the gap distance between the tractor and trailer, the underhood cooling flow, the mirror design, boattail plates, and methods of base drag reduction.

With NASA's ability to acquire detailed unsteady data, these tests serve the dual purpose of being very useful for code validation. However, the CFD team members would then prefer that a simplified body geometry be investigated rather than a more realistic, detailed truck model. It is also anticipated that this data will be used by industry to compare to the simulations generated with commercial tools and a simplified geometry may also be preferred. However, industry would also like to see data for the detailed model for further understanding of the flow phenomena with realistic truck features. No clear direction was established at the Meeting and further discussion is needed.

\section{USC's Wind Tunnel Tests}

Fred Browand and Mustapha Hammache of USC presented a review of recent, relevant publications, an update on preparation of their facility for truck model testing, and results of recent digital particle image velocimetry (DPIV) measurements. The viewgraph presentation is enclosed.

Fred Browand presented a review of a recent publication on a hybrid Reynolds averaged Navier Stokes (RANS) and large-eddy simulation (LES) approach called detached-eddy simulation (DES) for simulating turbulent flow like that for trucks (see viewgraphs for paper references). DES appears to be promising for large flow simulations at high $\mathrm{Re}$.

Fred also sited references that discuss some general shape guidance for drag reduction and provides insight into what should be expected with the Sandia Body. For example, separation at sharp front edges has a dramatic effect on drag. Fred has found that the Sandia body exhibits separation from the front round corners at low Re (per USC's test results) and thus, experiences an increase in drag, where at higher Re (as in the NASA tests), the drag can actually be reduced. Another publication provided a relationship between forebody drag and base pressure coefficient. This type of relationship could make it possible to draw conclusions on the full-body drag for a tractor and trailer from measurements or simulations of just the cab drag.

Experimental drag measurements for the Sandia Body (a 1/15 scale model) were presented for the tractor/trailer combination with and without a gap. The purpose of the tests are for validation of the CFD models and for further insight into truck flow phenomena. The recent experiments have investigated Re effects on full-body drag as well as the effect 
of separation from the cab corner on full-body drag.

Mustapha Hammache described his DPIV methods, emphasizing the importance of smallscale resolution and maximizing accuracy by reducing the field of observation to achieve higher data resolution. He also presented some recent investigations of vehicle platooning which are relative to our investigation of the flow effects due to tractor/trailer gaps. For platooning, decreasing gap distances causes flow entrainment from the top resulting in increased drag on the forward vehicle, while reducing the drag on the aft vehicle.

Mustapha also spent two weeks at NASA working with J.T. Heineck and others in setup and acquiring the PIV data in the $7-\mathrm{ft} \times 10-\mathrm{ft}$ wind tunnel.

\section{Computational Model Development and Simulations}

An overvicw of the Reynolds-averaged Navier Stokes (RANS) computation being performed by SNL was presented by Kambiz Salari. Current efforts involve the modeling of an experiment performed on the Sandia Model in the Texas A\&M 7-ft x 10-ft wind tunnel during 1995. These RANS simulations include part of the converging section, test section, and part of the expansion region of the tunnel. The tunnel walls are treated as slip boundaries (no penetration). The computational meshes for the RANS simulations range from a coarse mesh of 0.5 million nodes to a medium mesh of 4 million nodes for $R e$ of 1.6 million at 0 and 10 degree yaws. Work has begun on a fine mesh case of 32 million nodes which should improve the ability to capture areas of recirculation and separation on the tractor-trailer. For these calculations an implicit finite-volume compressible flow solver with a one-equation Spalart-Allmaras turbulence model was used. The steady solutions were obtained on a massively parallel machine using 107 and 246 processors for the coarse and medium mesh, respectively. The fine mesh calculation which is under way is using 1414 processors. These solutions will then be used as the initial conditions for a time-accurate RANS calculations.

The large-eddy simulation (LES) approach being used by LLNL was presented by Rose McCallen. This advanced modeling approach is being considered to achieve more accurate simulations with minimum empiricism and thus, reduce experimentation. The flow around a tractor/trailer is time dependent, three-dimensional with a wide range of scales (i.e., the largest scale is on the order of the tuck length and the small scales are smaller than the diameter of a grab handle). The approach and development challenges were presented along with a progress update. LLNL is utilizing an established finite element method where a pressure Poisson equation is solved. (The code development work is funded by LLNL Tech Base funds.) Matrix characteristics and solver implementation issues in a parallel solution environment were discussed. A finite element solver interface (FEI) developed by SNL will be utilized which will provide many state-of-the-art solver options being developed at both SNL and LLNL. Rose also showed a preliminary mesh that demonstrated the benefits of the unstructured grid option.

An LES approach with vortex methods being used by Caltech was presented by Mark Brady. Mark emphasized that this is truly a gridless method (except for the $2 \mathrm{D}$ grid on the vehicle surface). Gridless methods appear to be of particular interest to industry, because 
of the excess amount of time that is usually spent on mesh generation compared to the simulation run time. In addition, with vortex methods, computations are only performed where nonzero vorticity is present (e.g., near body and in wake) thus, reducing computational effort. Mark discussed other developments that reduce the effective operations from an order of $\mathrm{N}^{2}$ to order $\mathrm{N}$, where $\mathrm{N}$ is the number of computational elements (i.e., vortex packets) which move with the fluid. Mark provided an update on their progress and planned future work. The fast algorithms have been implemented and an advanced LES approach has been developed and will be implemented. Generation of surface grids for complex geometries needs to be implemented and is being worked on for solution of the Sandia Body.

\section{The Multi-Year Program Plan}

Part of the afternoon discussion was on the multi-year program plan (MYPP). It is anticipated that DOE will need any modifications or additions made to the MYPP prior to the Program review in April.

One suggestion of interest was the expansion of the MYPP to include thermal management (e.g., underhood flow and cooling flows) as well as aerodynamic management with an analysis approach, not a design approach. The thermal and aero management are coupled. For example, the underhood flow can have a significant effect on the body drag. With this addition, the MYPP could include a more thorough system integration analysis. It is believed that industry would welcome this addition.

Another possibility for expansion of the MYPP would be to investigate active control devices to reduce drag or to provide additional download for braking. Such devices might include Stratford shaping, and/or the addition of oscillatory momentum inputs.

These modifications to the existing MYPP would require some further discussion and it was not reasonable to believe that it could be included by the April 99 deadline.

\section{Future Meetings}

The team has been invited to present a paper at the SAE Government/Industry Meeting on April 26-28, 1999 in Washington, DC. Plans are for Rose McCallen to present a brief project overview and Jim Ross will present the wind tunnel test data.

The next Working Group Meeting will take place at USC and will again focus on the NASA $7 \mathrm{ft} \times 10 \mathrm{ft}$ wind tunnel tests and USC's experimental effort. This will be held in mid July and hosted by USC. Progress updates on the computational effort will also be presented and discussed at the meeting, as well as a continued discussion on the addition of a thermal management effort to the existing MYPP.

\section{Action Items}

The follow-on prioritized action items with the individuals responsible for the tasks are as 
follows:

1. Complete 7-ft $\times 10$-ft wind tunnel tests, reduce data and analyze, assemble a data report, and distribute data for validation work. (Jim Ross and the NASA Ames experimental team).

2. Provide NASA with the desired data format (ascii or TechPlot). (R. McCallen and K. Salari)

3. Finalize scheduling of Fall 99 Workshop with the SAE Truck and Bus Conference. (R. McCallen)

4. Determine poster and paper presentation options at SAE Truck and Bus Conference. (R. McCallen)

5. Keep Frank Tokarz informed of the past, present, and future significant deliverables, identifying our success in using leveraged funds so that he can adequately represent us at DOE meetings (e.g., OHVT Annual Program Review and Planning Meeting in April). (R. McCallen)

6. Provide DOE with updated budget request by end of March. (R.McCallen)

7. Setup a series of e-mail and conference call discussions in an attempt to determine the pros and cons of the various geometry options for the NASA 12-ft wind tunnel tests currently scheduled for February 2000. (R. McCallen)

8. Try again to setup site visit at Paccar. (R. McCallen)

9. Plan and host next Working Group Meeting at USC. Provide team with agenda at least one week in advance. (Fred Browand and Mustapha Hammache) 
Truck Aero Team Meeting

NASA Ames, CA

March 11, 1999

\section{$\underline{\text { Attendee List }}$}

\begin{tabular}{lll} 
Attendee & Organization & e-mail address \\
\hline Salvador Aceves & LLNL & acevessaborio1@llnl.gov \\
Mark Brady & Caltech & mbrady@caltech.edu \\
Fred Browand & USC & brownad@spock.usc.edu \\
Richard Couch & LLNL & couch1@ Ilnl.gov \\
Mustapha Hammache & USC & hammache@spock.usc.edu \\
Barbara Kornblum & LLNL & kornblum1@llnl.gov \\
Satish Kulkarni & LLNL & kulkarni1@llnl.gov \\
Helen Magann & LLNL & magann2@llnl.gov \\
Rose McCallen & LLNL & mccallen1@llnl.gov \\
Erma Paddack & LLNL & paddack1@llnl.gov \\
Jim Ross & NASA Ames & jcross@mail.arc.nasa.gov \\
Kambiz Salari & SNL & ksalari@sandia.gov \\
Frank Tokarz & LLNL & tokarz1@llnl.gov \\
Kichard Wares & DOE & richard.wares@ee.doe.gov
\end{tabular}




\section{- Agenda - \\ Truck Aero Team Meeting \\ NASA Ames, CA}

March 11, 1999

\section{Purpose of Meeting}

Technical details on experimental and computational plans and approaches

Update on progress - experimental results, model developments, simulations

\section{Introduction}

Introduction

R. McCallen, LLNL

\section{Experimental Work and Progress}

NASA 7'x 10 ' Wind Tunnel Test Results and

Plans for 12' Wind Tunnel Tests

Jim Ross, NASA Ames

Wind Tunnel Tests at USC

Fred Browand and Mustapha Hammache, USC

\section{DOE Report}

Update

Richard Wares, DOE

\section{Computational Work and Progress}

RANS/LES Modeling Progress and Results at SNL

Kambiz Salari, SNL

FEM /LES Development and Modeling Progress at LLNL

Rose McCallen, LLNL

Vortex Method/LES Development and Modeling Progress at Caltech

Mark Brady, Caltech

\section{Wrap-up Discussion}

Discussion on Experiments

Discussion on Computations

Calendar of Near Term Events

Near Term Action Items

Date and Location for Next Meeting 


\title{
Aerodynamic Design of Heavy Vehicles
}

\author{
Overview of Project
}

\author{
Rose McCallen, Ph.D.
}

Lawrence Livermore National Laboratory, Livermore, CA

March 1999 


\section{The truck industry relies on wind tunnel and field experiments for aerodynamic design and analysis.}

Wind Tunnel Testing

Costly detailed models

Testing is expensive

Trial-error approach to determine drag effects due to

- general tractor shape, under-body and underhood flow

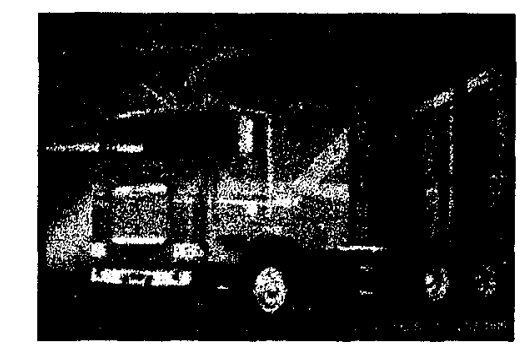

- positioning and shaping of head lamps or turning lights

- mirror and grab handle configurations and positioning

- tractor-trailer gaps and height mismatch

Field Testing

Performed by both manufacturer and fleet operators

Issues

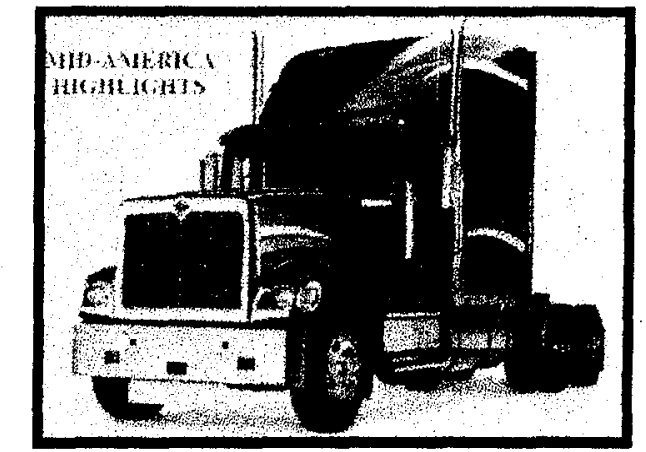

Conventional

A tractor is paired with several different trailers

Almost no aero design interaction between tractor and trailer manufacturers

The effects of design changes on drag are not well understood and computational guidance is needed and welcomed by industry 
The MYPP is based on industry needs and consideration of current technology, funding, and DOE interests.

DOE and National Laboratory interest

Reduce heavy vehicle drag $\rightarrow>$ reduce fuel consumption and emissions

R\&D for DOE programs

Industry needs

Advanced computational tools and experimental methods

- Understand the effects of design changes

- Simulate fully-integrated tractor-trailers

Design improvements for drag reduction

Current technology - CFD is hard!

Reynolds-averaged Navier Stokes (RANS) is common approach

Large-eddy simulation (LES) is in development

DPIV measurements can provide full velocity field measurements

Funding is minimal and we need a plan with a 'near-term impact'

FY99: $\$ 435 K$ ( $=67 \%$ of requested $\$ 635 K$ ) 


\section{The MYPP focuses on development and demonstration of a simulation capability.}

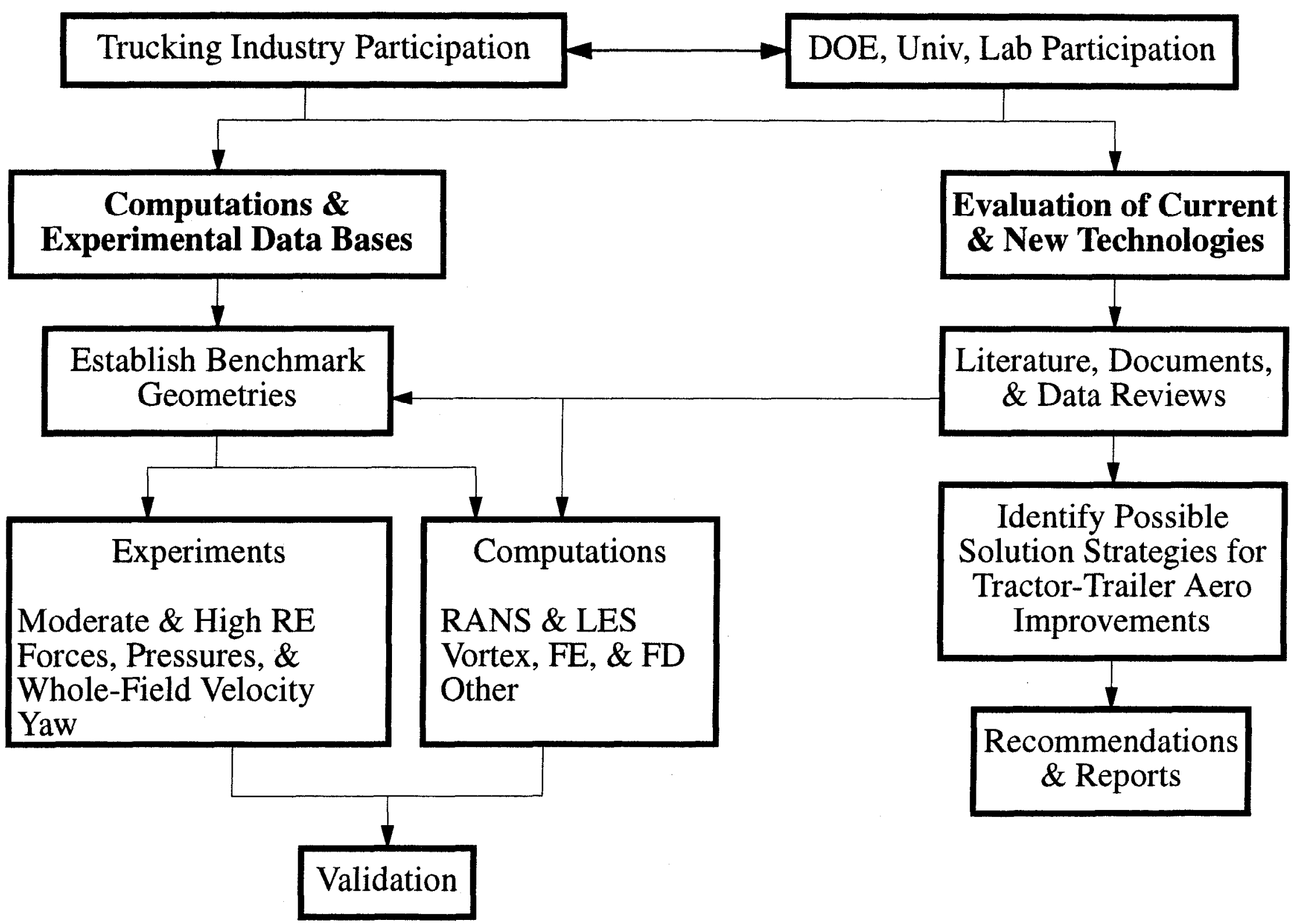




\section{Each organization's contributions are critical to the project's success.}

Computational Modeling

Rose McCallen (PI)

University of California

Lawrence Llvermore

Natlonal Laboratory
Large-Eddy Simulation using

Finite Element Methods
Experimental Modeling

Don McBride

Walt Rutledge

Anthony Leonard

Mark Brady

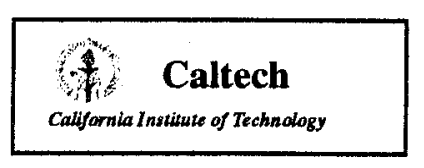

Large-Eddy Simulation using Vortex Methods

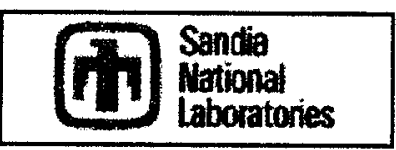

GTS Experiments at Texas A\&M

Kambiz Salari

Walt Rutledge

Don McBride
Fred Browand Mustapha Hammache

\begin{tabular}{|cc|}
\hline USC & UNIVERSITY \\
OF SOUTHERN \\
CALIFORNIA
\end{tabular}

Moderate Speed Experiments in Wind Tunnel
Jim Ross

Bruce Storms, JT Heineck

National

Aeronautics \&

Space

Adm/n/stration

Reynolds-Averaged Modeling using Finite Difference Methods

High Speed Experiments in 7'x10' and 12' Wind Tunnels 


\section{Near-Term Impact: Comparison of RANS and LES and}

detailed experimental verification for a truck problem.

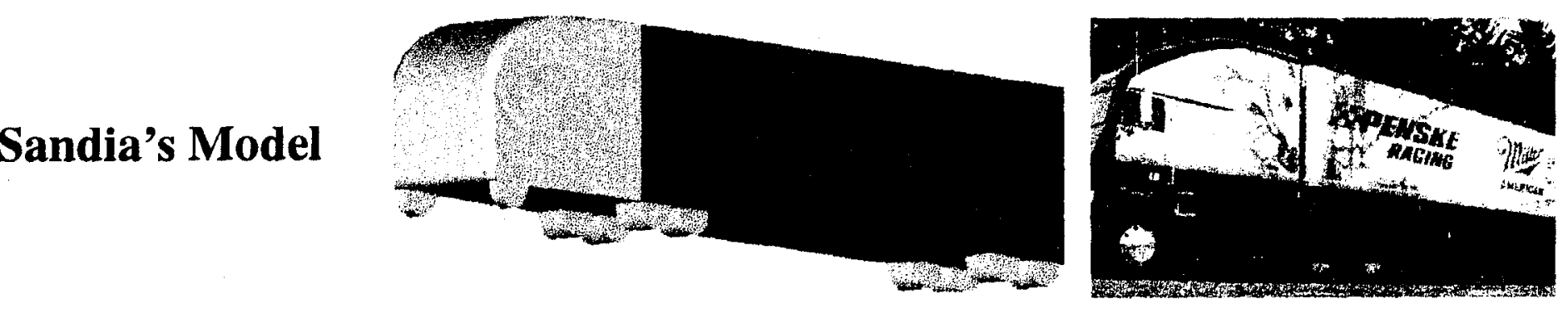

Advantages

Simple geometry with some existing data and some modeling already done The final detail results will be available for comparison to commercial CFD tools

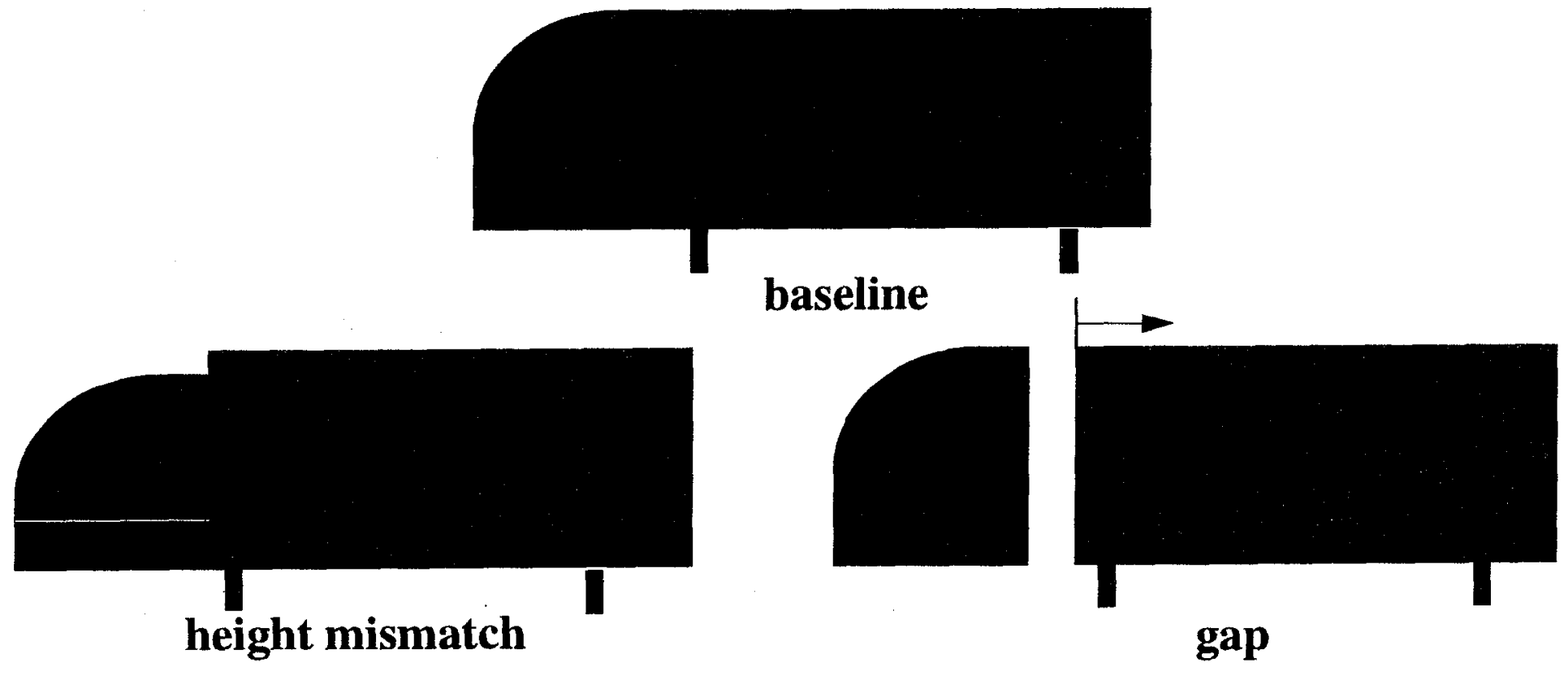




\section{Our near-term tasks have been identified and prioritized.}

Benchmarks

1. Sandia Body

Experiments

- Texas A\&M, $\operatorname{Re}=1,600,000$ (1:8 scale)

- NASA 7'x10', $\operatorname{Re}=\mathbf{2 , 0 0 0 , 0 0 0}$ to lowest $\operatorname{Re}(1: 8$ scale)

- USC wind tunnel, 200,000 < $\operatorname{Re}<400,000$ (1:15 scale) With/without height mismatch and gap

Computations

- RANS for high and low Re (SNL)

- LES for low Re, attempt at high Re (LLNL and Caltech)

2. New Model Design (USC)

3. Navistar's Model for Re sensitivity study

- NASA 12' wind tunnel

$\mathrm{Re}_{\text {max }}=5,000,000$, model with/without components
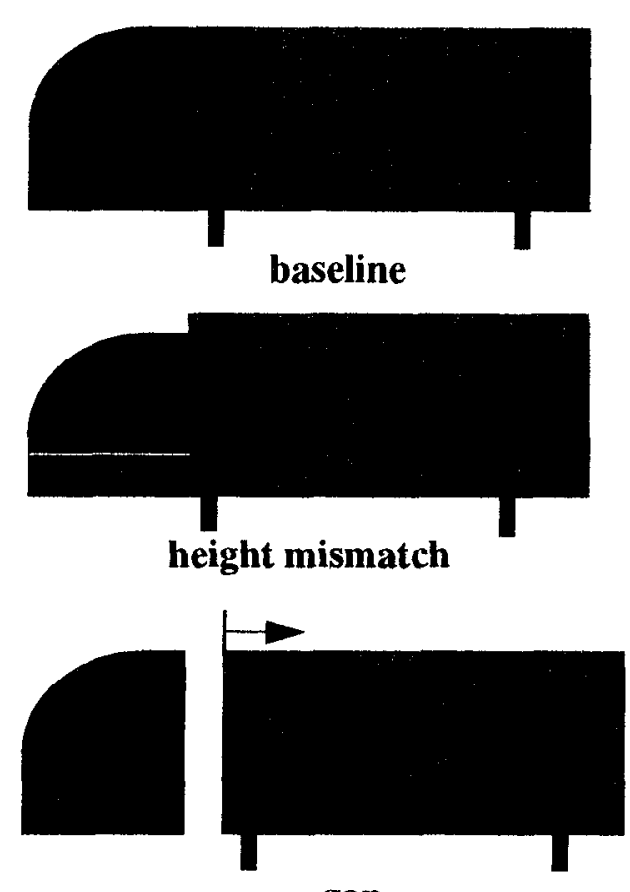

gap

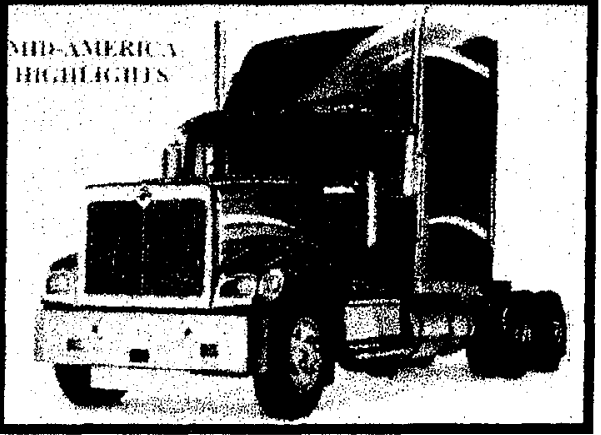




\section{Our budget is not consistent with projected funding.}

\begin{tabular}{|l|c|c|c|c|c|}
\hline & $\begin{array}{c}\text { Computations } \\
\text { \& Experiments }\end{array}$ & $\begin{array}{c}\text { Evaluation of } \\
\text { Current \& New } \\
\text { Technologies }\end{array}$ & Final Report & $\begin{array}{c}\text { Total requested/ } \\
\text { Year }\end{array}$ & $\begin{array}{c}\text { Total received/ } \\
\text { Year }\end{array}$ \\
\hline FY98 & $\$ 276 \mathrm{~K}$ & $\$ 34 \mathrm{~K}$ & & $\$ 310 \mathrm{~K}$ & $\$ 325 \mathrm{~K}$ \\
\hline FY99 & $\$ 630 \mathrm{~K}$ & $\$ 5 \mathrm{~K}$ & & $\$ 635 \mathrm{~K}$ & $\$ 435 \mathrm{~K}$ \\
\hline FY00 & $\$ 1,045 \mathrm{~K}$ & $\$ 188 \mathrm{~K}$ & & $\$ 1,233 \mathrm{~K}$ & \\
\hline FY01 & $\$ 1,095 \mathrm{~K}$ & $\$ 188 \mathrm{~K}$ & & $\$ 1,283 \mathrm{~K}$ & \\
\hline FY02 & $\$ 855 \mathrm{~K}$ & $\$ 161 \mathrm{~K}$ & & $\$ 1016 \mathrm{~K}$ & \\
\hline FY03 & $\$ 818 \mathrm{~K}$ & $\$ 161 \mathrm{~K}$ & & $\$ 979 \mathrm{~K}$ & \\
\hline FY04 & $\$ 120 \mathrm{~K}$ & $\$ 124 \mathrm{~K}$ & $\$ 34 \mathrm{~K}$ & $\$ 278 \mathrm{~K}$ & \\
\hline TOTAL & & & & $\$ 5,734 \mathrm{~K}$ & \\
\hline
\end{tabular}


Funding for FY98 and FY99

\begin{tabular}{|l|c|c|}
\hline & FY 98 & FY 99 \\
\hline LLNL & $\$ 100 K$ & $\$ 170 K^{*}$ \\
\hline SNL & $\$ 100 \mathrm{~K}$ & $\$ 80 \mathrm{~K}$ \\
\hline USC & $\$ 50 \mathrm{~K}$ & $\$ 80 \mathrm{~K}$ \\
\hline Caltech & $\$ 50 \mathrm{~K}$ & $\$ 80 \mathrm{~K}$ \\
\hline NASA & $\$ 25 \mathrm{~K}$ & $\$ 25 \mathrm{~K}(\mathbf{\$ K})$ \\
\hline & & \\
\hline Totals & $\$ 325 \mathrm{~K}$ & $\$ 435 \mathrm{~K}(\mathbf{\$ 4 4 1})$ \\
\hline
\end{tabular}

* Includes project management tasks, LES modeling, and \$15K for workshop. 
It was necessary to leverage other funding sources.

\begin{tabular}{lll}
\hline SNL & - past data obtained at Texas A\&M & Free \\
& - loan of model to NASA & Free \\
& - LES R\&D & LDRD \\
& - computational resources & ASCI \\
USC & - instrumentation & Caltrans, NSF \\
& & \\
Caltech & - LES model development & ASCI, DOD \\
& - computational resources & ASCI, NSF, DOD \\
& & \\
NASA Ames & - 7'x10' wind tunnel tests & Free \\
& - 12' wind tunnel tests & 1/3 Cost \\
& - loan of Navistar's model & Free \\
LLNL & - computational resources & \\
& - LES and code development & ASCI
\end{tabular}




\section{The projected milestones are segregated into benchmark cases with advancing levels of complexity.}

Projected milestones for first four years of project (FY98 through FY01)

\begin{tabular}{|l|c|}
\hline \multicolumn{1}{|c|}{ Task } & Milestone \\
\hline Workshop II & $2 / 98$ \\
\hline MYPP with projected budget and milestones & $5 / 98$ \\
\hline Continued site visits / Working Group Meetings (reports) & $8 / 98,10 / 98,3 / 99$ \\
\hline $\begin{array}{l}\text { Level 1 Benchmarks: Establish generic shapes and outline } \\
\text { test cases for investigation of trailer-tractor height and gap } \\
\text { mismatch }\end{array}$ & $9 / 98$ \\
\hline Level 2 Benchmarks: Establish generic shapes & \\
\hline Test data at moderate Re for Level 1 benchmarks & $9 / 99$ \\
\hline Test data at high Re for Level 1 benchmarks & $11 / 99$ \\
\hline Workshop III & $11 / 99$ \\
\hline $\begin{array}{l}\text { RANS, LES/FEM, LES/Vortex computations of Level 1 } \\
\text { benchmarks at moderate Re }\end{array}$ & $11 / 99$ \\
\hline $\begin{array}{l}\text { RANS, LES/FEM, LES/Vortex computations of Level 1 } \\
\text { benchmarks at high Re }\end{array}$ & $12 / 99$ \\
\hline Test data at moderate and high Re for Level 2 benchmarks & $12 / 00$ \\
\hline
\end{tabular}




\section{We have a full calendar over the next 8 months.}

Web Page (3/99)

Maintain with current information, links, etc.

Progress Report (4/99)

Each organizations input needed

SAE Meeting, Washington, DC (4/99)

Abstract submitted

Need computational/experimental results to present

MYPP (5/99)

Submit updated/final version to DOE (Put design/integration task back in? Edits?)

Working Group Meeting (6/99)

USC/Caltech visit

Progress Report (7/99)

Workshop III, Detroit, MI(11/99)

Defined purpose, agenda, dates, etc.

Waiting for SAE approval

Determine invited speaker, advertise, prepare presentations, etc. 


\section{GTS Model Experiment in the $7 \times 10$}

\section{Bruce Storms}

J.T. Heineck

Steve Walker

James Bell

Dave Driver etc... 


\section{Test Summary}

- Test started February 8

- Test complete March 12

- Objective - CFD validation

- Principle measurements

- Drag and discrete pressure measurements

- Pressure-Sensitive Paint (PSP)

- Unsteady pressure (one point on rear "door")

- Skin friction (oil film interferometry)

- Particle Imaging Velocimetry

- Transition (surface hot films) 


\section{Summary of Results}

- Drag data contaminated by fouling and sloppy lever train

- One of the side-force scales has loose bearing

- Drag data for negative yaw is poor

- Positive yaw data is good

- PSP data noisy but usable - at very low end of measurable pressure variations

- All other data looks good 


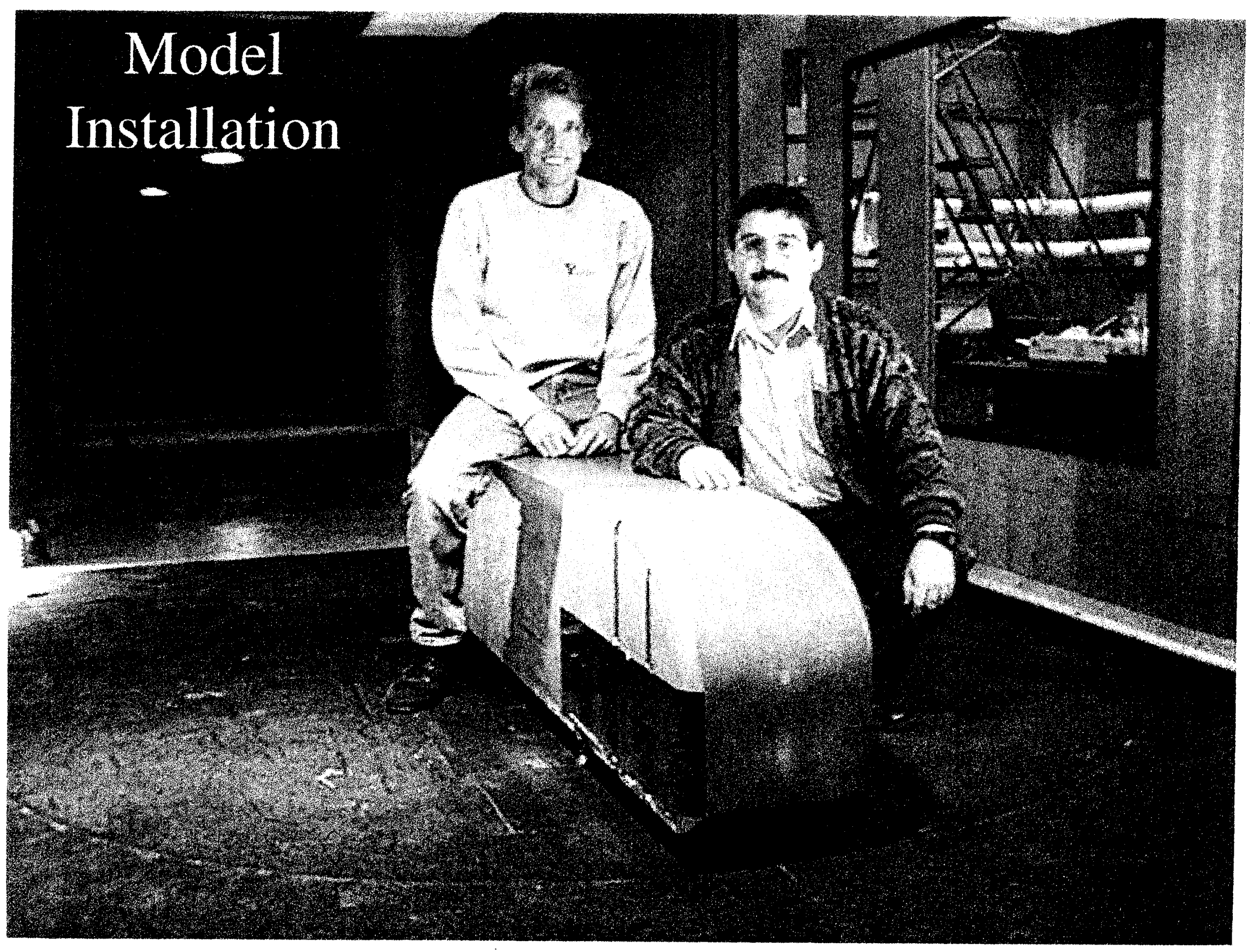




\section{GTS Model in 7x10}
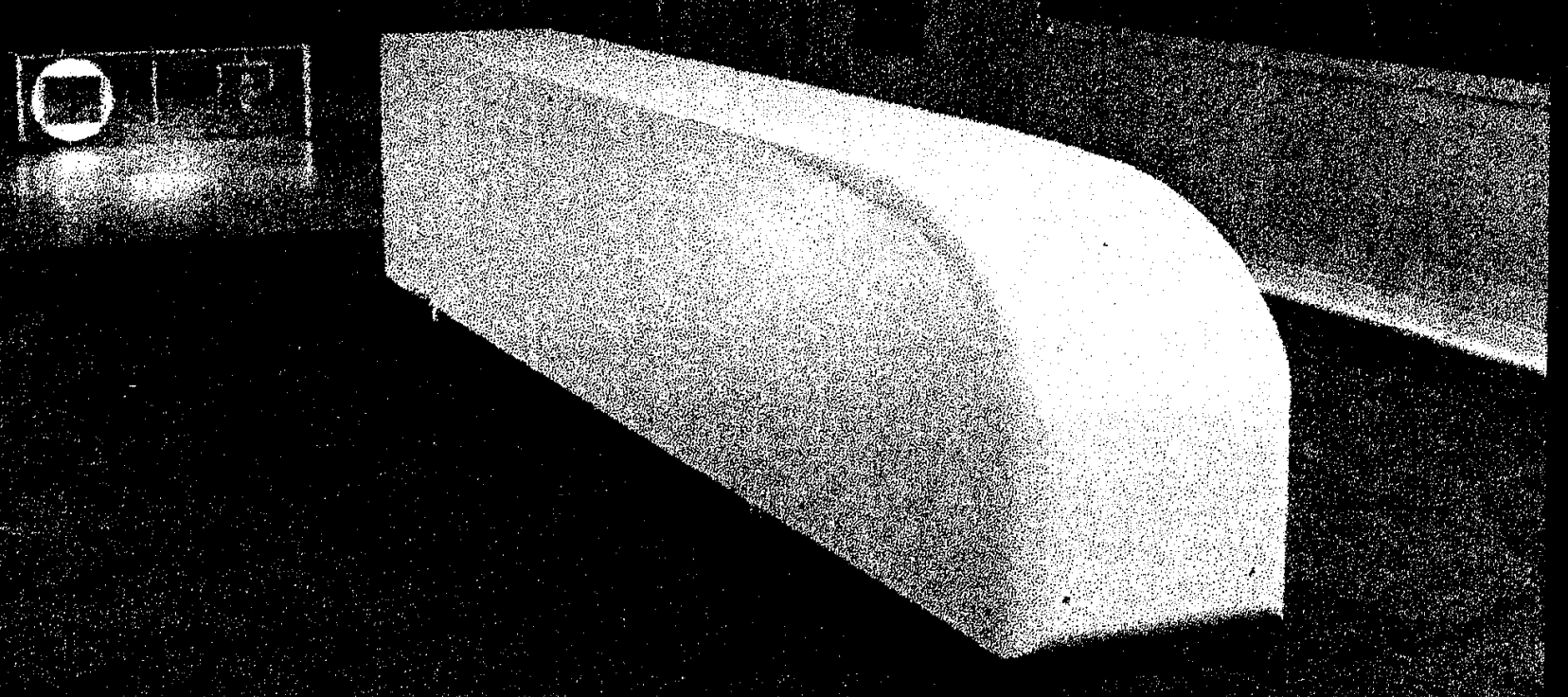


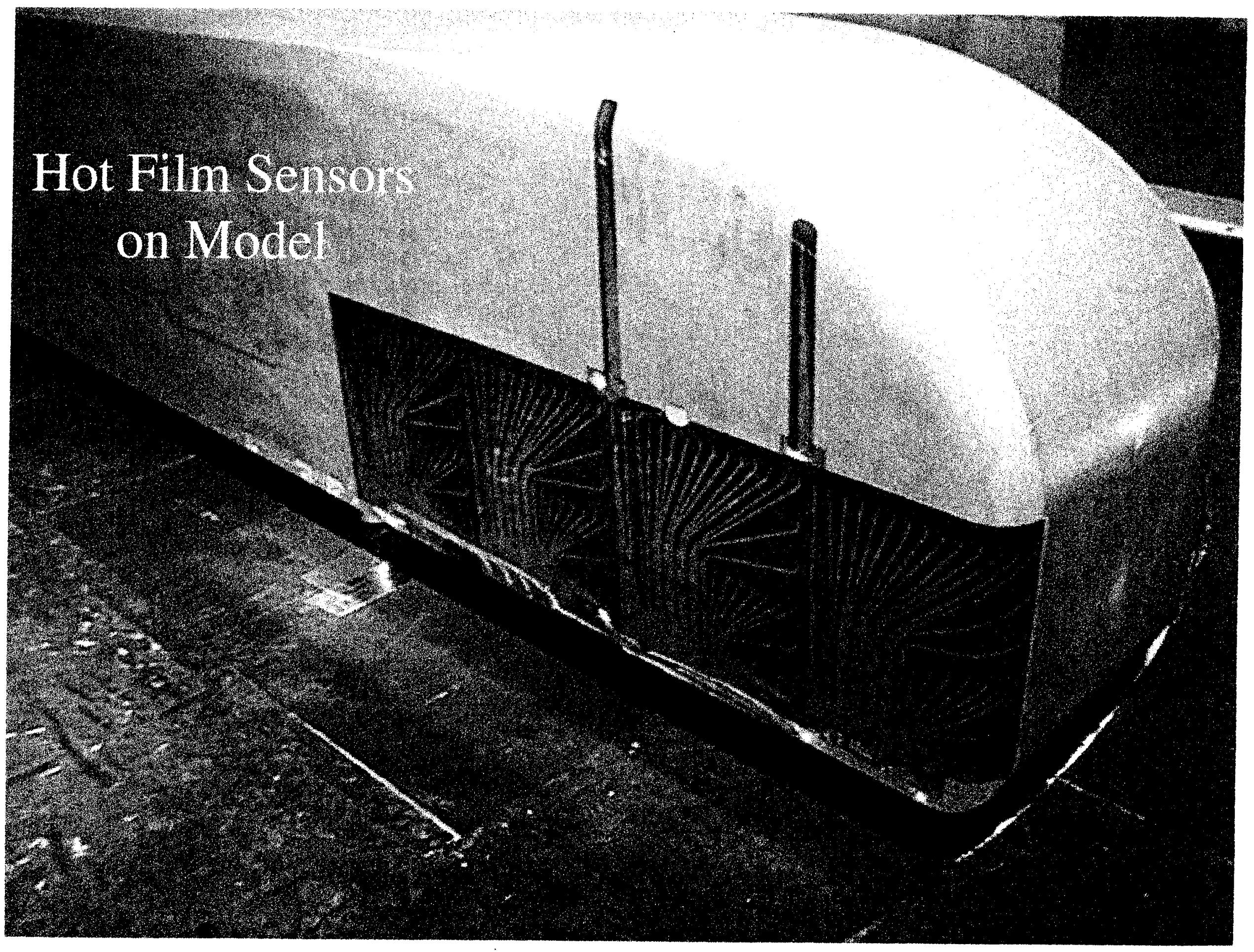




\section{Continuum \\ Dynamics \\ Boattail Plates \\ Installed on \\ Model}

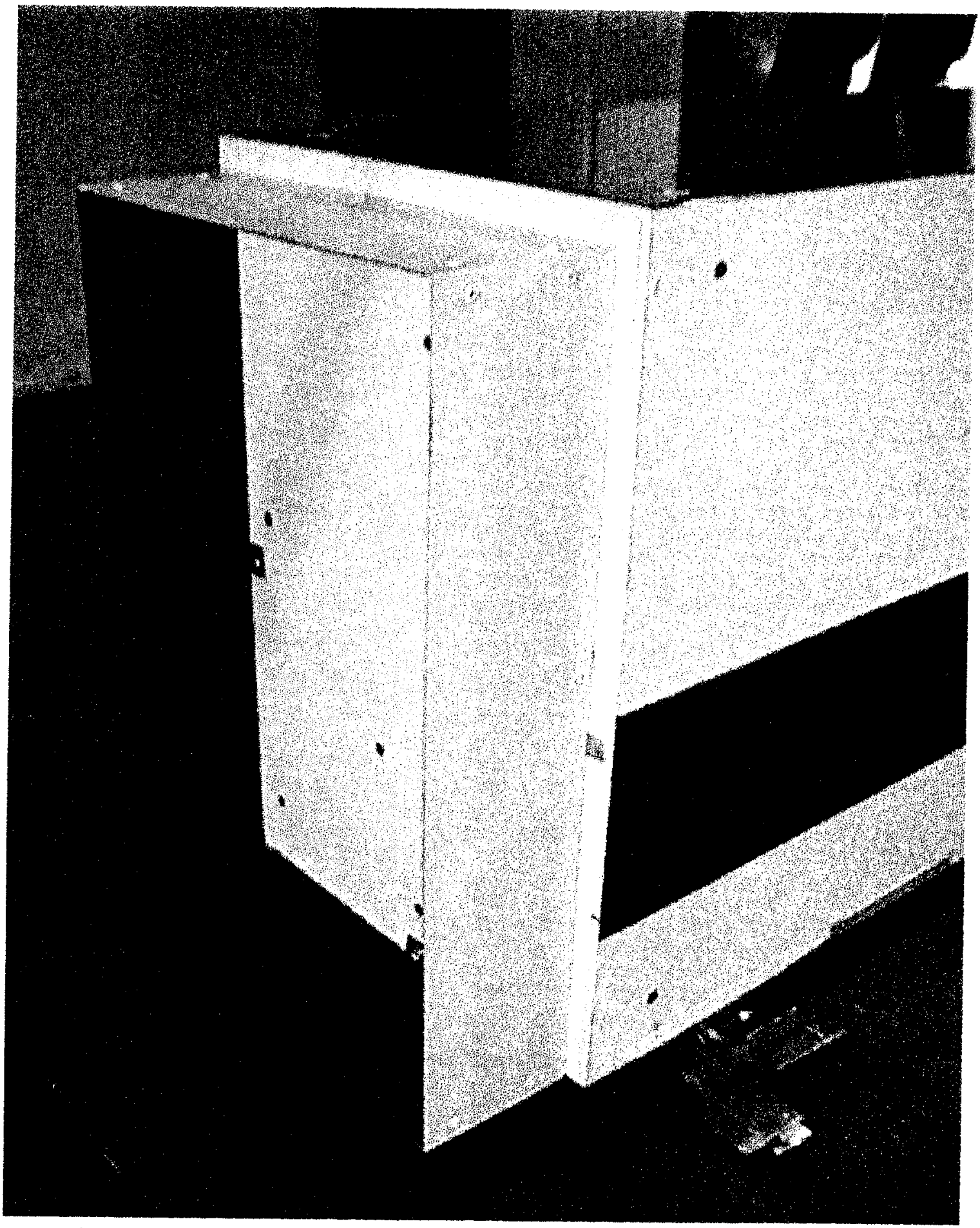




\section{Floor Boundary Layer Profile in 7x10}

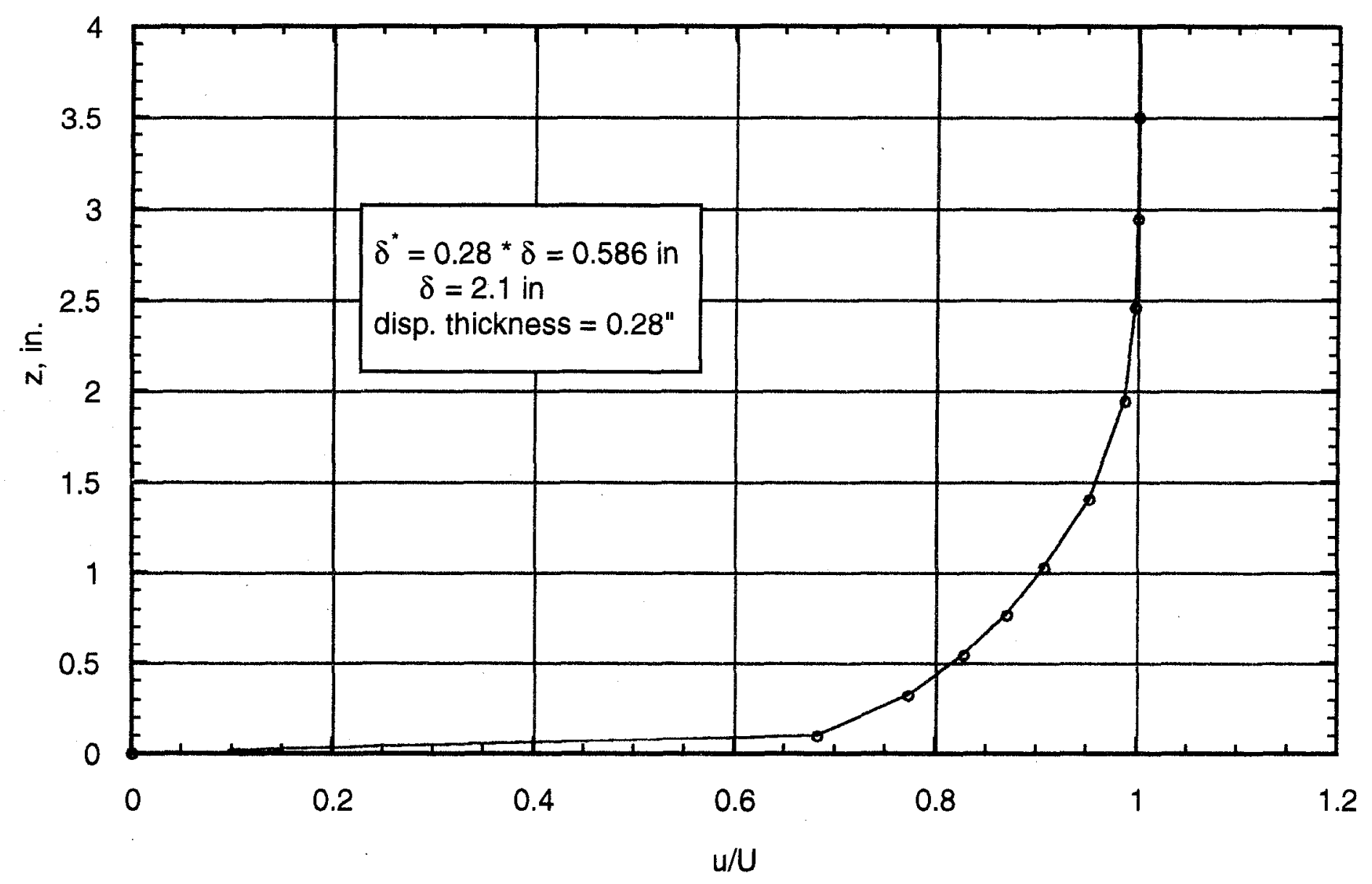




\section{Drag Repeatability}

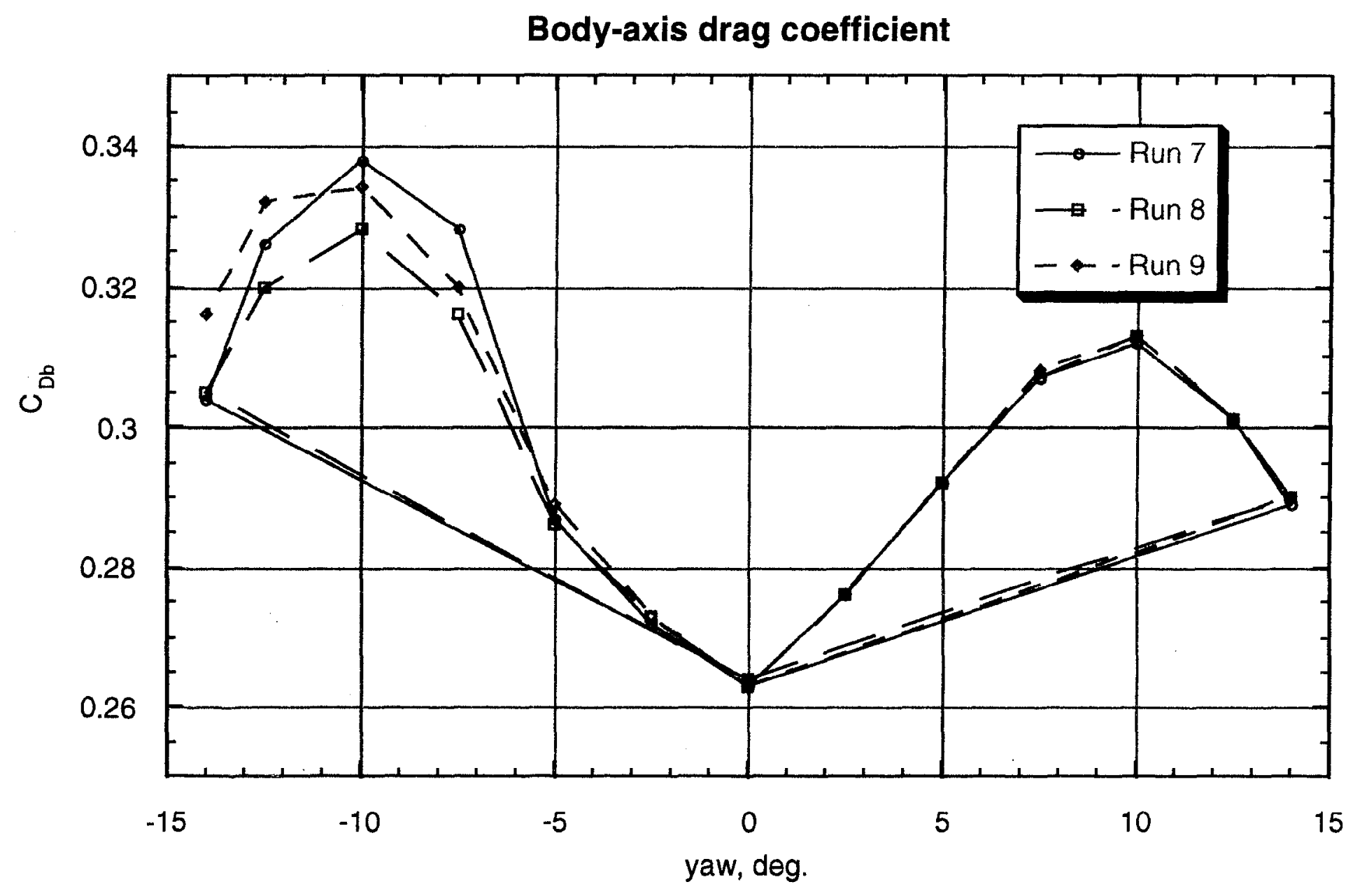




\section{Comparison of 7x10 and Texas A\&M Drag Results}

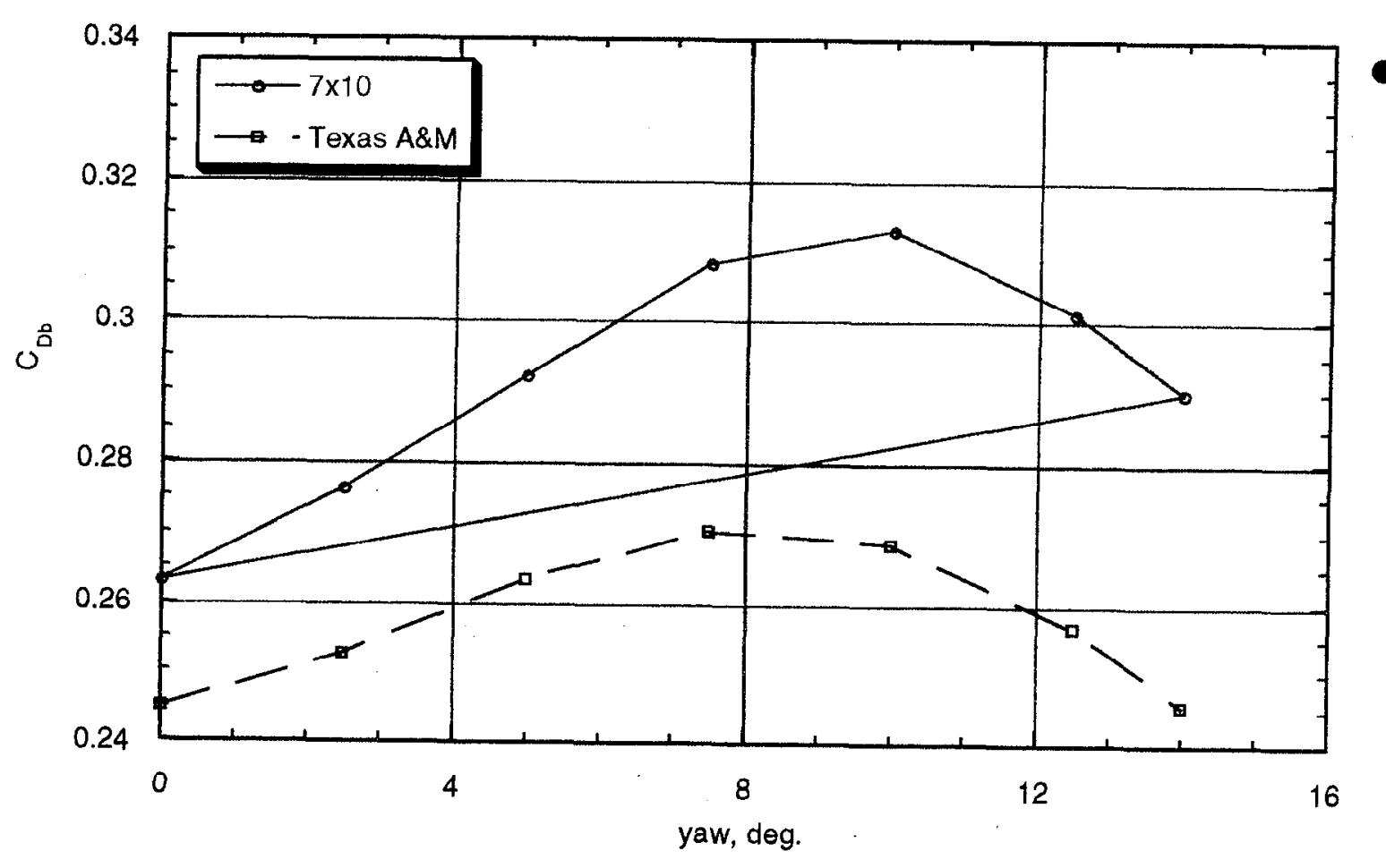

- Differences

- Corrections applied at A\&M?

- Location of static reference pressure ring 


\section{Effect of Boattail Plates on Drag}

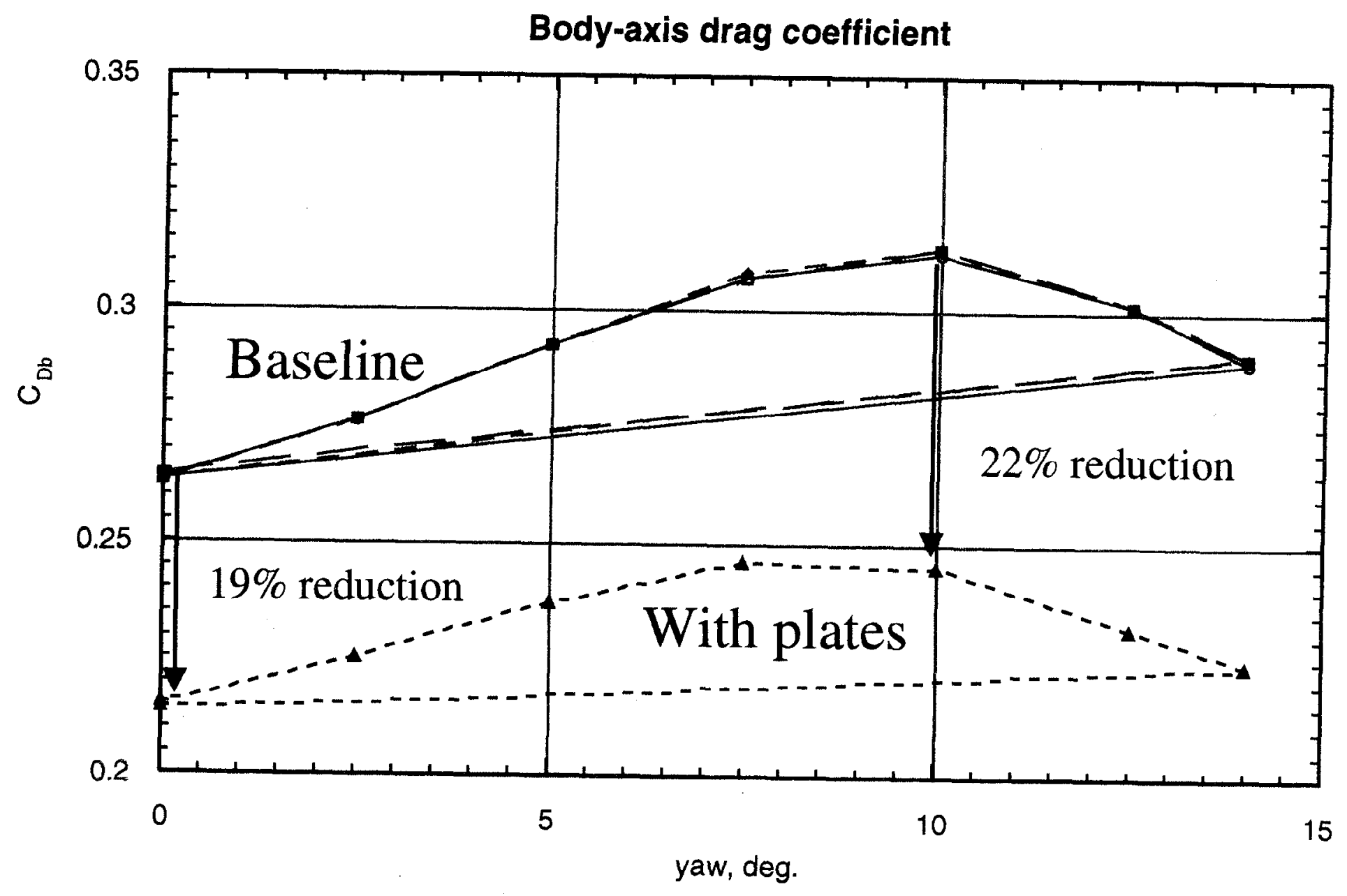




\section{PSP Results on GTS Model in Ames 7x10

$$
\mathrm{Q}=88 \mathrm{psf}, \mathrm{Yaw}=10^{\circ}
$$

(View shows suction surface of model)

Flow

Direction
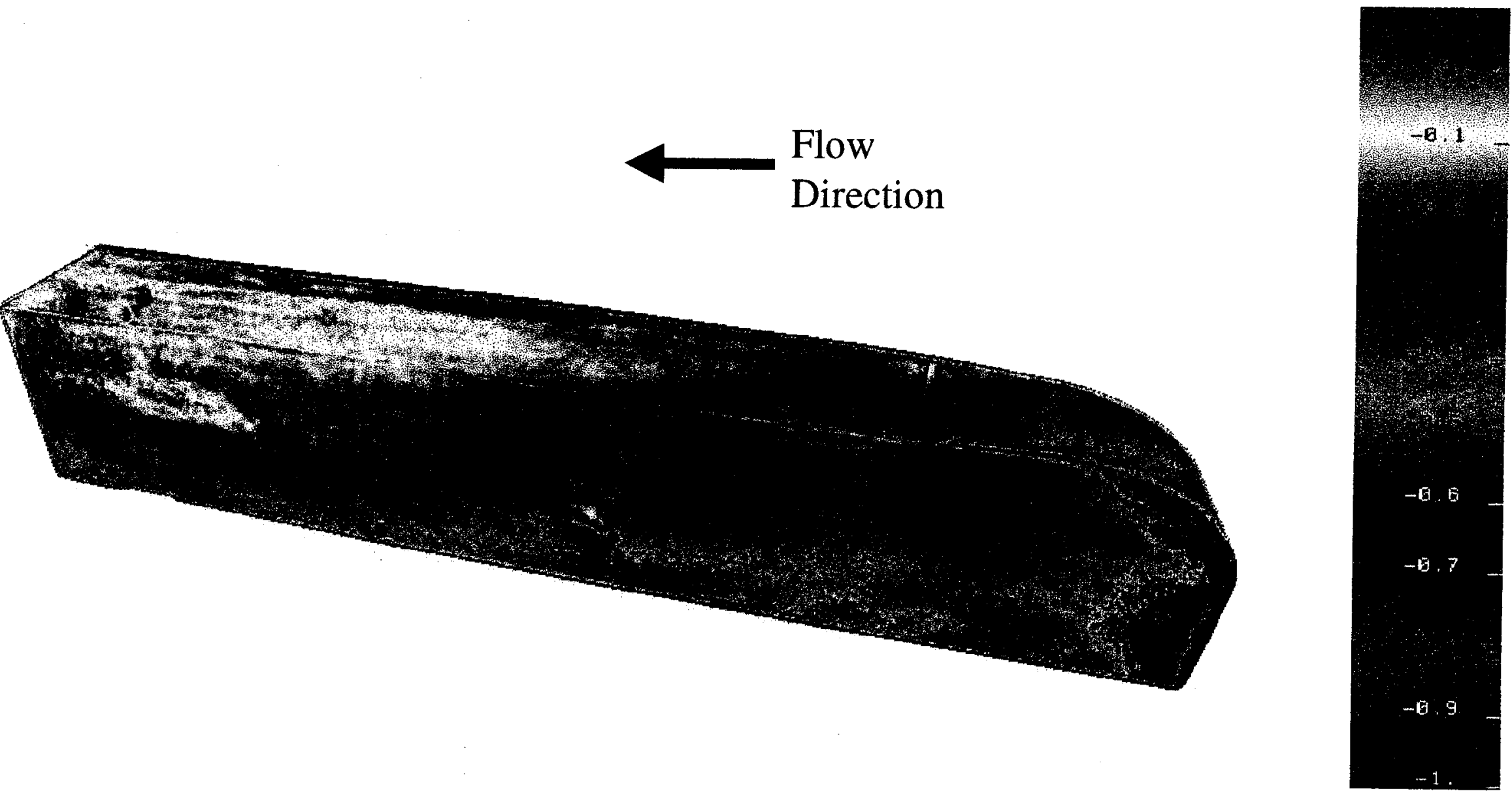


\section{PSP Results on GTS Model in Ames 7x10}

Scatter plot shows tap vs PSP comparison For $\mathrm{Q}=88 \mathrm{psf}$, $\mathrm{Yaw}=10^{\circ}$ case

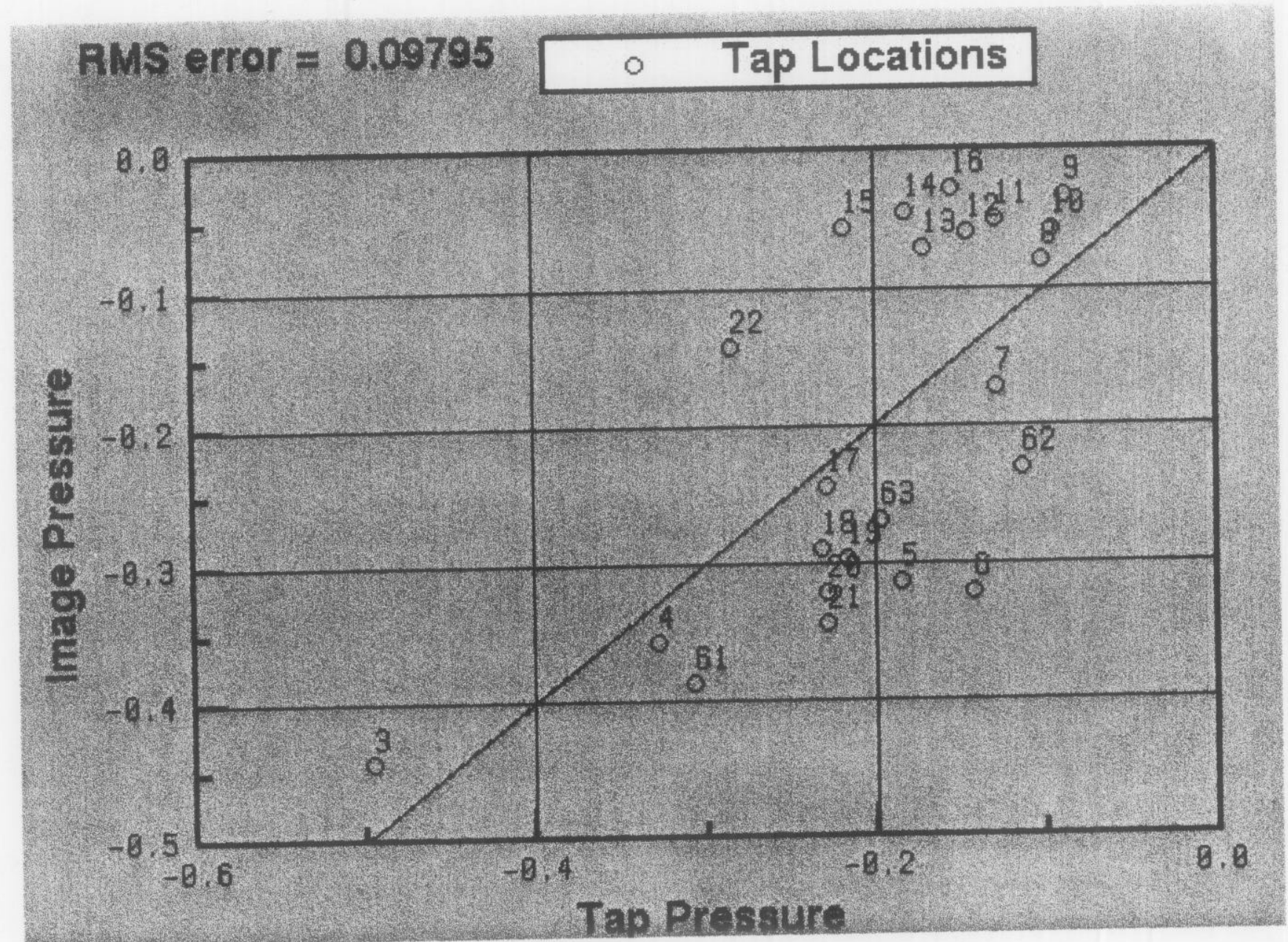




\section{Oil film image}

Top view of trailer at $10^{\circ}$ yaw
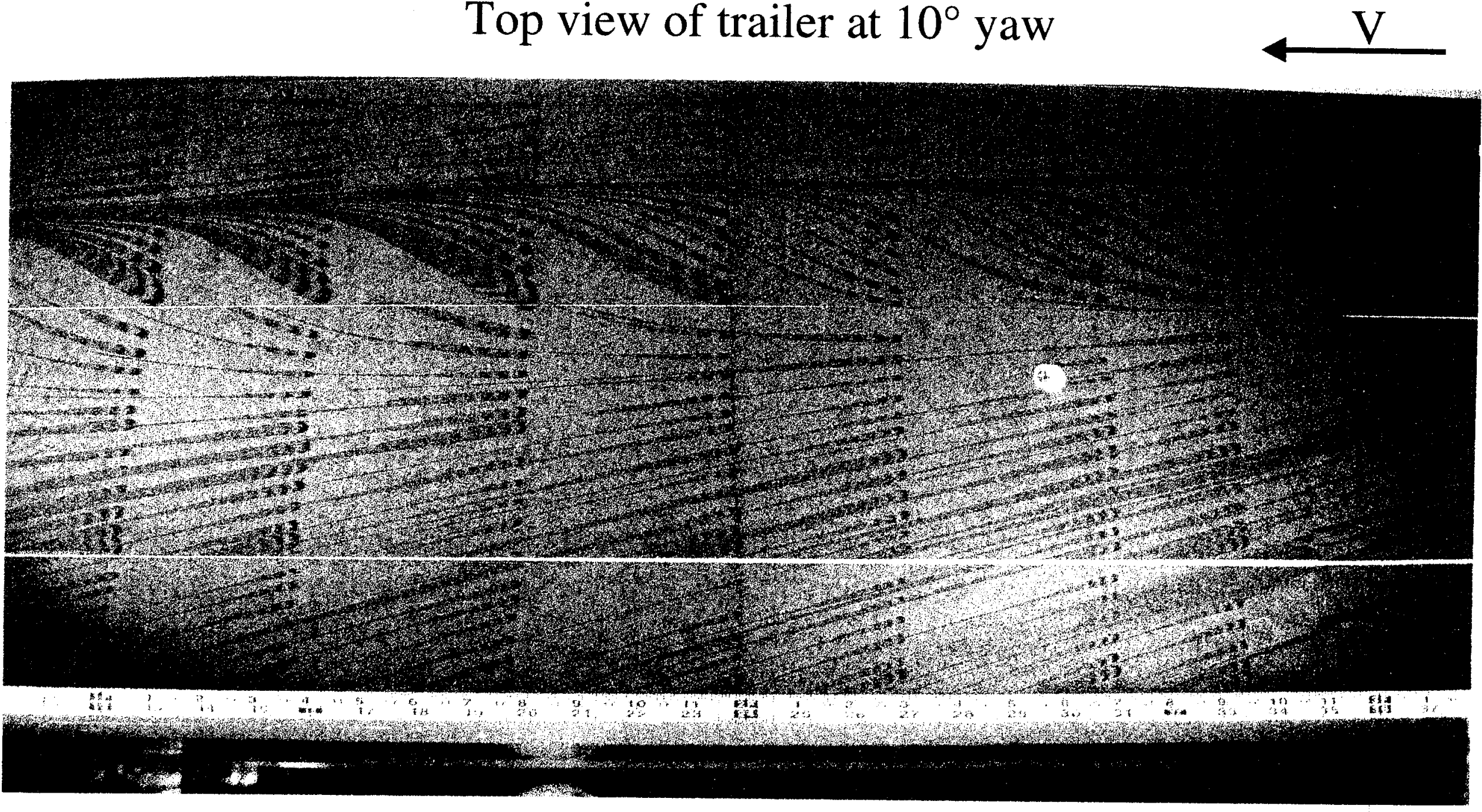

Skin friction proportional to fringe spacing (high under vortex) 
Rear View

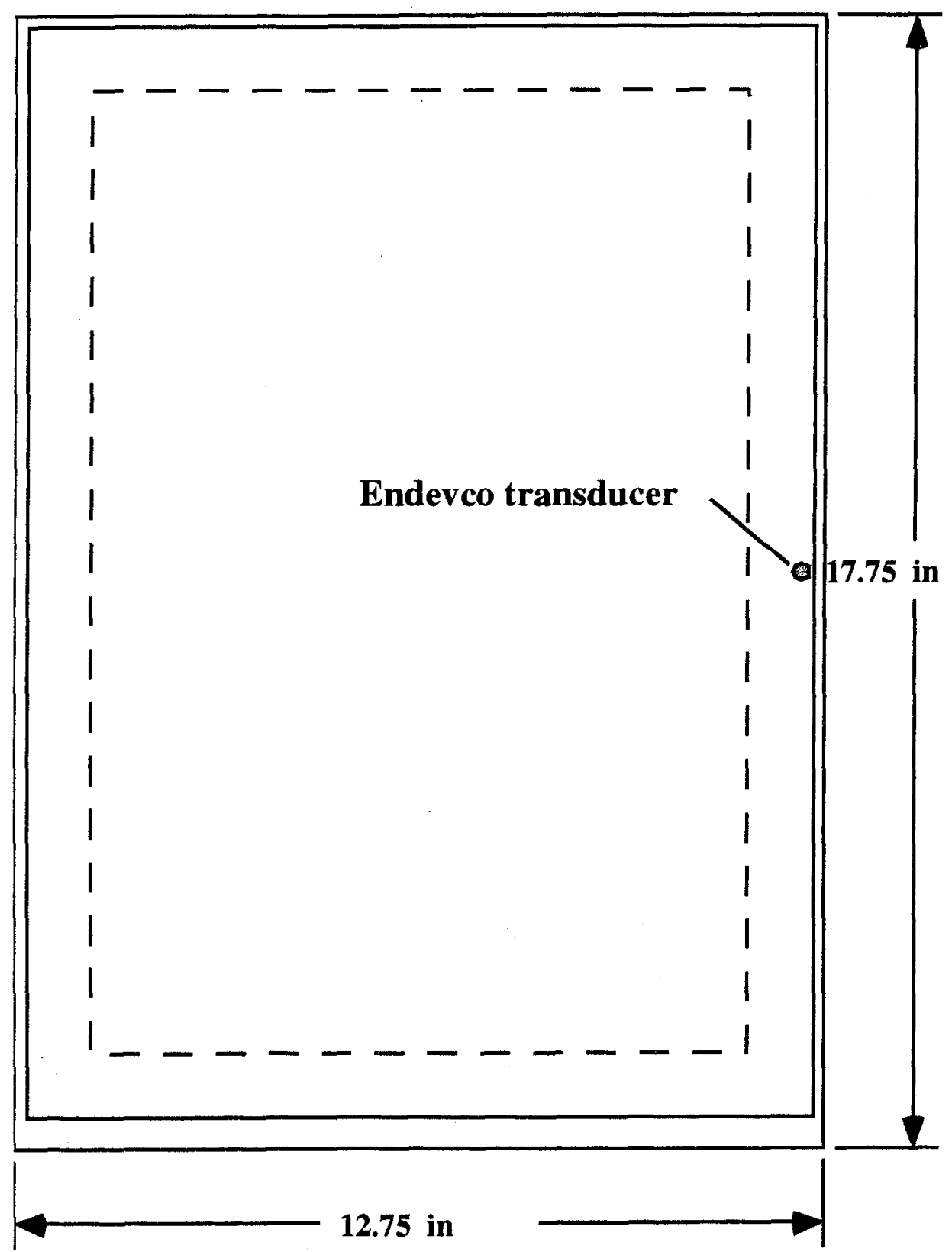

\section{Unsteady Pressure}

Measurements

- 15 psia transducer

- Mid-height on right side of rear door (nose right is positive yaw)

- Center of transducer is 0.25 " from side edge 


\section{Unsteady Pressure Signal $\mathrm{q}=105 \mathrm{psf}, 0^{\circ}$ yaw, baseline}

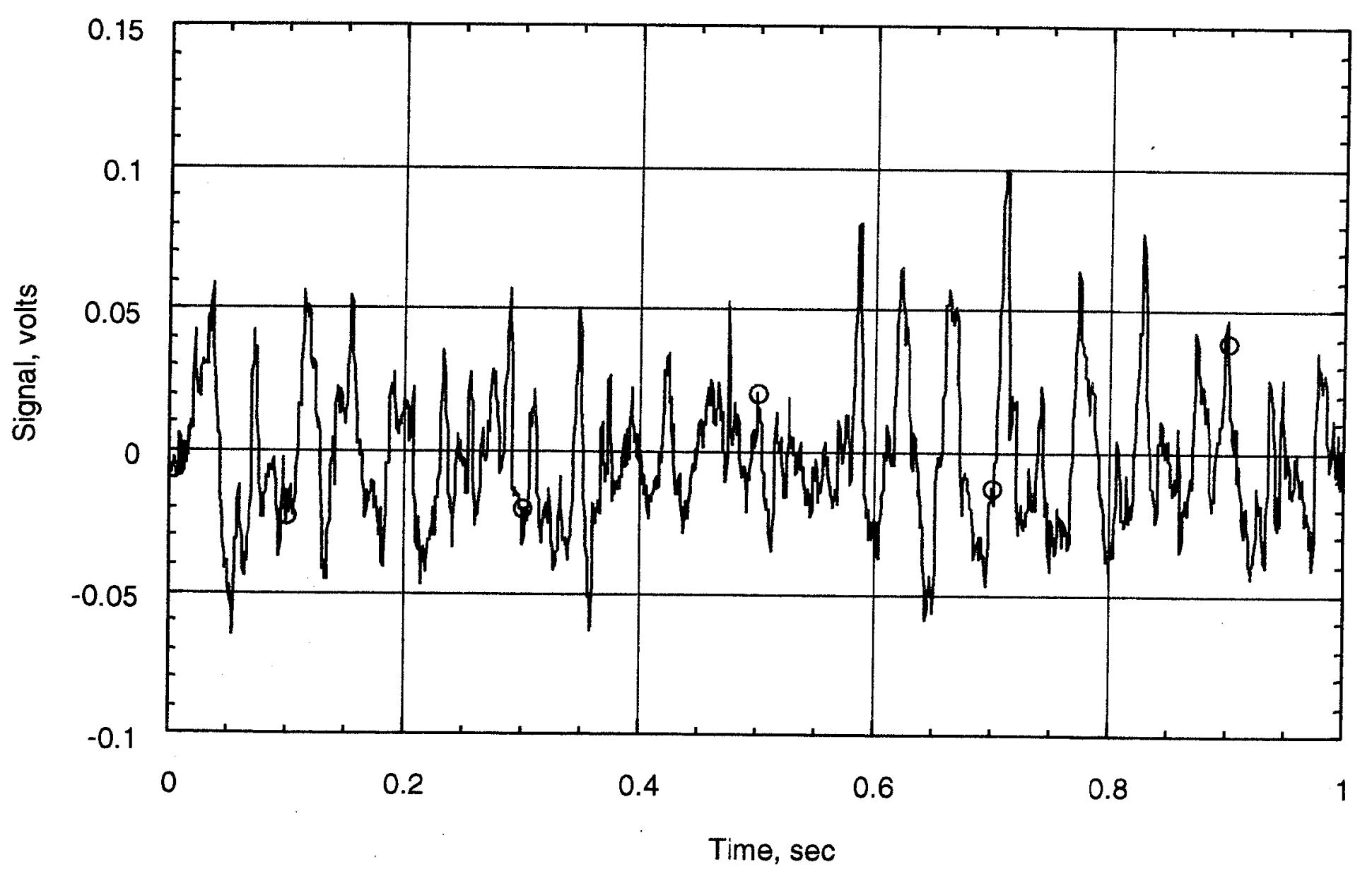




\section{Signal Quality for Unsteady Pressures}

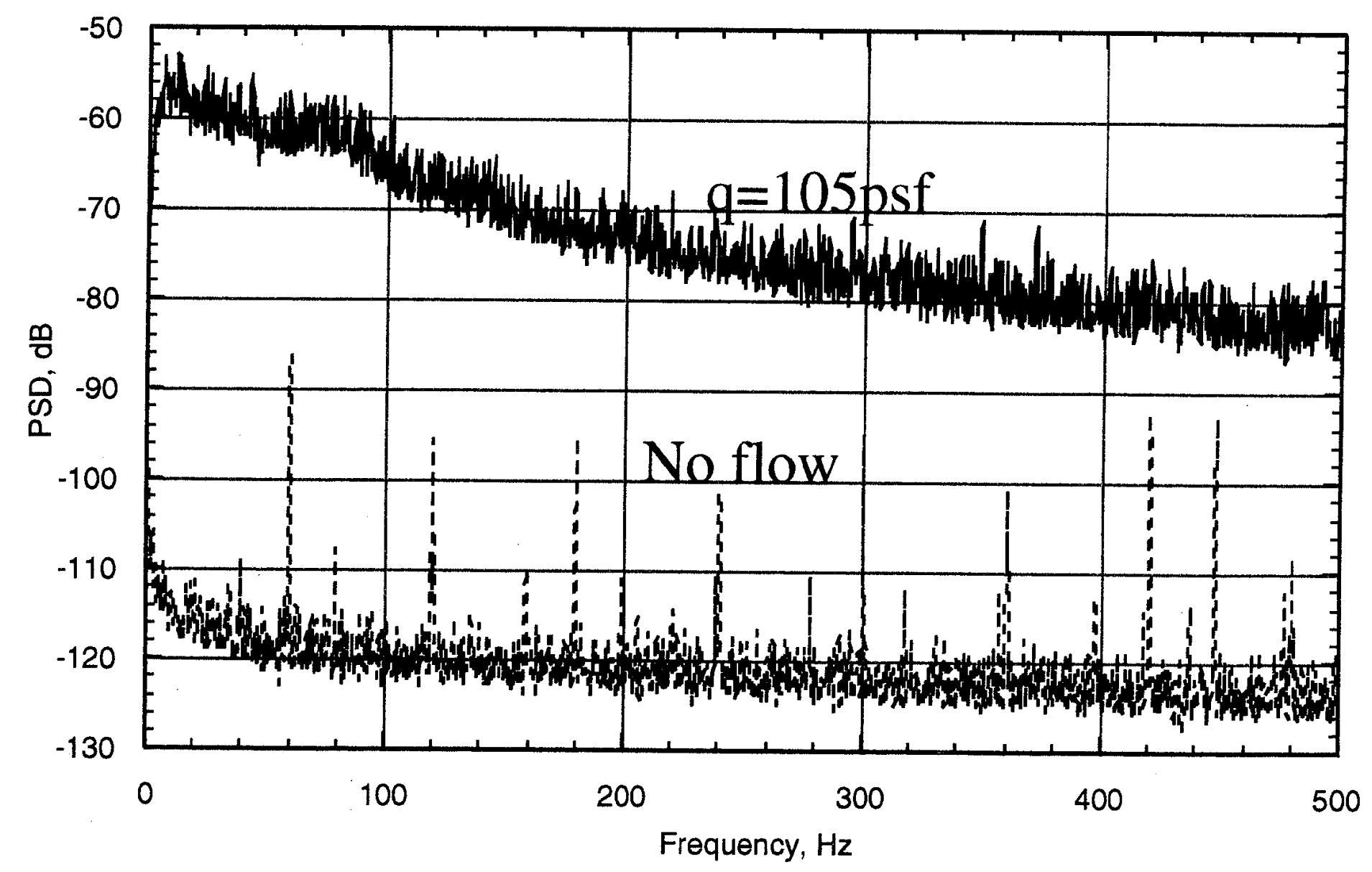




\section{Power Spectrum of Pressure Signal $q=105 \mathrm{psf}, \operatorname{Re}=1.2 \mathrm{M}, 0^{\circ}$ yaw, baseline}

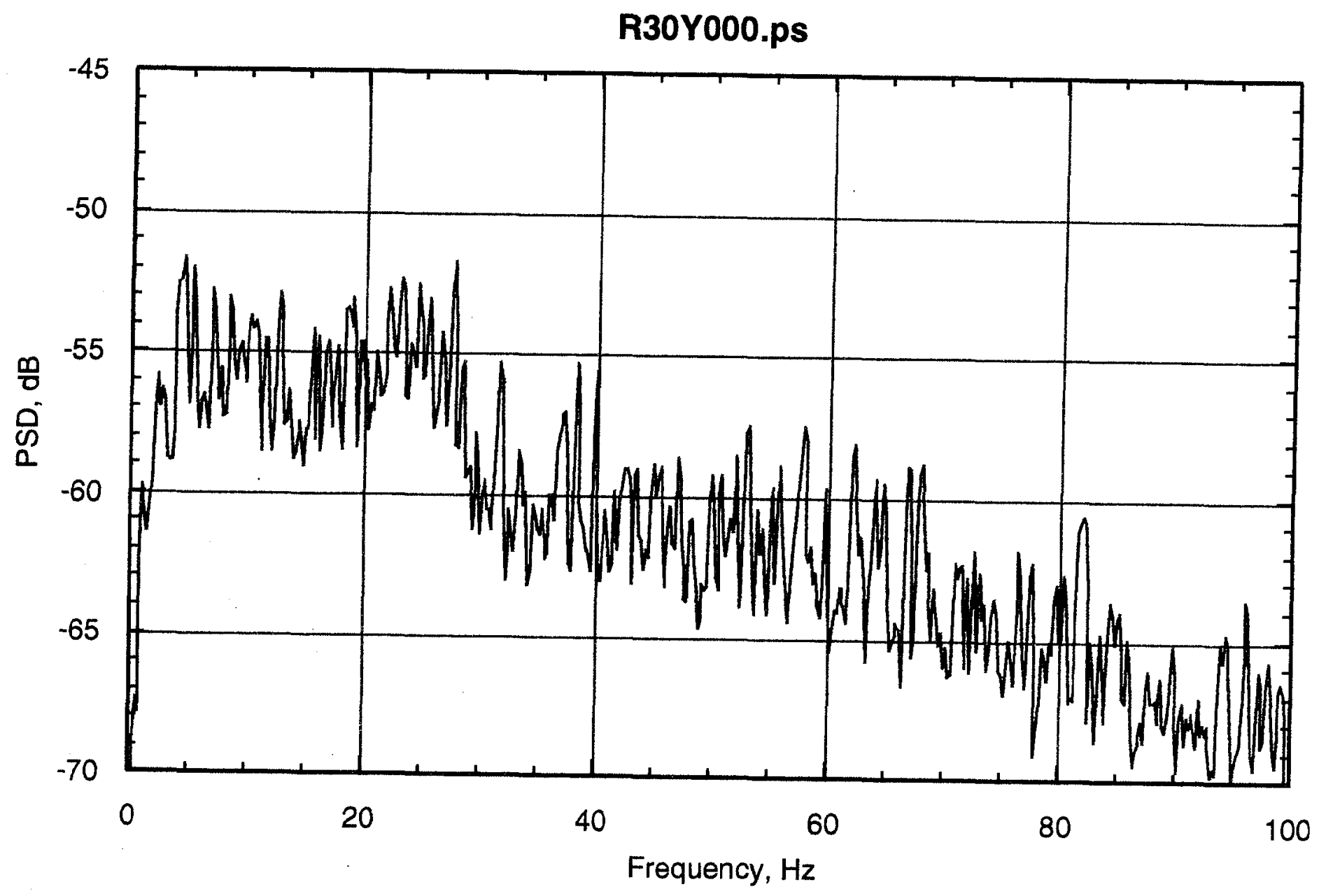




\section{Power Spectrum of Pressure Signal $q=105$ psf $, \operatorname{Re}=1.2 \mathrm{M},-10^{\circ}$ yaw, baseline}

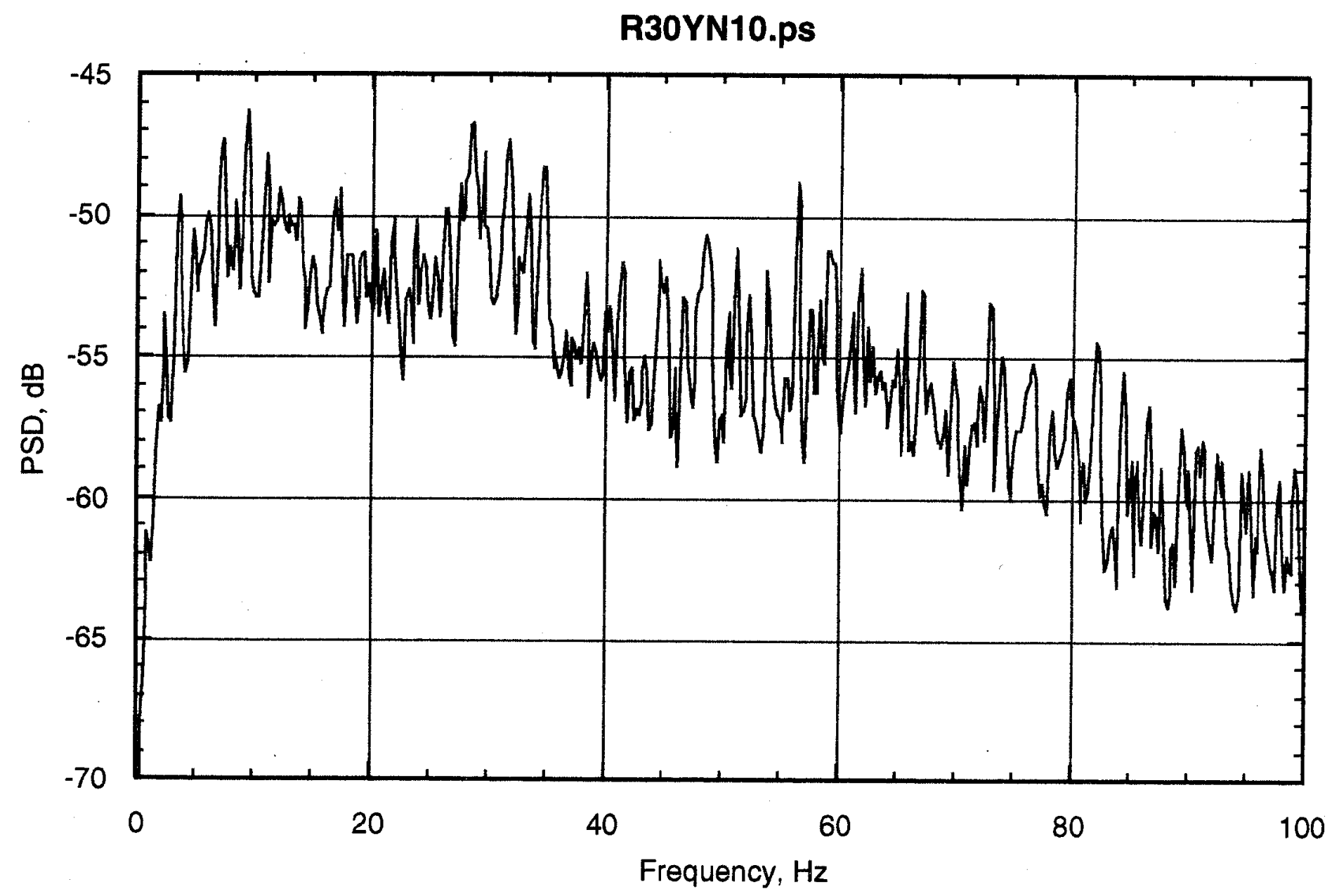




\section{Power Spectrum of Pressure Signal}

$\mathrm{q}=105 \mathrm{psf}, \operatorname{Re}=1.2 \mathrm{M}, 10^{\circ}$ yaw, baseline

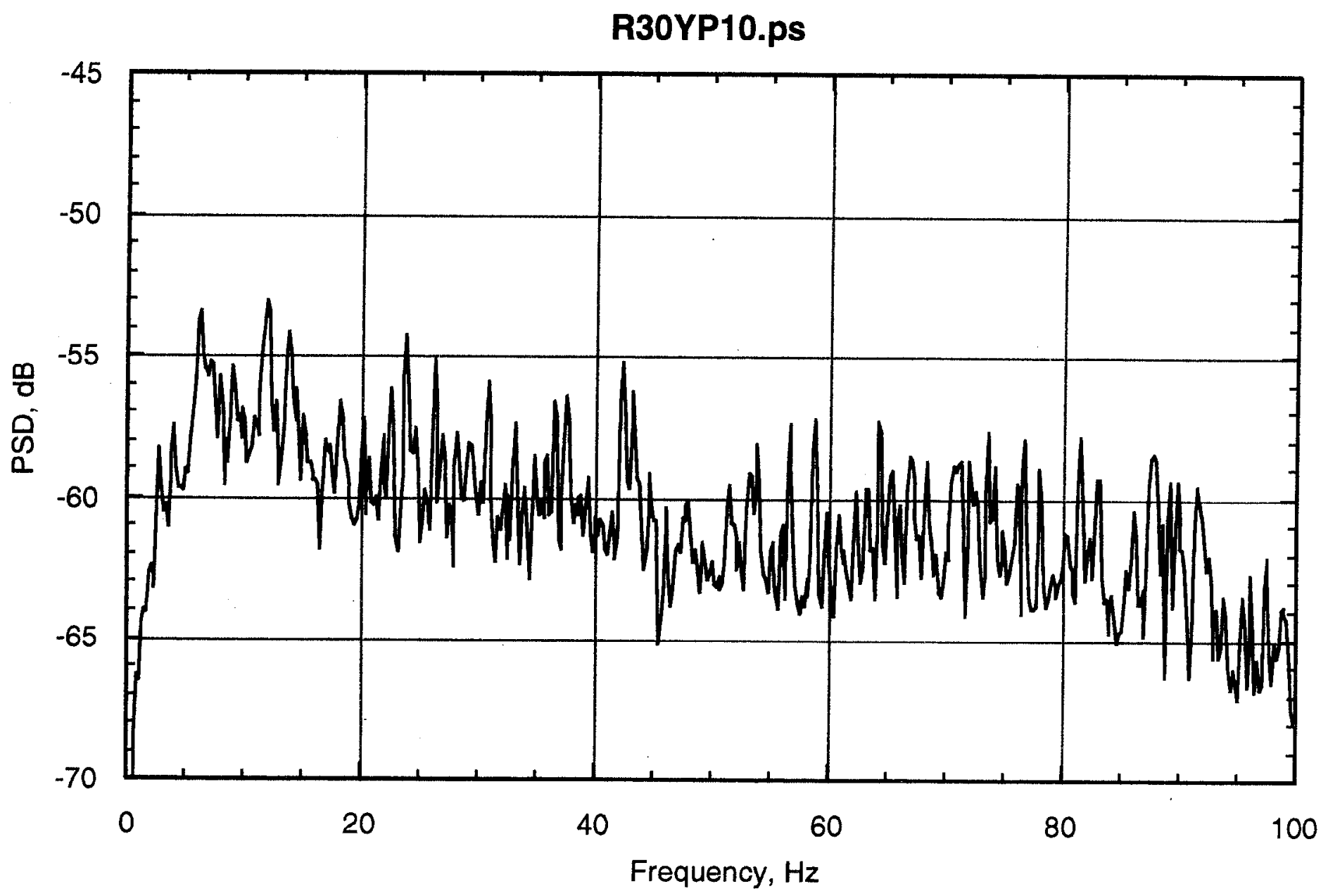




\section{Effect of Boattail Plates on Pressure Spectrum $\mathrm{q}=105 \mathrm{psf}, \operatorname{Re}=1.2 \mathrm{M}, 0^{\circ}$ yaw}

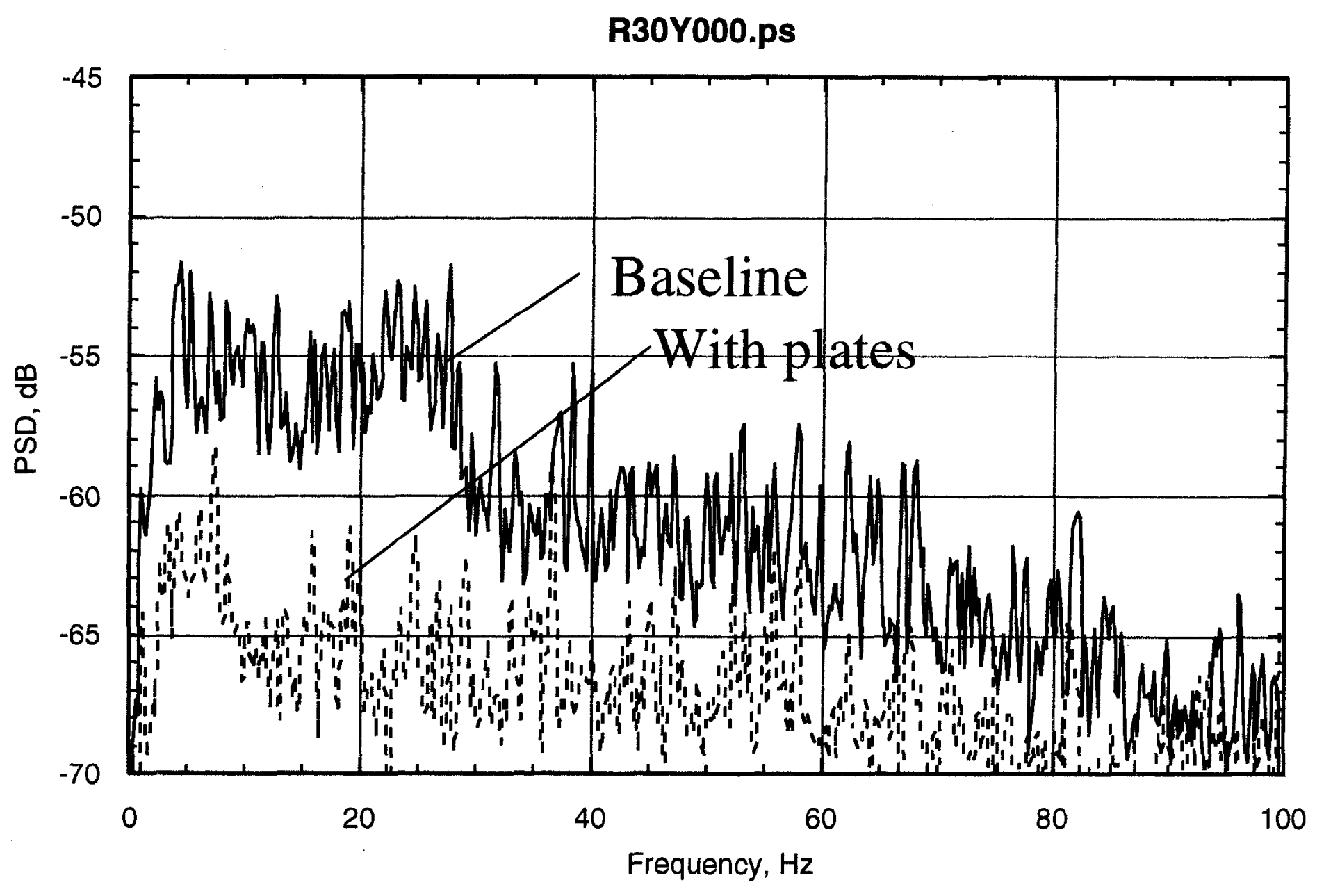




\section{Effect of Boattail Plates on Pressure Spectrum $\mathrm{q}=105 \mathrm{psf}, \operatorname{Re}=1.2 \mathrm{M},-10^{\circ}$ yaw}

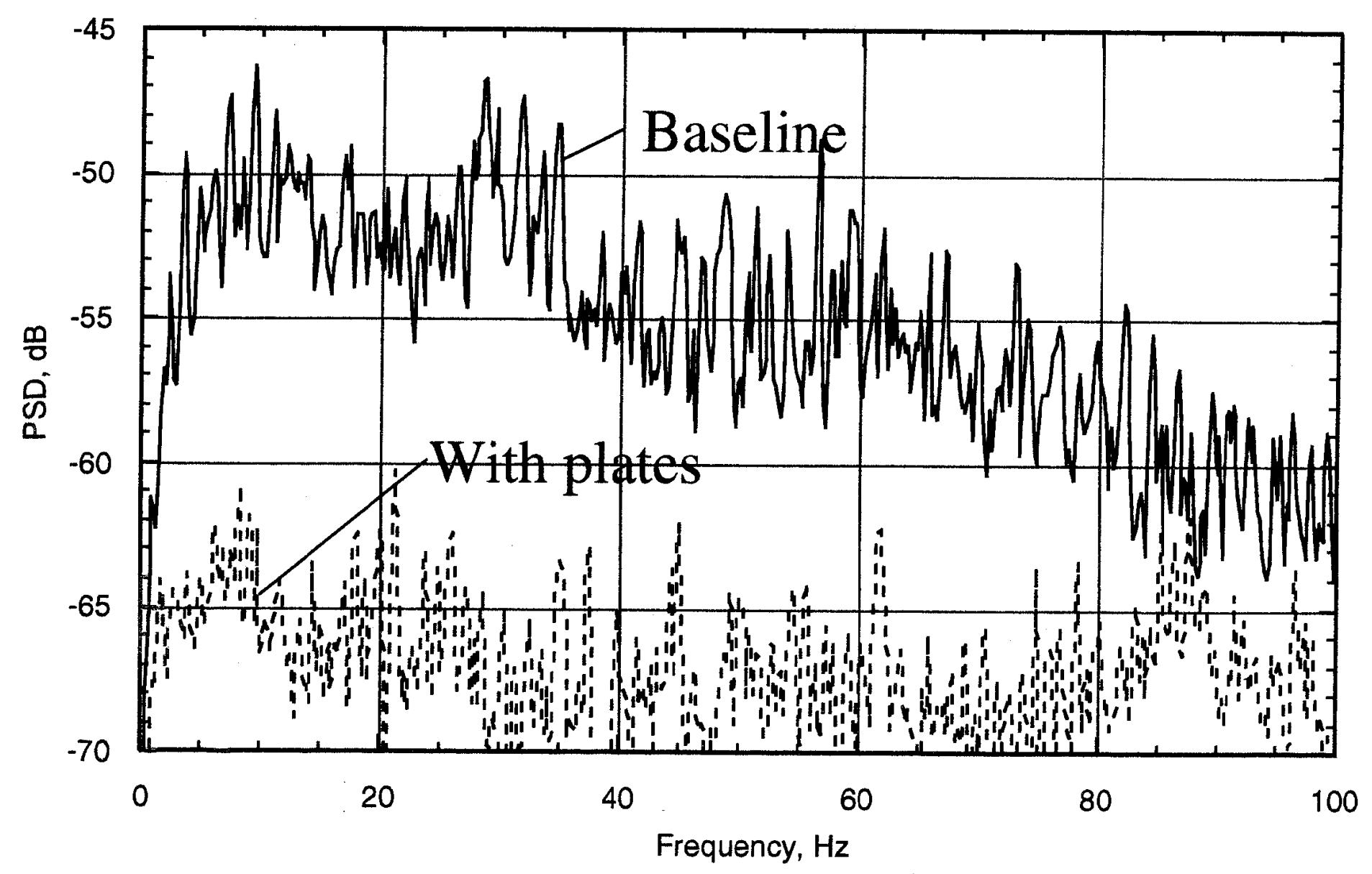




\section{Effect of Boattail Plates on Pressure Spectrum $\mathrm{q}=105 \mathrm{psf}, \operatorname{Re}=1.2 \mathrm{M}, 10^{\circ}$ yaw}

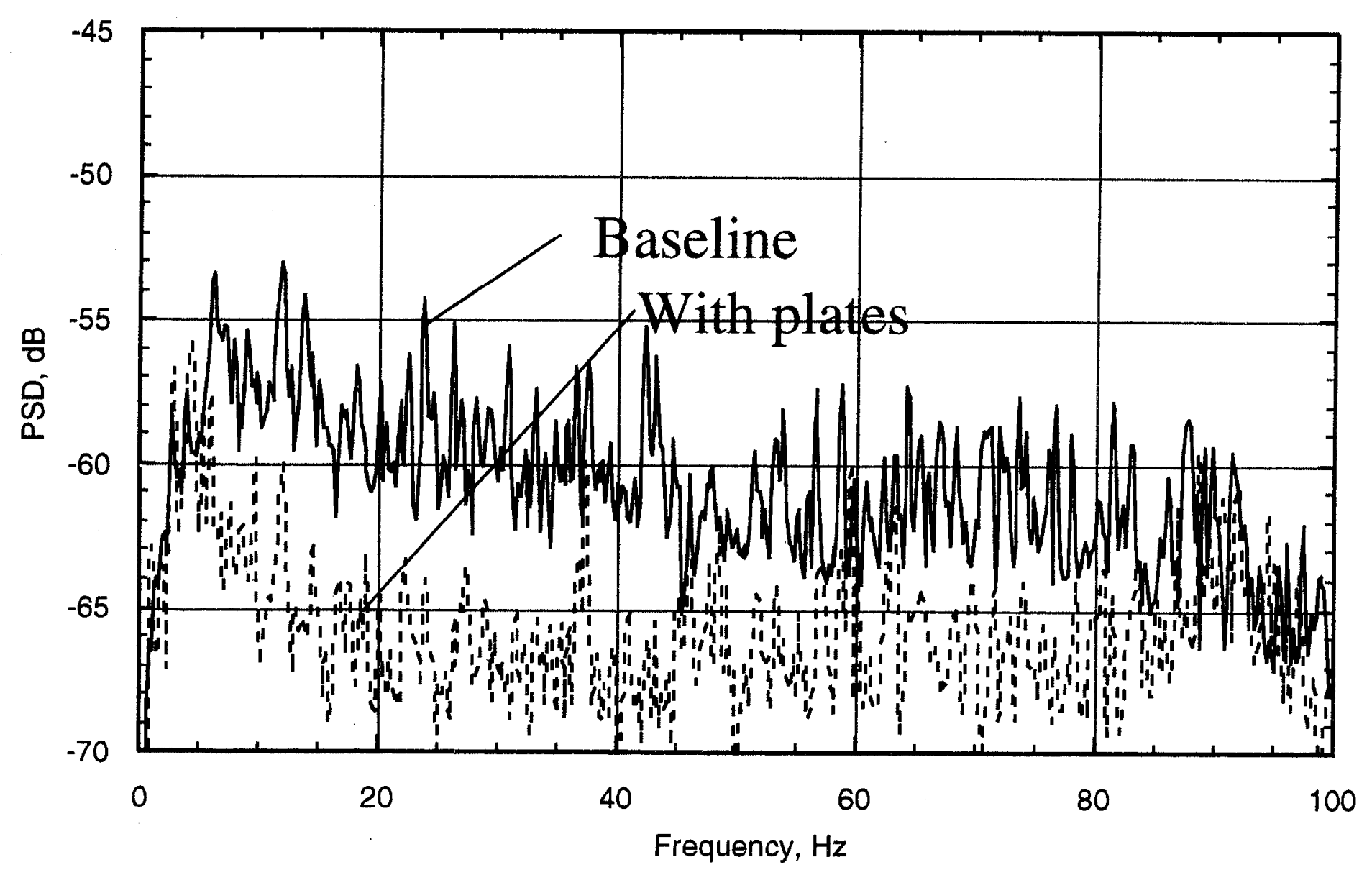




\section{Pressure PSD at Low Speed $q=15 \mathrm{psf}, \operatorname{Re}=450,000,0^{\circ}$ yaw}

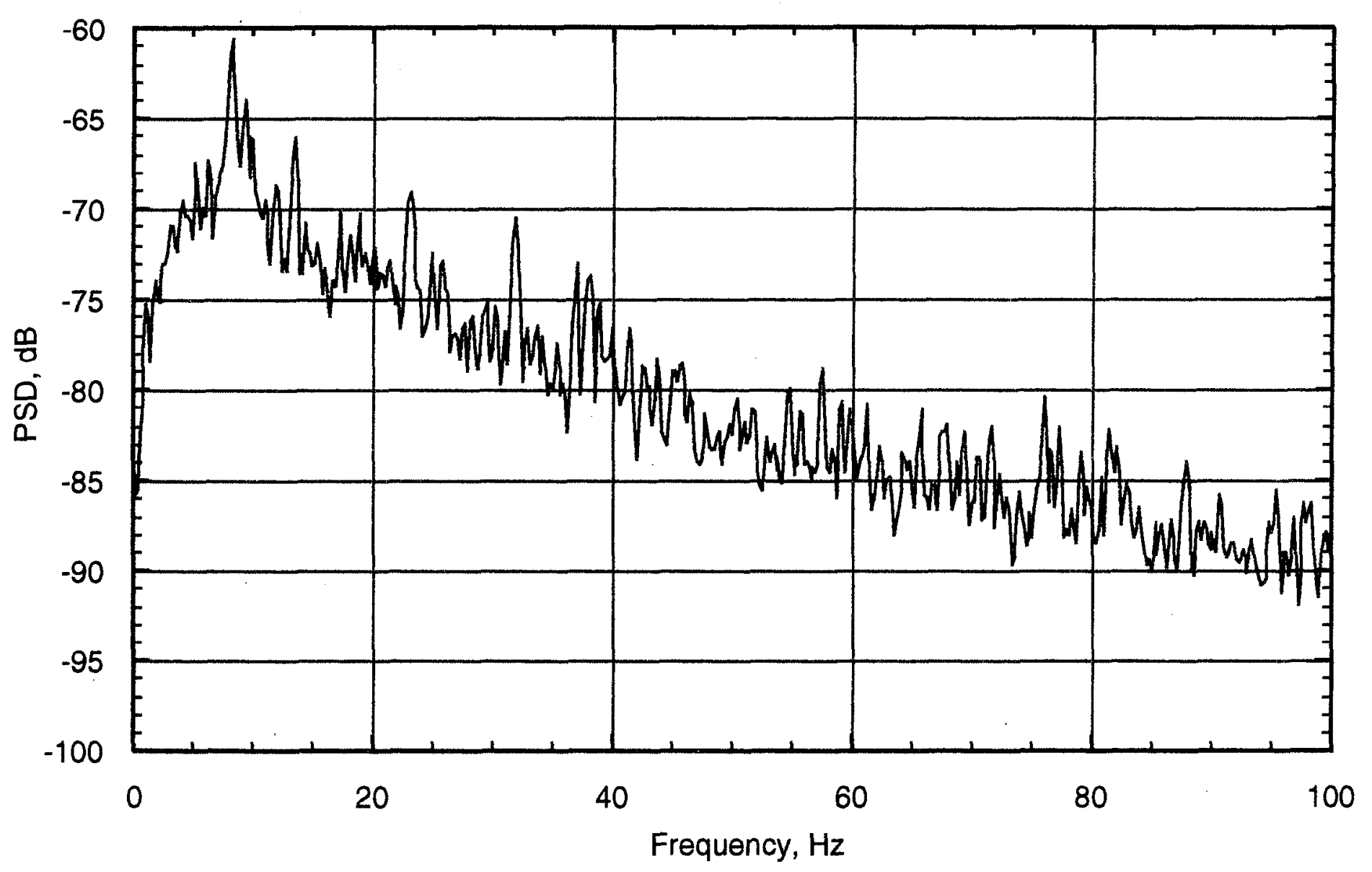


Movie of Wake 


\section{Remaining Work}

- Complete test

- Assemble data report

- Distribute data for validation work

- Data format is an open issue 


\section{Plans for 12' PWT Test}

- Examine Re effects up to full-scale on $1 / 8^{\text {th }}$ scale model

- Determine minimum Re for various aspects of geometry

- Gap

- Cooling flow

- Mirror

- Base-drag reduction

- ?

- CFD validation data at range of $\mathrm{Re}$ 


\section{Aerodynamics of Heavy Vehicles}

Fred Browand Mustapha Hammache Staff

Glen Landreth David Sandler

Patricia Wall Students

\section{Aerospace and Mechanical Engineering University of Southern California}

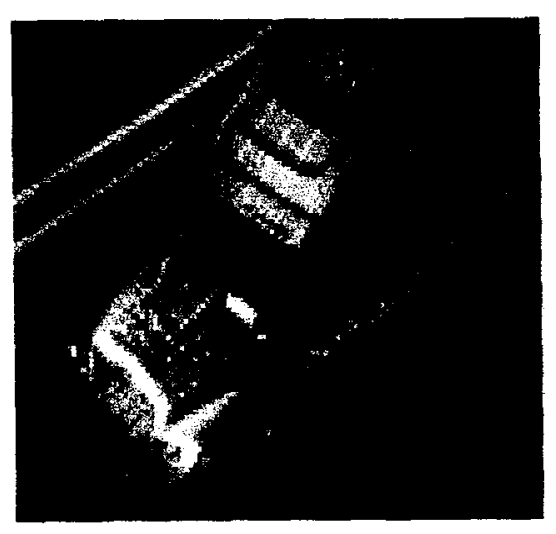

LLNL
March 11, 1999 


\section{Introduction}

- Brief discussion of several relevant publications

- Progress in the laboratory

- Computation

- Recent DPIV measurements 
- "Detached-eddy simulation of an airfoil at high angle of attack", M. Shur, P.R. Spalart, M. Strelets, A. Travin, Proceedings of the $1^{\text {st }}$ AFOSR Conference on DNS/LES, Ruston, LA, August 1997

- "Comments on the feasibility of LES for wings, and on a hybrid RANS/LES approach", P.R. Spalart, W-H. Jou, M. Strelets, S.R. Allmaras

- 200,000 grid points, fully 3-D calculation

- RANS formalism for attached boundary layers

- LES sufficiently far away from wall

- Switches between the two automatically, (this is DES)

- Captures bulk of unsteady flow behavior 
- "The effect of front-edge rounding and rear-edge shaping on the aerodynamic drag of bluff vehicles in ground proximity", K.R. Cooper, SAE Technical Paper 850288, 1985

- Preventing separation at relatively sharp front edges can have a dramatic effect on the drag of a bluff body 
- "Flight-determined subsonic lift and drag characteristics of seven liftingbody and wing-body reentry vehicle configurations with truncated bases", E.J. Saltzman, K.C. Wang, K.W. Iliff, AIAA 99-0383, 1999

- "A reassessment of heavy-duty truck aerodynamic design features \& priorities", E.J. Saltzman, R.R. Meyer, Jr., Preliminary Report, NASA Dryden Flight Research Center, 1998

- A relationship exists between forebody drag and base pressure coefficient for many bluff-base aerodynamic shapes. This may include heavy trucks.

- If the form of the relationship is postulated, the minimum drag configuration can be determined 


\section{Progress in the laboratory}

- Work area being remodeled (University pays $\$ 25,000$ )

- Yaw turntable will be operational in April

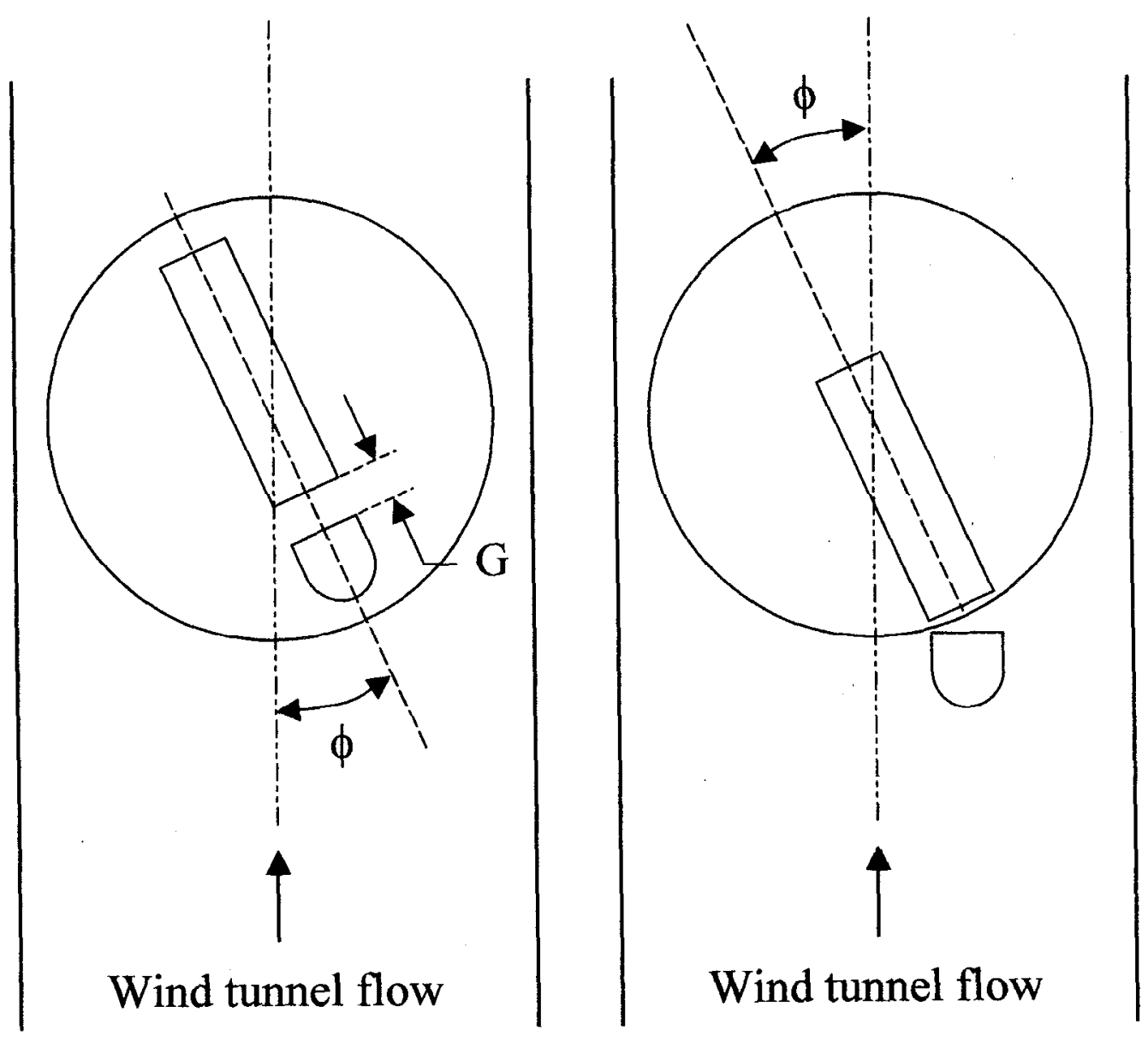

USC March 1999 Contractors meeting 
- SNL Truck Model has been fabricated and operated in the wind tunnel
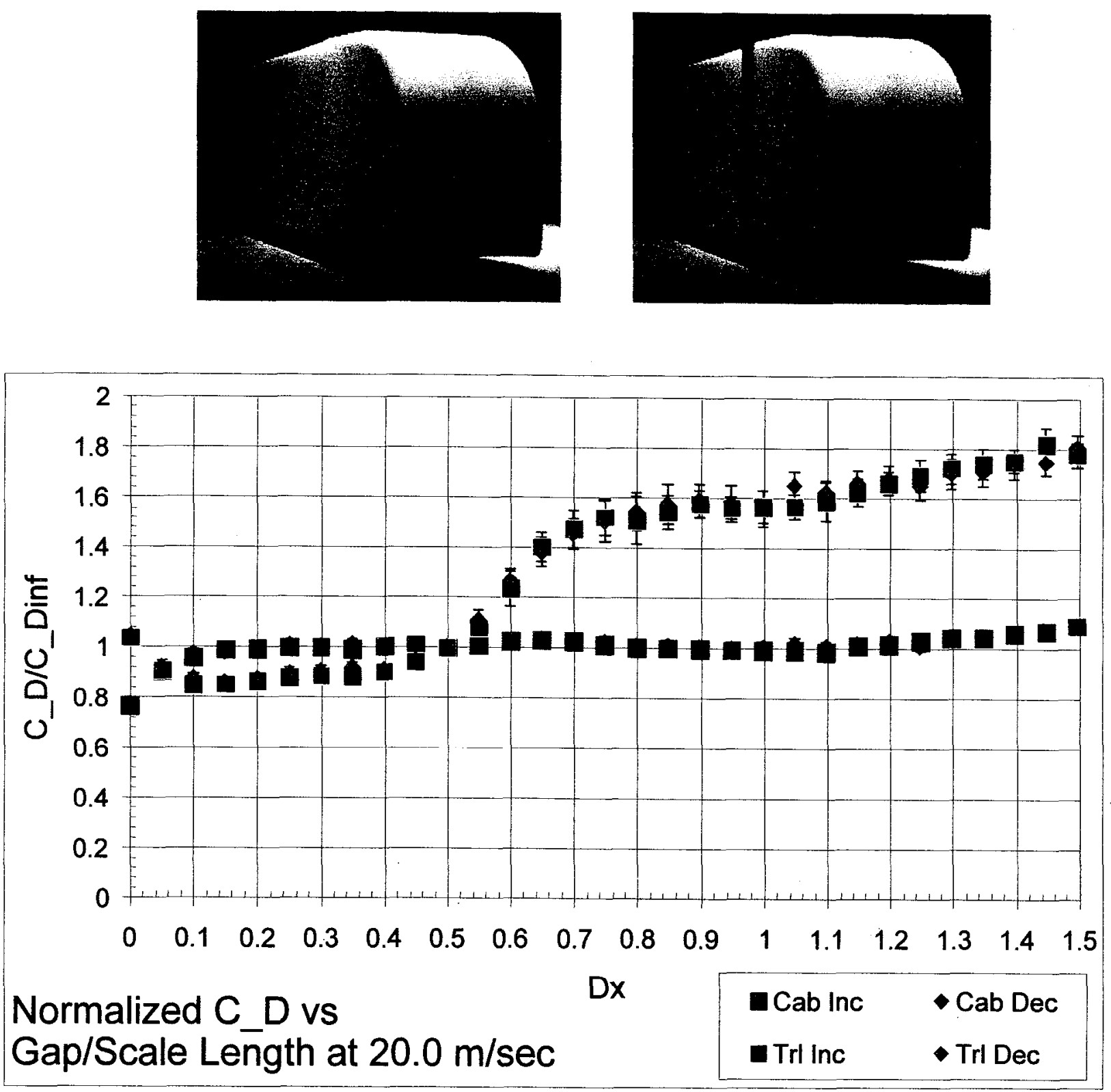

USC March 1999 Contractors meeting 

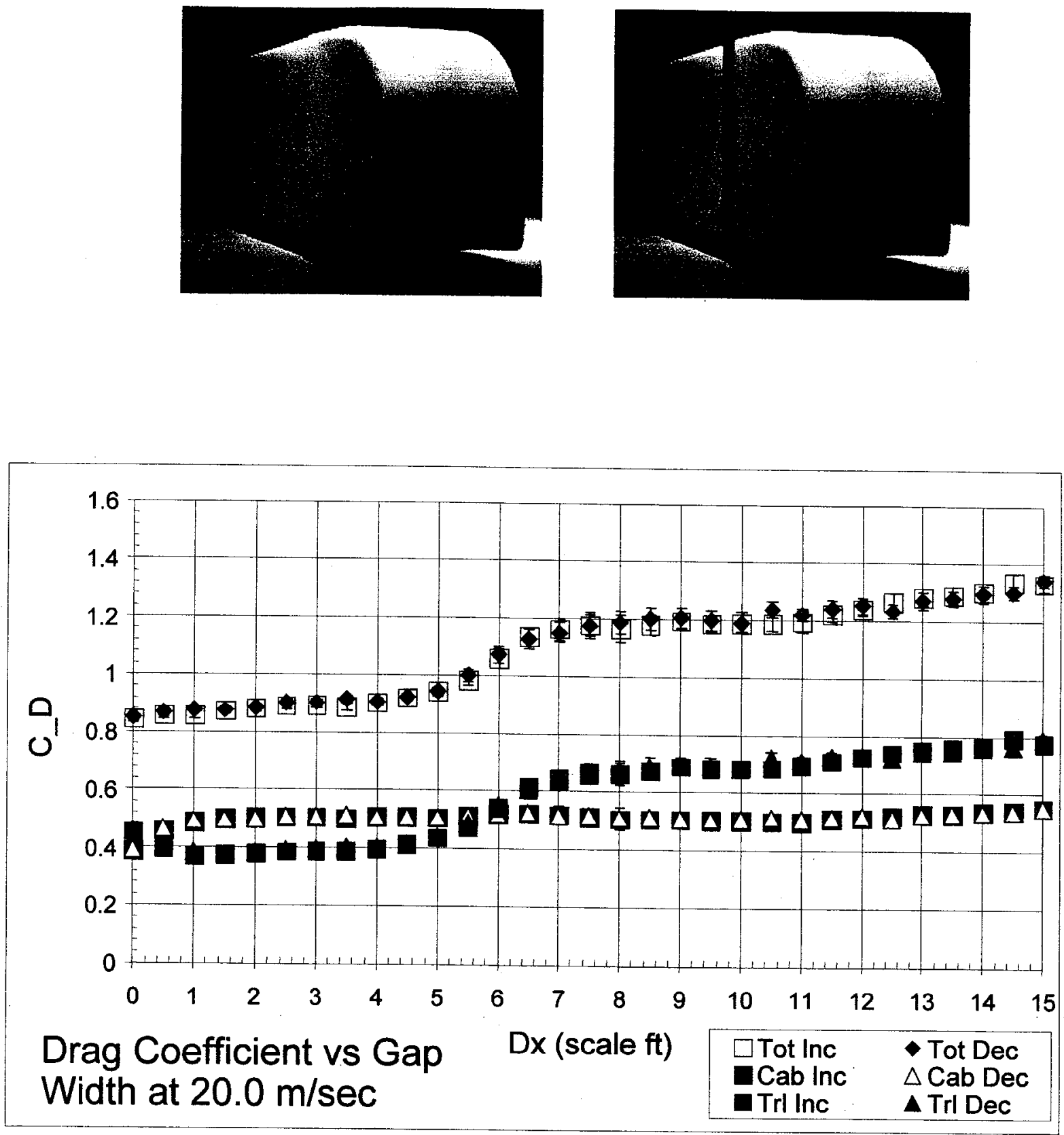

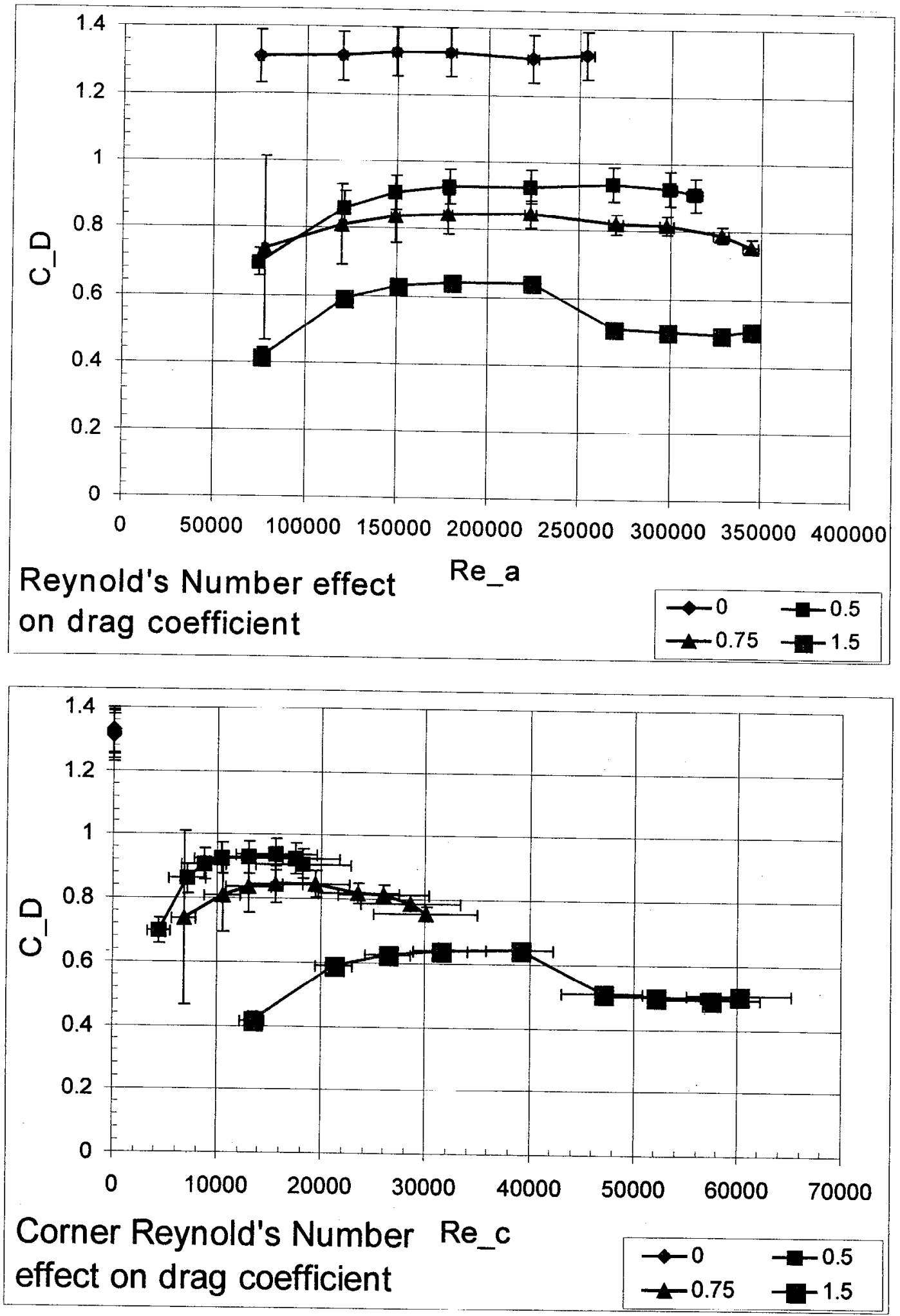

USC March 1999 Contractors meeting 


\section{Computations}

- Panel code calculations PMARC

- Visiting Scientist:

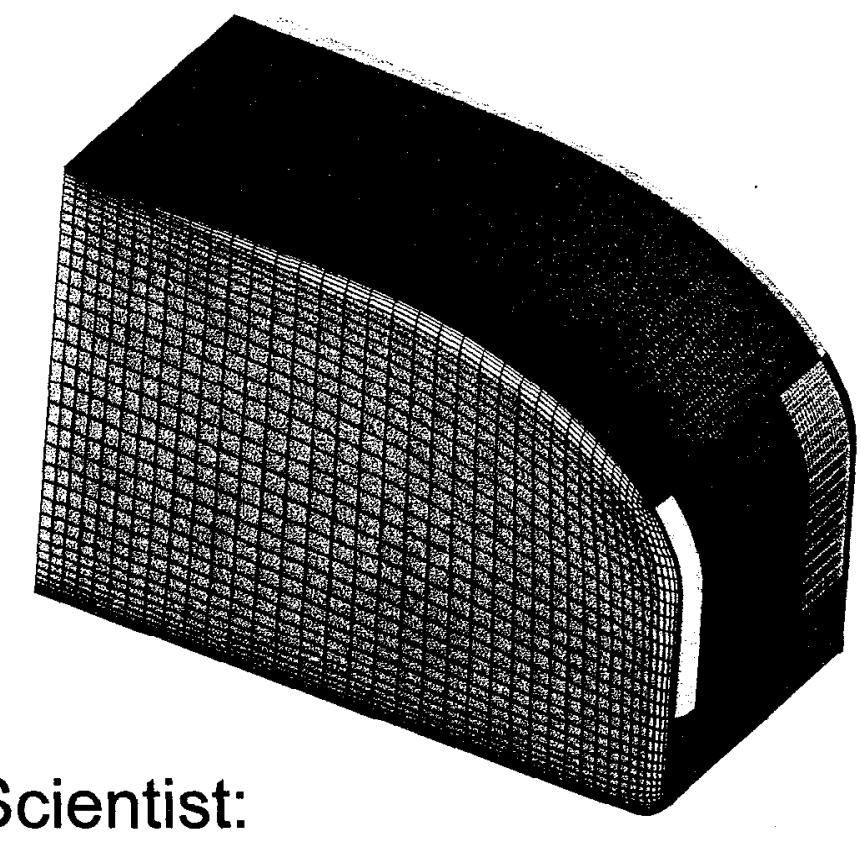

Dr. Dieter Schwamborn

Head, CFD Group

Institute for Fluid Mechanics

DLR, Gottingen (Germany)

- DES:Smooth cab-trailer (with gap) SNL Model (with cab-trailer gap) 


\section{Topics of
Discussion}

- Description of flow facilities

- Description of DPIV capabilities

- Maximizing accuracy

- Maximizing spatial resolution

- Treating solid boundaries

- Experiments

- Vehicle platooning

- SNL truck model with gap

- The NASA-Ames PIV data at low speed 


\section{Flow facilities}

- Dryden wind tunnel

- Top speed $44 \mathrm{~m} / \mathrm{s}$ (100mph)

- Test section $1.3 \mathrm{~m} \times 1.3 \mathrm{~m}$ (53"x53")

- Nissan/Linac wind tunnel

- Moving ground belt

- Top speed $90 \mathrm{~m} / \mathrm{s}$

- Test section $0.9 \mathrm{~m} \times 1.1 \mathrm{~m}(36$ "x42") 


\section{DPIV capabilities}

- Two-dimensional PIV based on crosscorrelation technique

- Dual channel CCD camera

- 1 million pixels

- 15 images pairs/sec

- Dual Nd-Yag laser

- $160 \mathrm{~mJ} / \mathrm{pulse}$

- $10 \mathrm{~Hz}$

- Option for variable resolution

- wide flow field

- maximum spatial resolution

- CIV software tested for reliability and speed

- No-slip condition imposed at the walls

- Special treatment of the data close to the walls 
Adaptive resolution
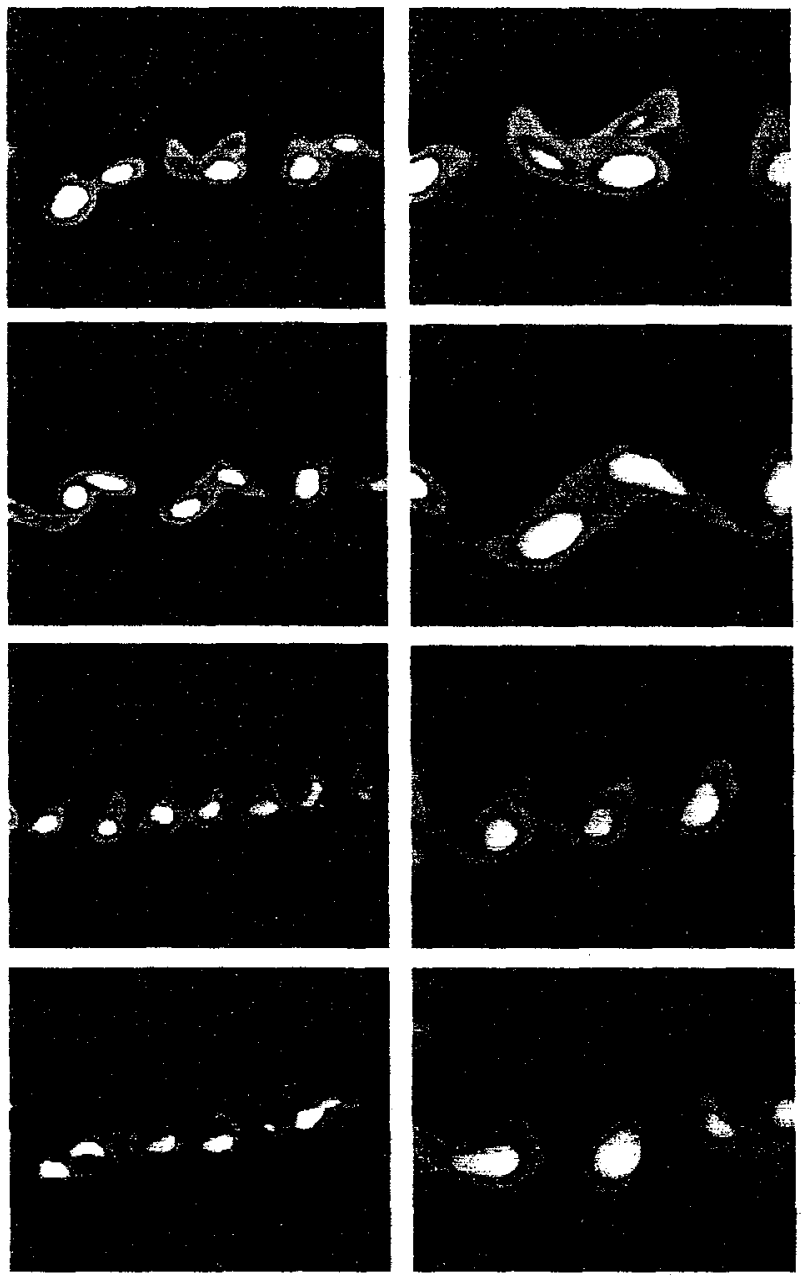

from: G.R. Spedding, "The evolution of initially turbulent bluff-body wakes at high internal Froude number" JFM 337 (1997) 


\section{Vans in back-to-back configuration}

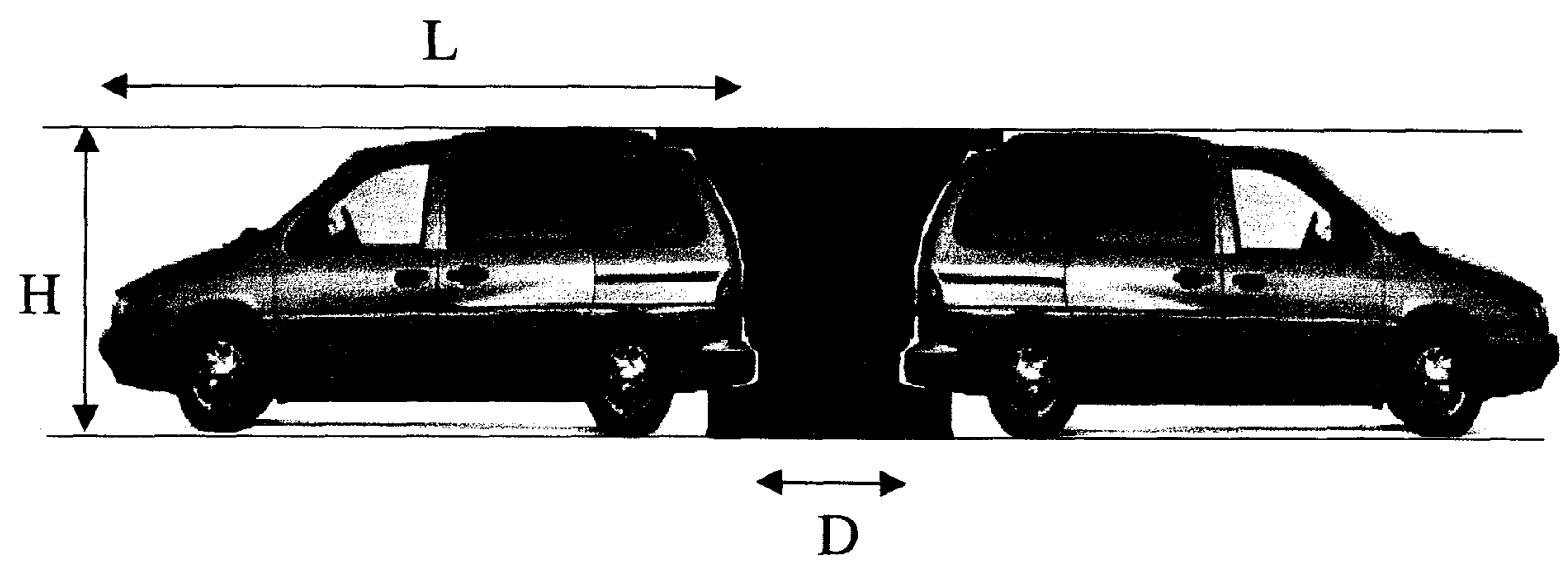

\begin{tabular}{|l||l|}
\hline Van dimensions & $\mathrm{L}=25 ", \mathrm{H}=8 "$ \\
\hline Free-stream velocity & $25 \mathrm{~m} / \mathrm{s}$ \\
\hline Horizontal planes & $28 \%, 46 \%, 65 \%, 84 \%$ of $\mathrm{H}$ \\
\hline Vertical plane & Centerline \\
\hline Spacing & $\mathrm{D} / \mathrm{L}=23 \%$ and $6 \%$ \\
\hline \hline & \\
\hline \hline Seeding & One-micron droplets \\
\hline Illumination & Two Nd-Yag lasers, 160 mJoule/pulse \\
\hline Acquisition rate & $10 \mathrm{~Hz}$ \\
\hline Camera & $1000 \times 1000$ pixels, dual channel CCD \\
\hline Image processing & $\mathrm{CIV} / \mathrm{USC}$ \\
\hline
\end{tabular}


Velocity field for $D / L=23 \%$

Horizontal plane at $\mathrm{Z} / \mathrm{H}=0.28$

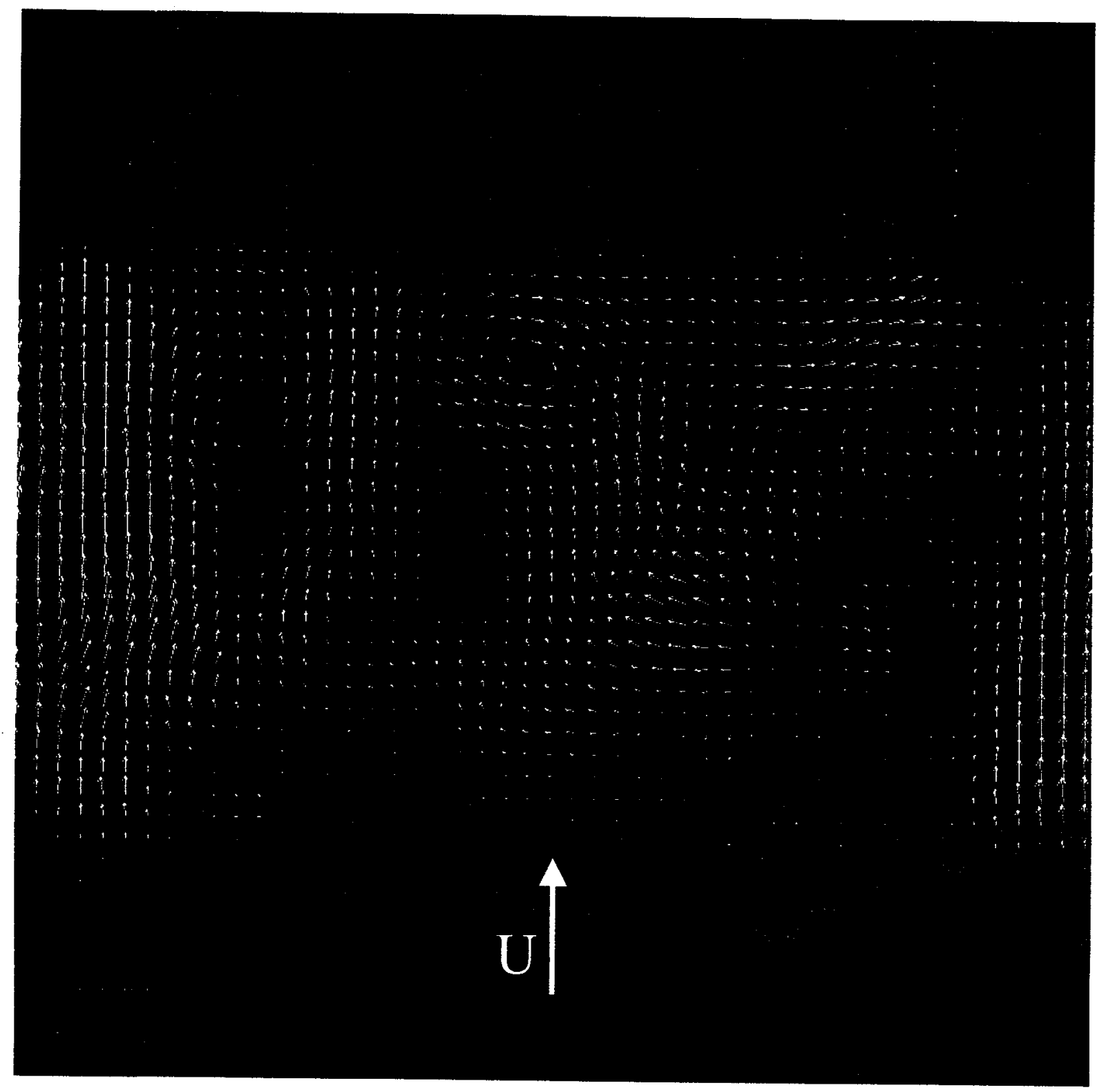

USC March 1999 Contractors meeting 
Velocity field for $D / L=23 \%$

Horizontal plane at $\mathrm{Z} / \mathrm{H}=0.46$

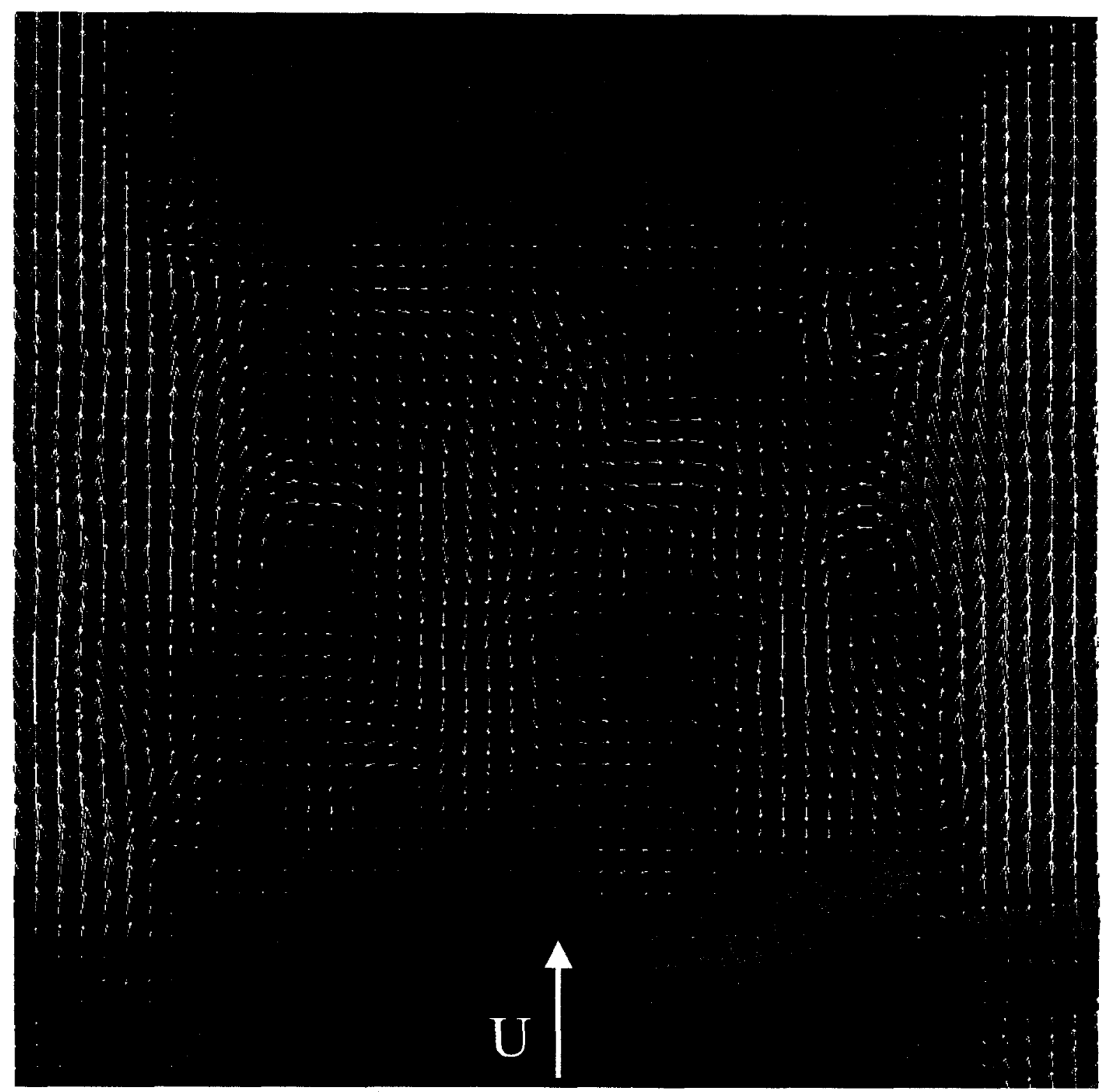

USC March 1999 Contractors meeting 
Velocity field for $D / L=23 \%$ Horizontal plane at $\mathrm{Z} / \mathrm{H}=0.65$

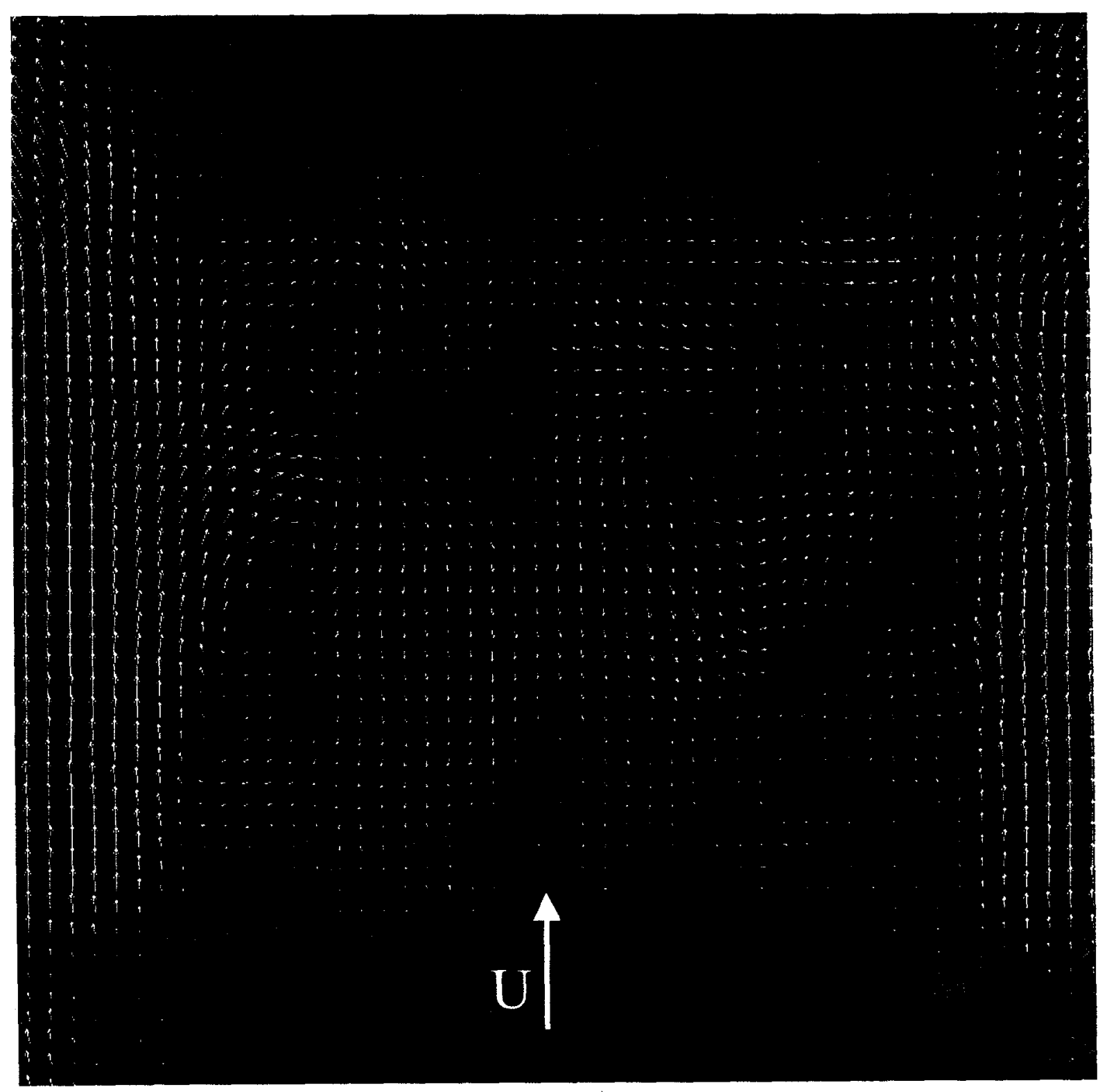

USC March 1999 Contractors meeting 


\section{Velocity field for $D / L=23 \%$ Horizontal plane at $\mathrm{Z} / \mathrm{H}=0.84$}

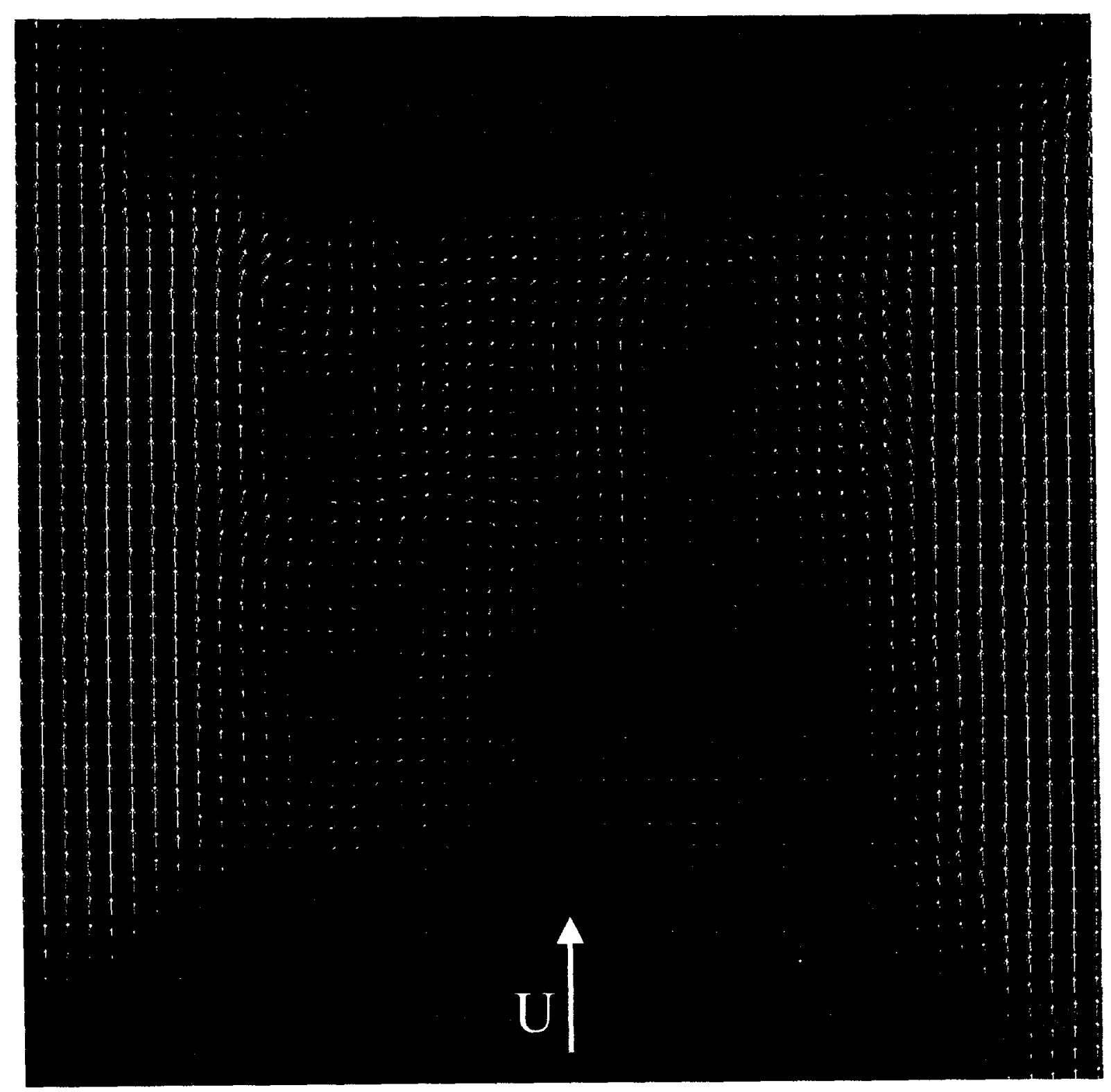


Velocity field for $D / L=6 \%$

Horizontal plane at $\mathrm{Z} / \mathrm{H}=0.46$

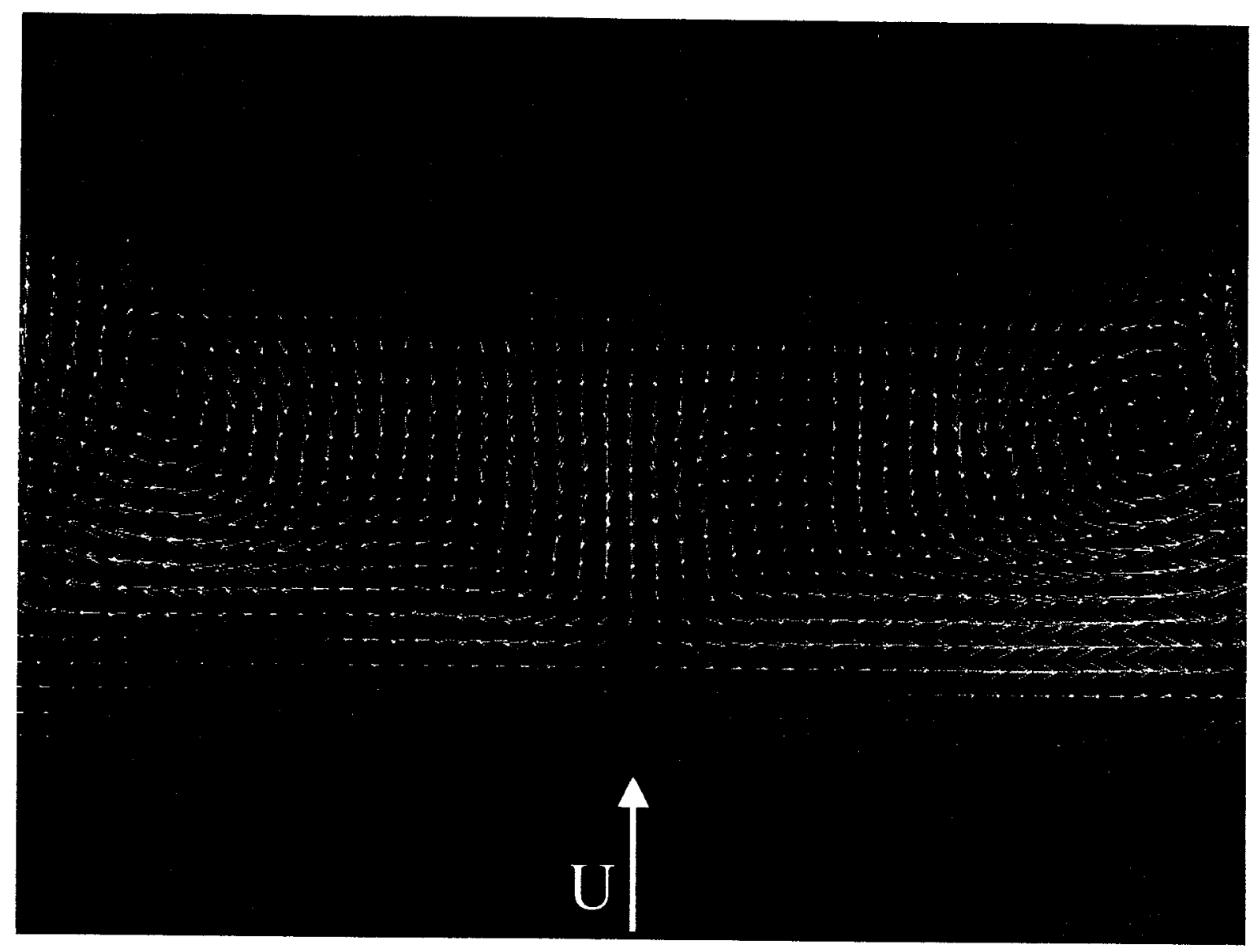

USC March 1999 Contractors meeting 
Velocity field for $D / L=6 \%$

Horizontal plane at $\mathrm{Z} / \mathrm{H}=0.65$

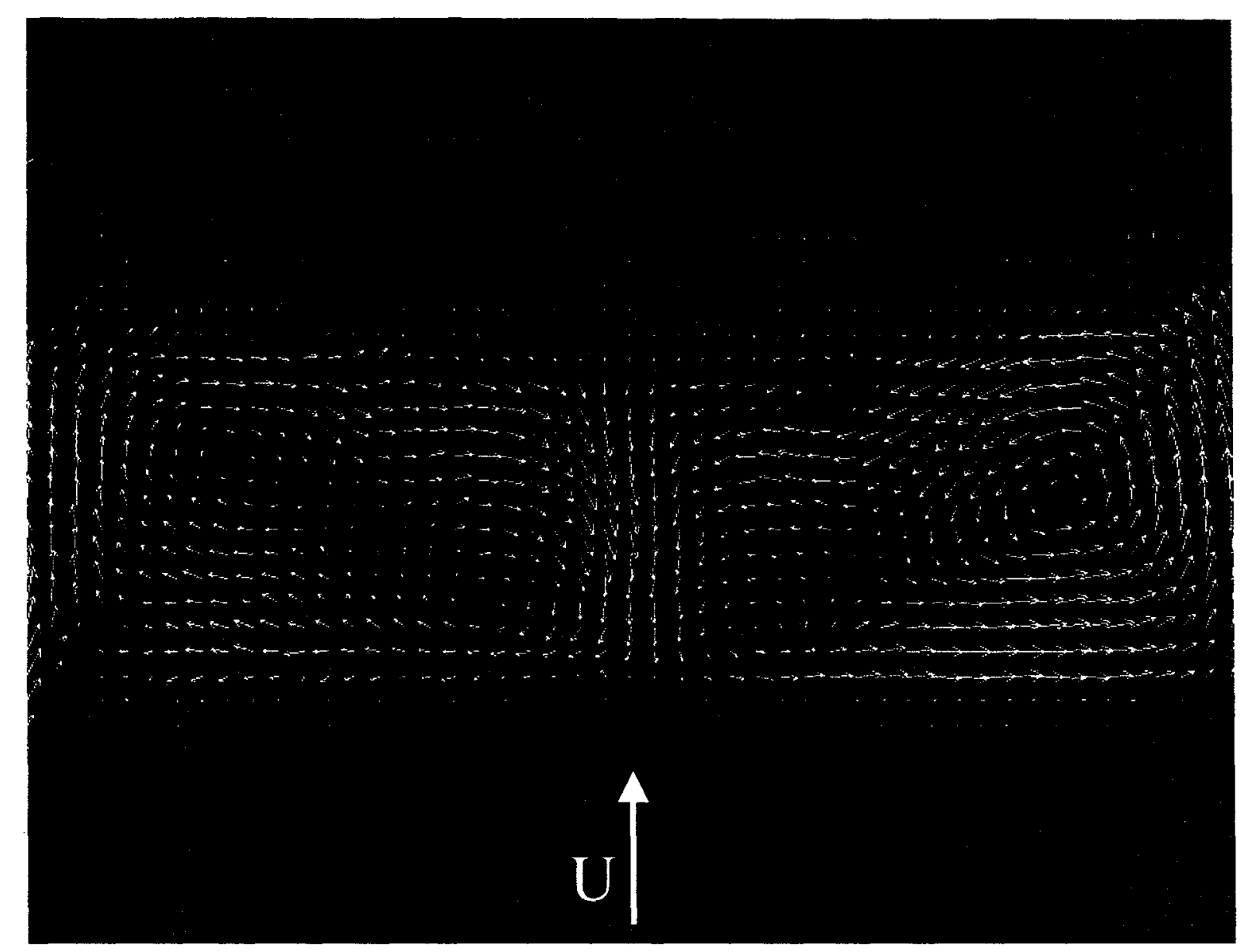

USC March 1999 Contractors meeting 


\section{Velocity field for $D / L=6 \%$ Horizontal plane at $\mathrm{Z} / \mathrm{H}=0.84$}

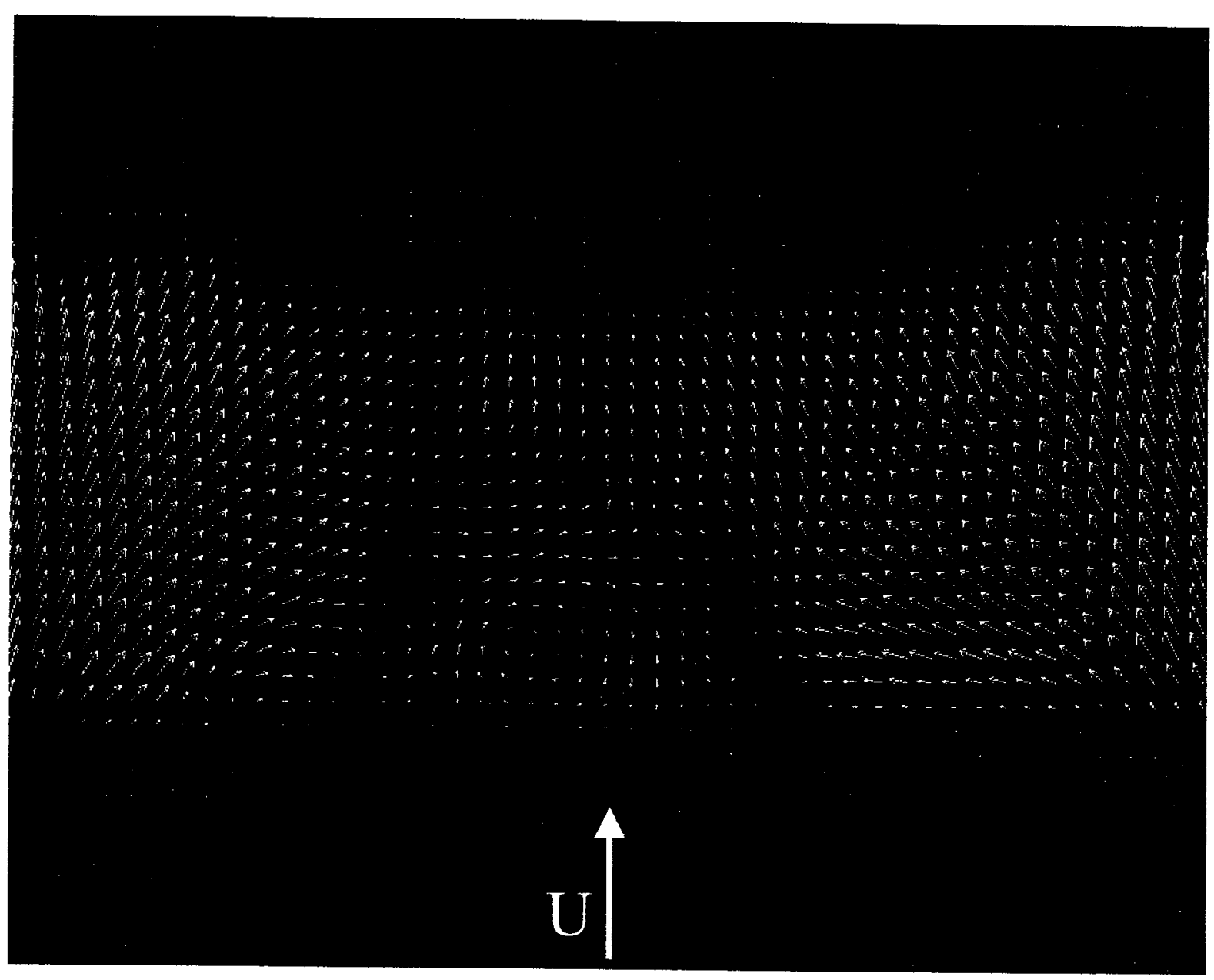


Instantaneous velocity field

Vertical mid-plane, $D \Lambda=23 \%$

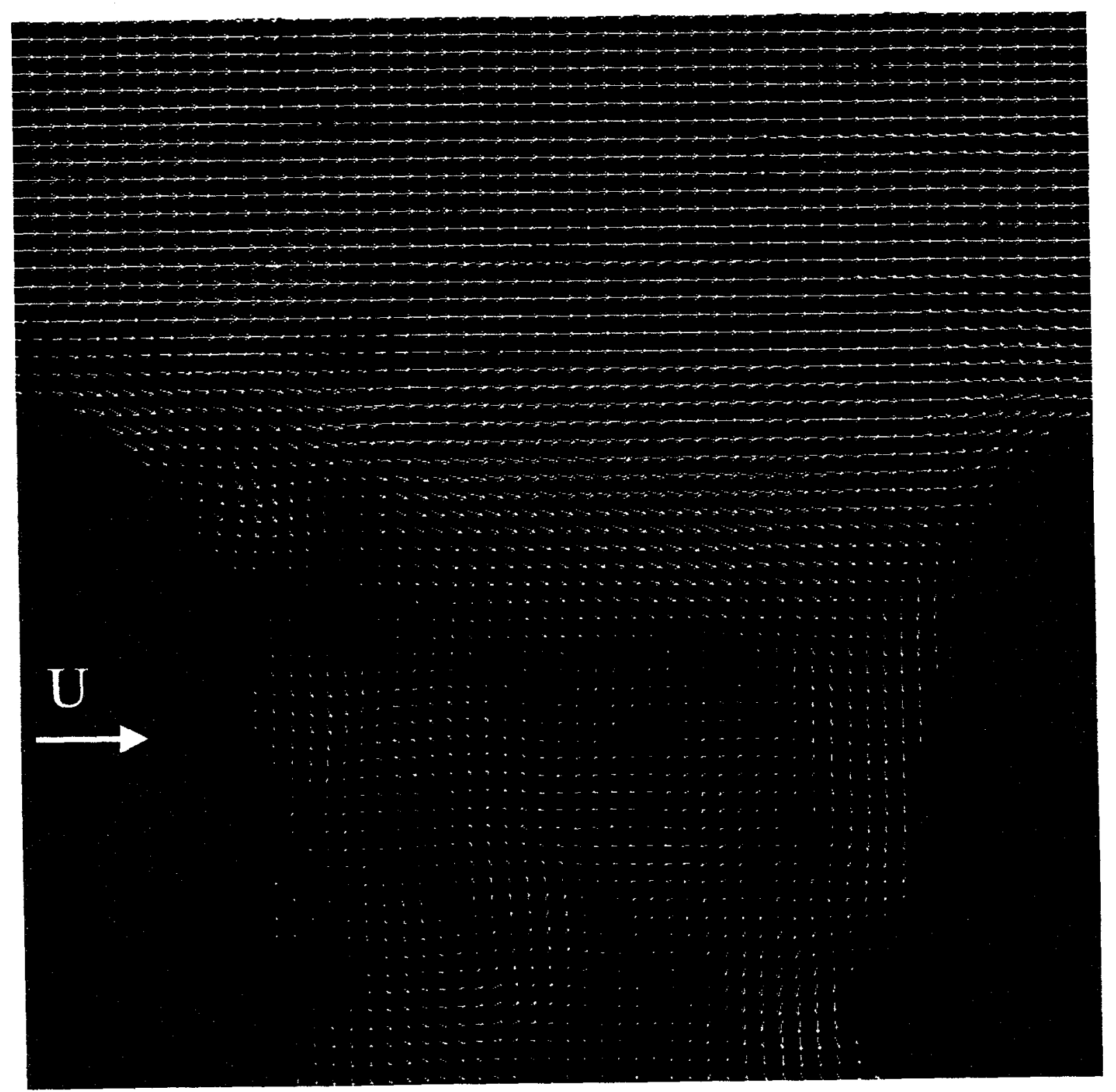

USC March 1999 Contractors meeting 
Instantaneous velocity field
Vertical mid-plane, $D / L=6 \%$

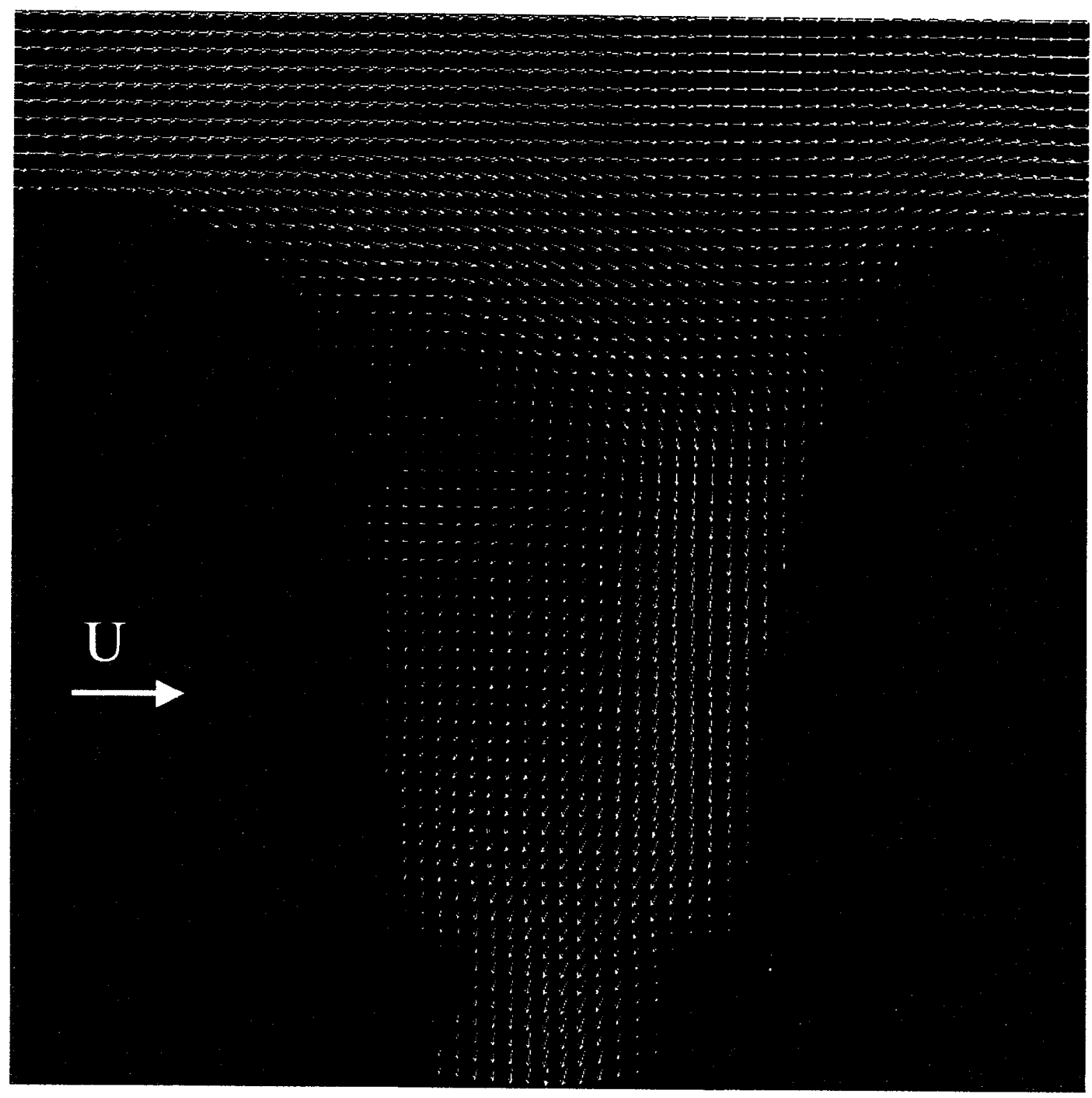

USC March 1999 Contractors meeting 
Aerodynamic Design of Heavy Vehicles

Overview of the Computational Plans (RANS, LES)

\author{
Kambiz Salari \\ Aerosciences and Compressible Fluid Mechanics Dept. 9115 \\ Sandia National Laboratories
}

March 1999

Sandia National Laboratories 


\section{Projected Sandia Milestones, FY99-FY01}

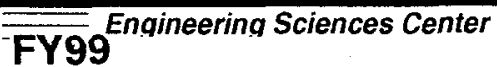

High Reynolds number RANS calculations

Comparison with Texas A\&M 7x10 test

Begin working with NASA/ARC $7 \times 10$ test

Initiate tractor-trailer gap and height mismatch study

Initiate incorporation of LES into SACCARA

\section{FYOO}

Comparison with NASA/ARC $7 \times 10$ test

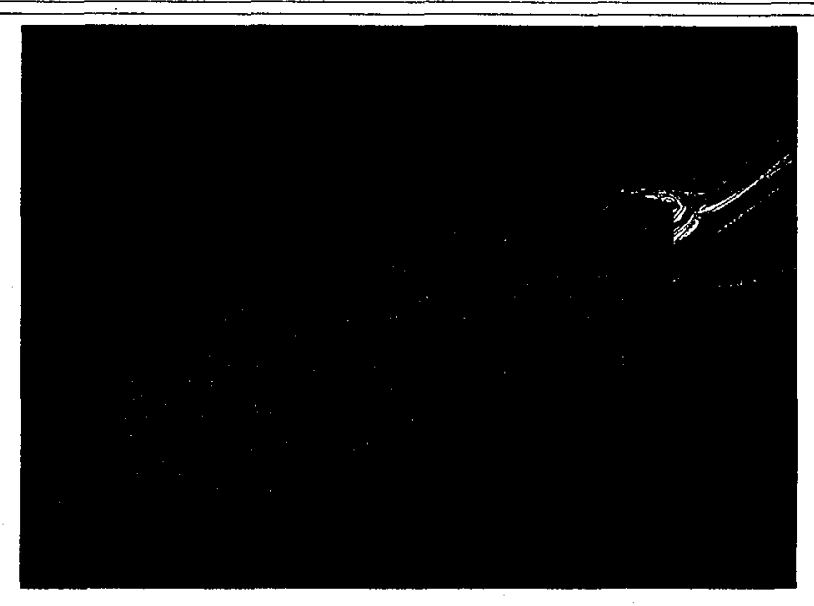

RANS calculation of GTS geometry

Initiate RANS validation process

Continue with incorporation of LES into SACAARA and perform sample calculations

Initiate calculations for the tractor-trailer with gap and height mismatch

FY01

Continue with RANS validation

Continue with LES computation

Continue with gap/height mismatch calculations

Initiate LES validation

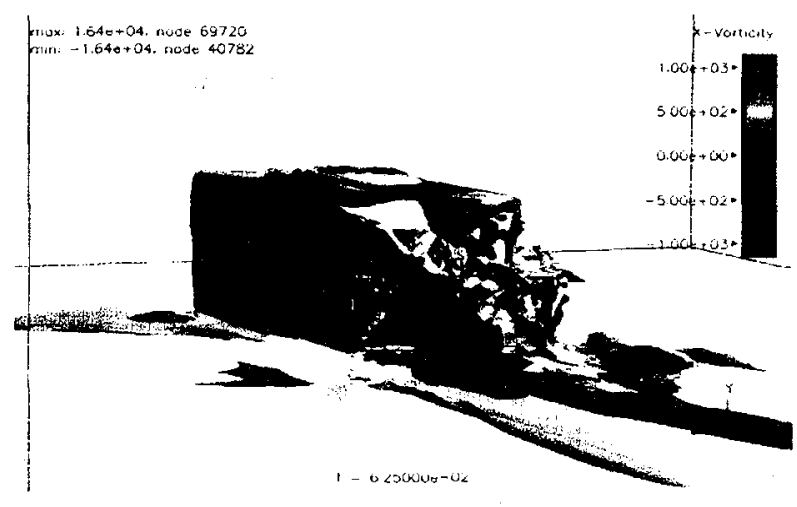

Demo LES calculation of Ahmed geometry 


\section{GTS Flow Simulation}

Ground Transportation System (GTS) vehicle

Texas A\&M 7’x10' low speed tunnel test

Test condition:

Run $=31, \operatorname{Re}=1.6 \times 10^{6}$, Wheels removed

Yaw angle $=0,10$ (deg.)

Free stream velocity $=78(\mathrm{~m} / \mathrm{s})$

Density $=1.17\left(\mathrm{~kg} / \mathrm{m}^{3}\right)$

Static pressure $=99,470.6(\mathrm{~Pa})$

Kinematic viscosity $=1.555 \times 10^{-5}\left(\mathrm{~m}^{2} / \mathrm{s}\right)$ 


\section{Matrix for Grid Convergence Study}

\begin{tabular}{|c|c|c|c|}
\hline Yaw Angle & \multicolumn{3}{|c|}{ Grid Size } \\
\hline & Coarse & Medium & Fine \\
\hline 0 & $\mathrm{X}$ & $\mathrm{X}$ & \\
\hline 10 & $\mathrm{X}$ & $\mathrm{X}$ & \\
\hline
\end{tabular}

Coarse Mesh: 0.5 million nodes, 107 processors

Medium Mesh: 4 million nodes, 246 processors

Fine Mesh: $\quad 32$ million nodes, 1414 processors

All calculations were performed on TeraFlop ASCI Red machine 


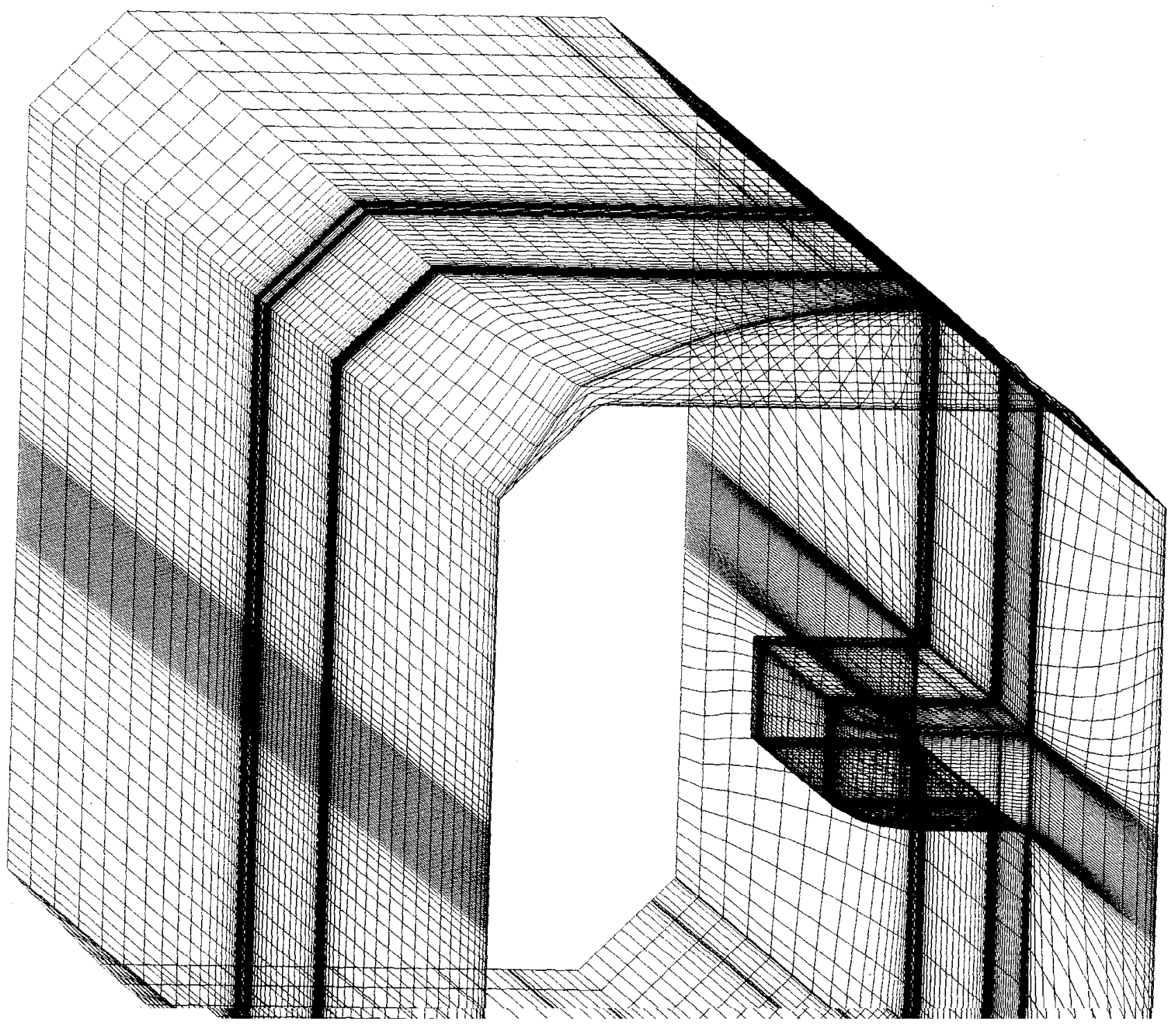




\section{GTS Flow Simulation}
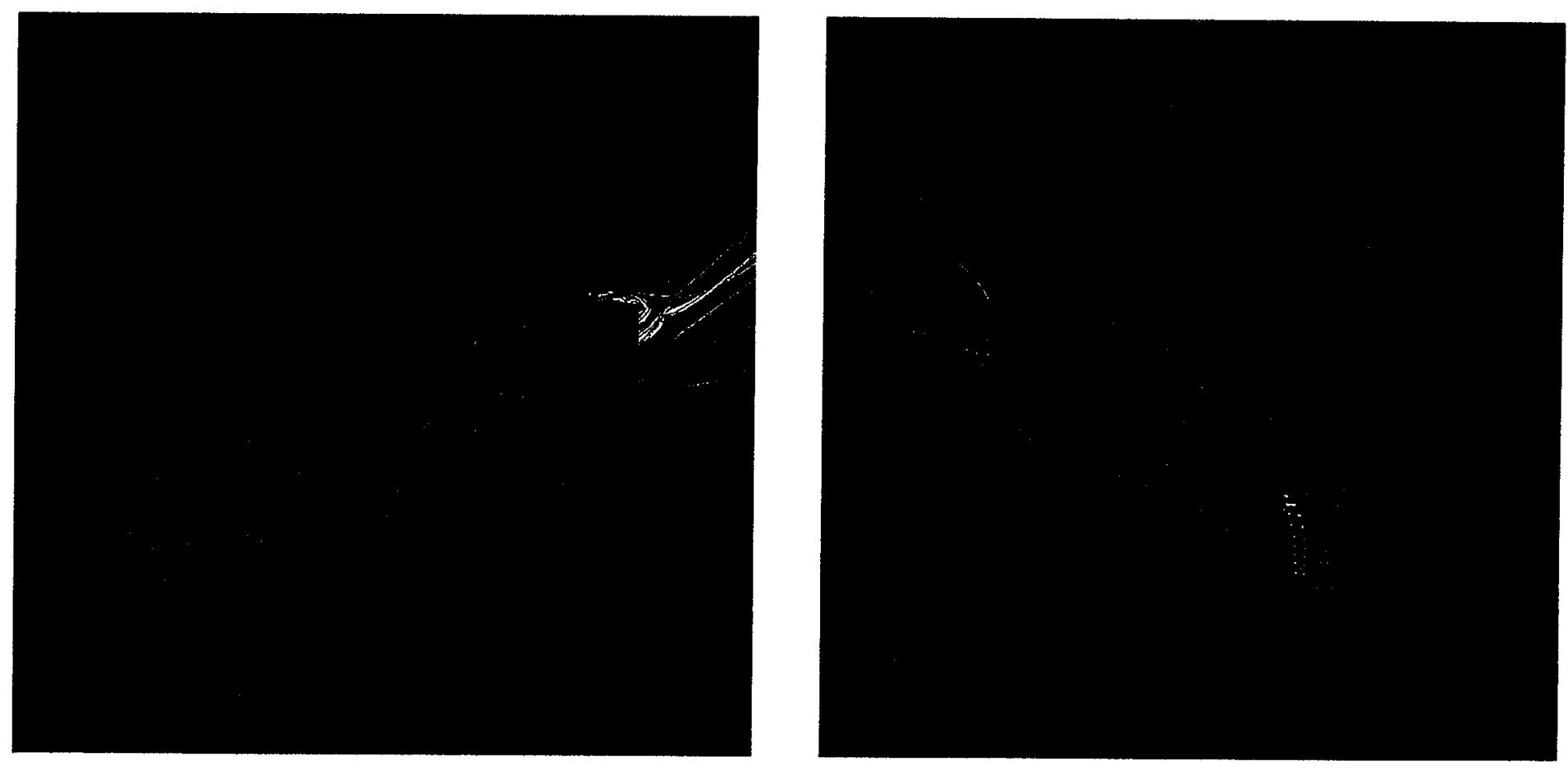


\section{GTS Flow Simulation}

0 degrees yaw angle

$x$-plane cut

Mach contours

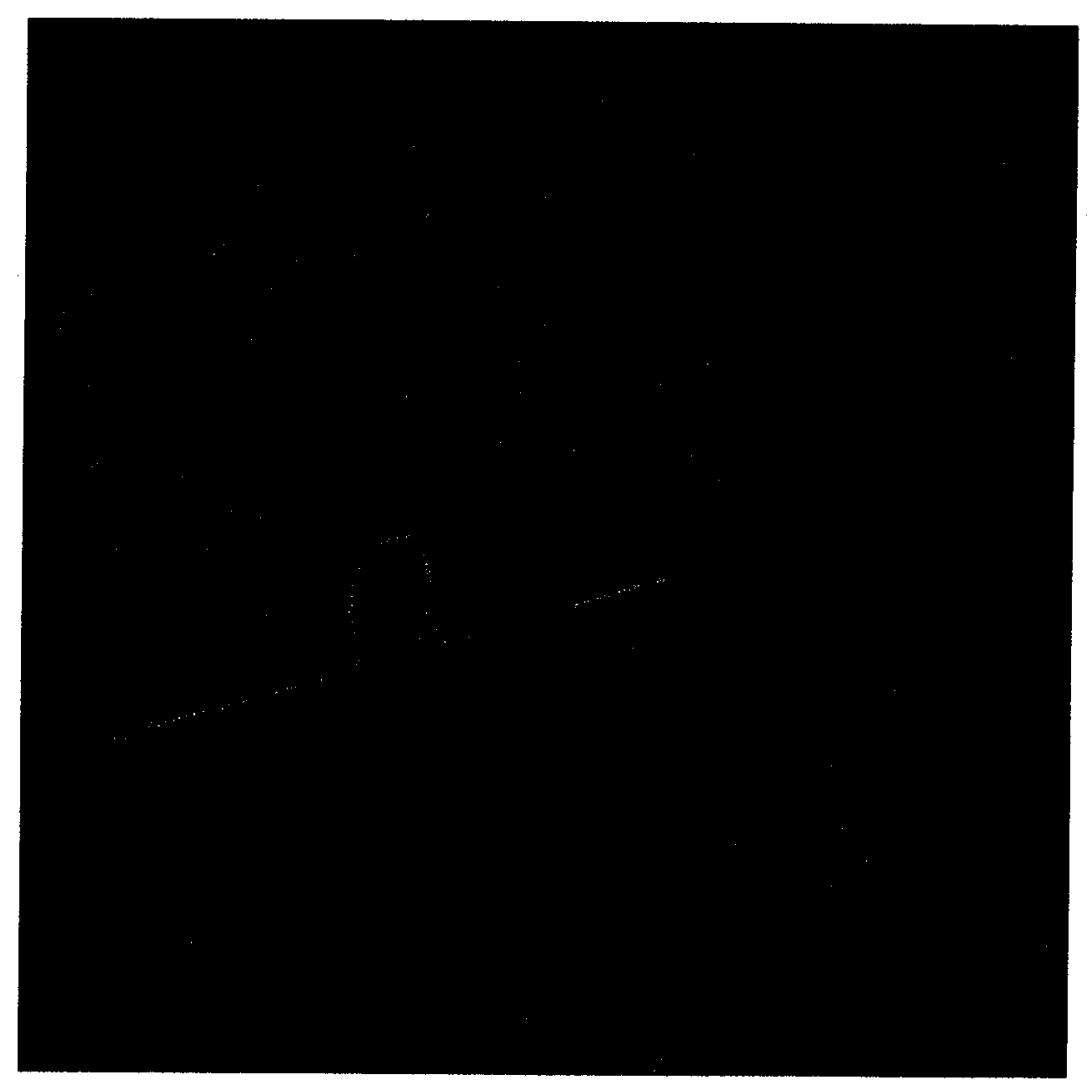




\section{GTS Flow Simulation}

0 degrees yaw angle $x$-plane cut

Mach contours $x=2.5$ meters

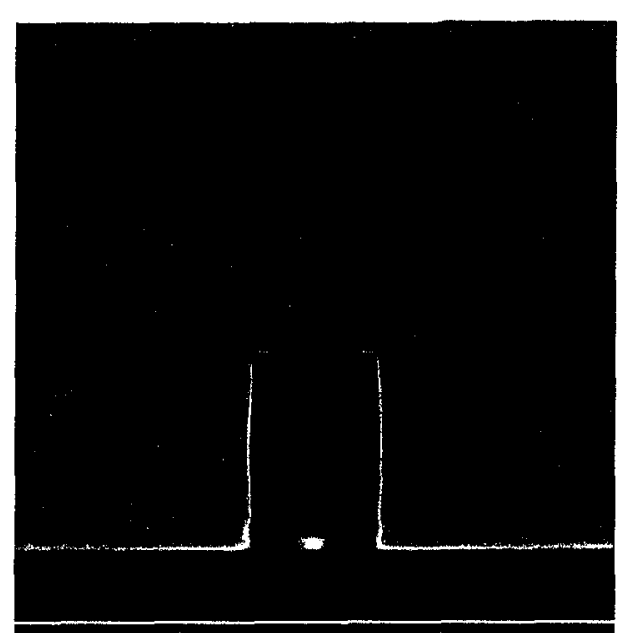

Coarse

Medium
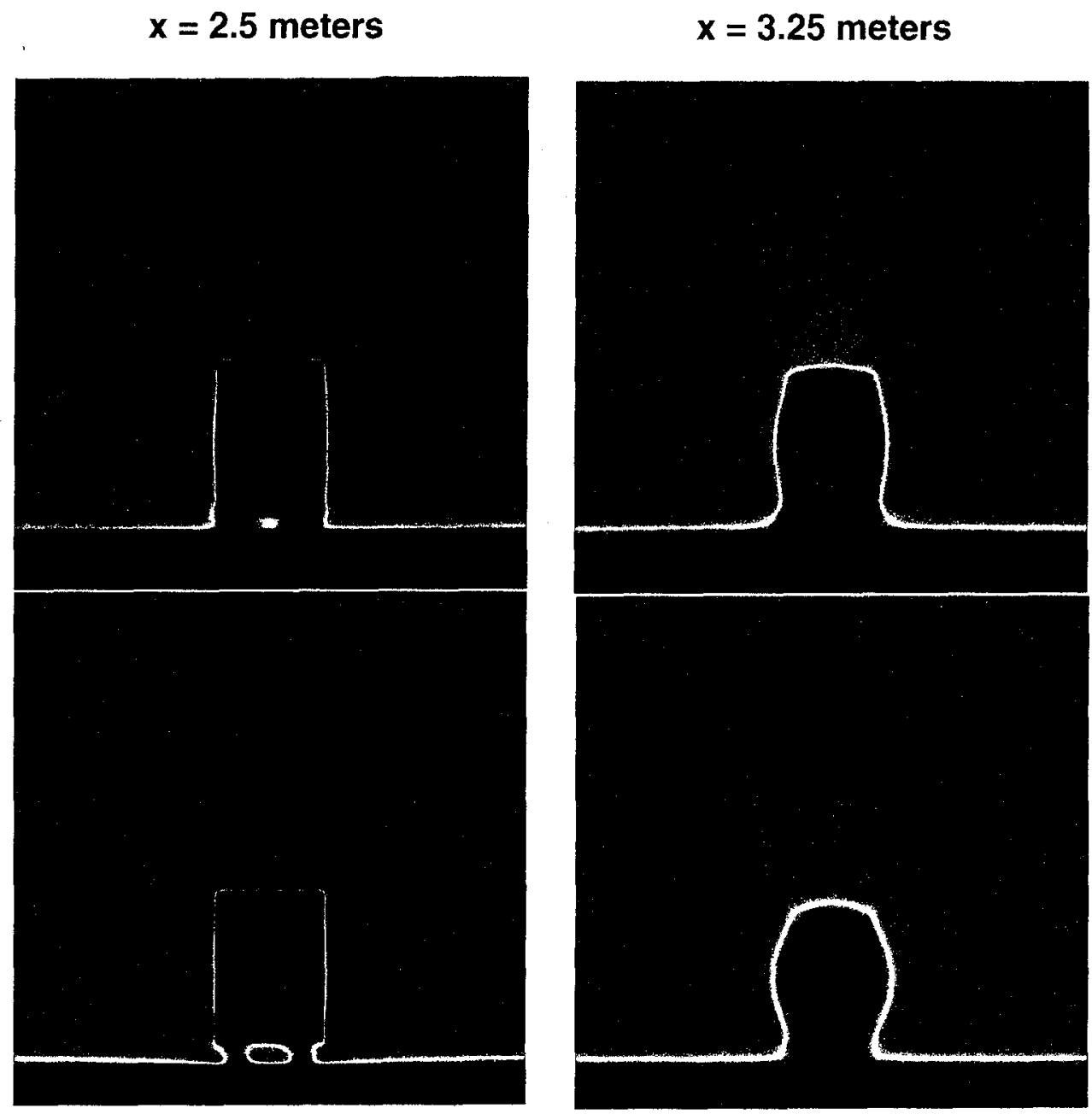


\section{GTS Flow Simulation}

0 degrees yaw angle

$y$-plane cut

Mach contours

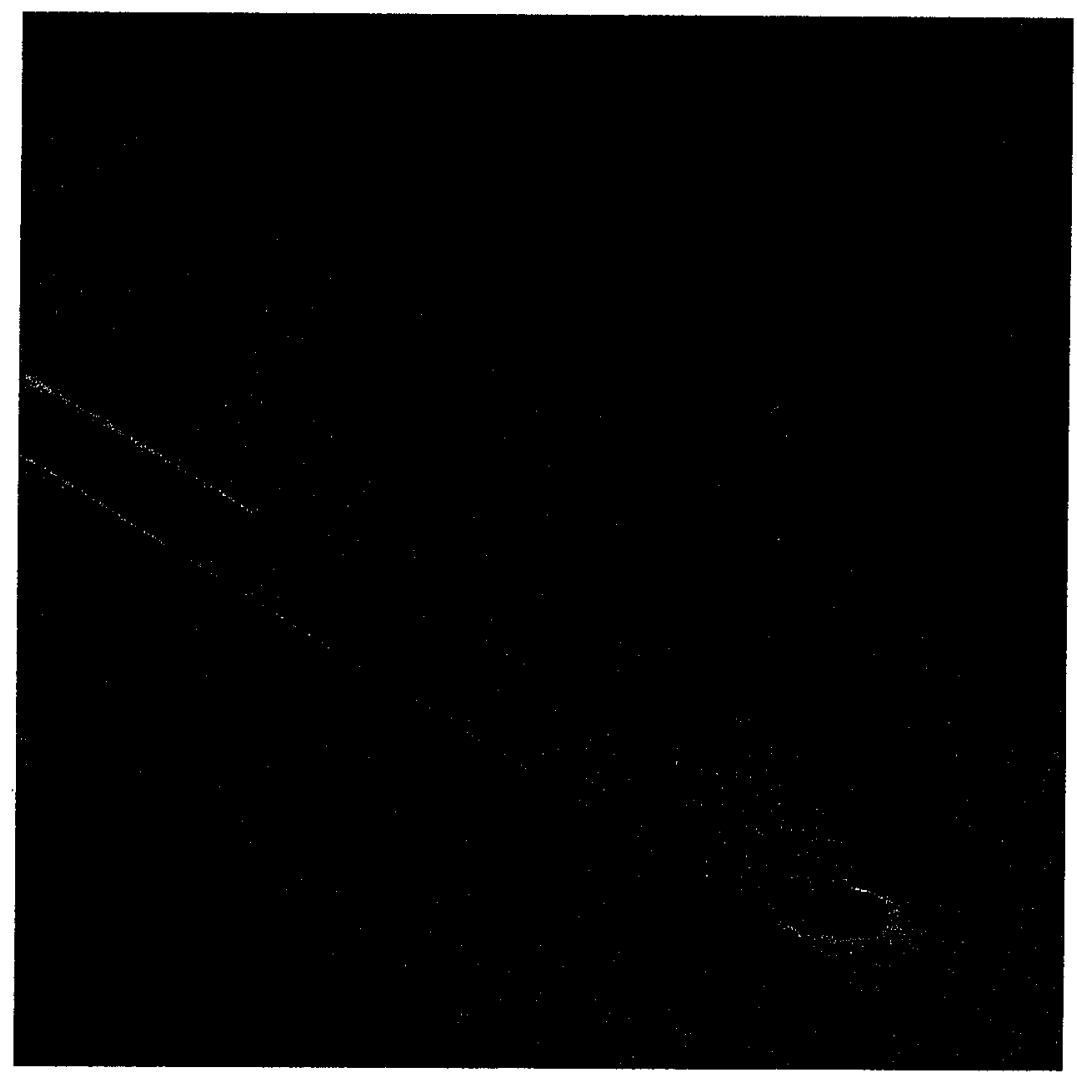




\section{GTS Flow Simulation}

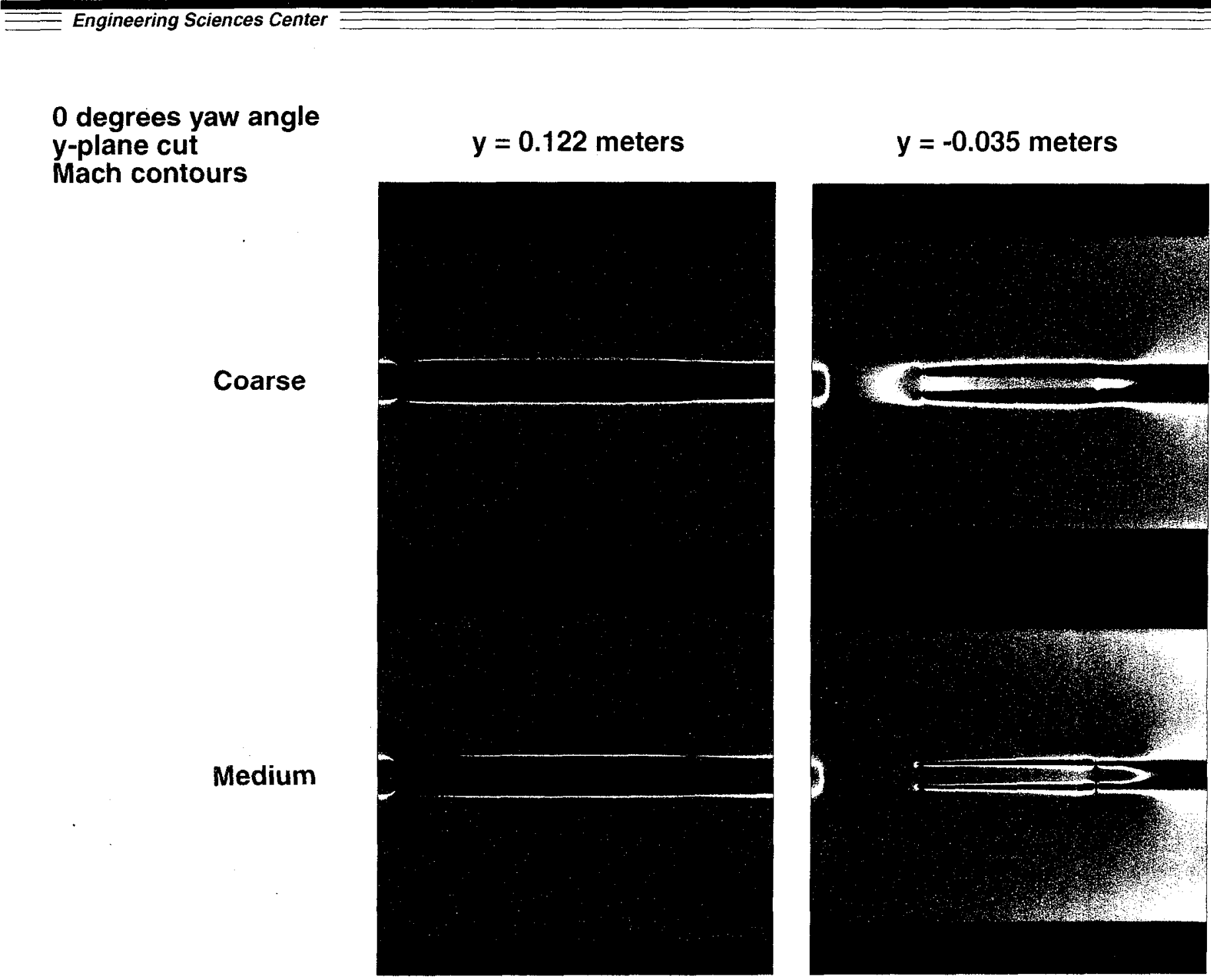




\section{GTS Flow Simulation}

Engineering Sciences Center

0 degrees yaw angle

z-plane cut

Mach contours

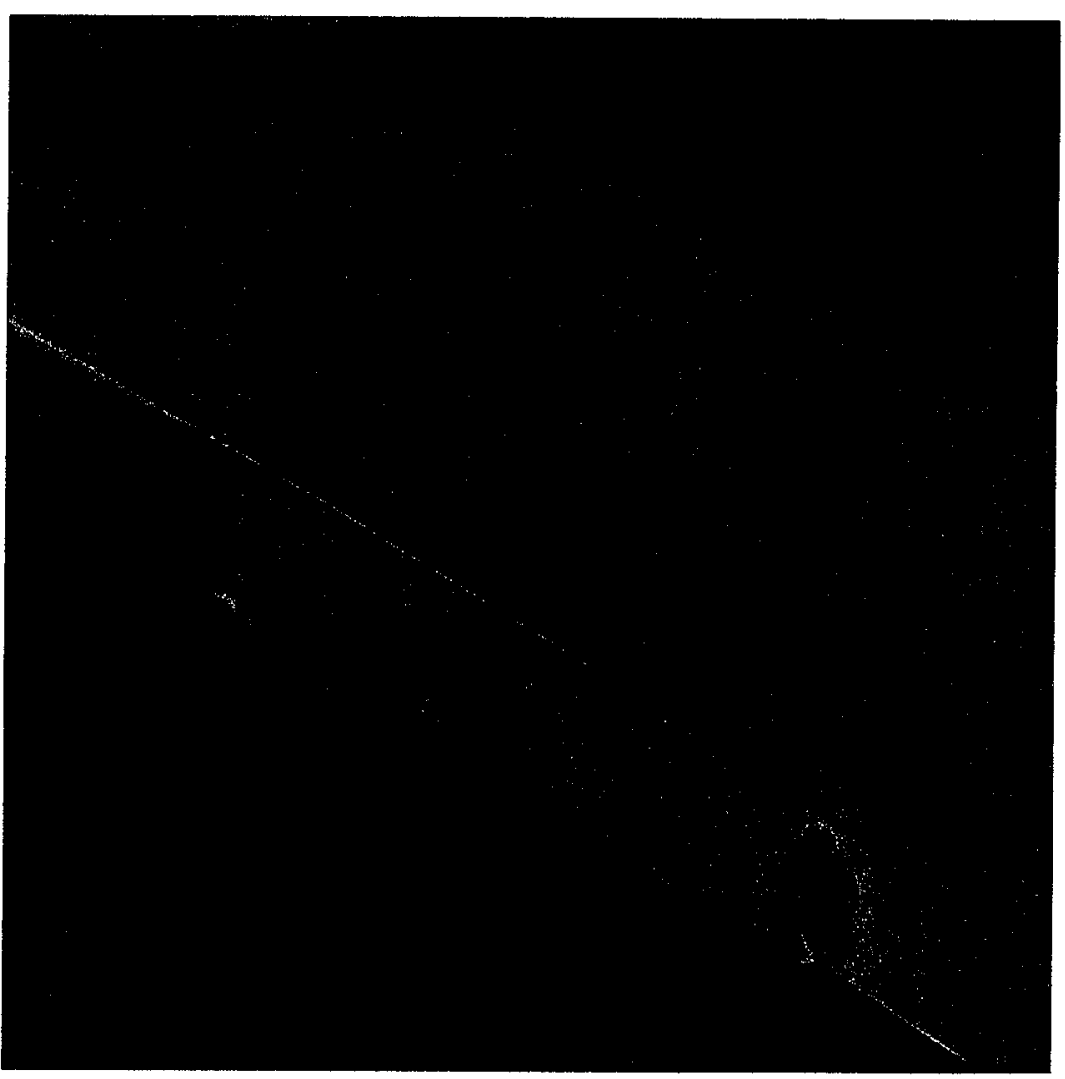




\section{GTS Flow Simulation}

\section{用 snin

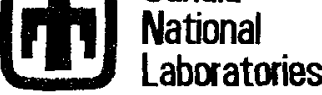

Engineering Sciences Center

0 degrees yaw angle z-plane cut Mach contours

\section{Coarse}

Medium

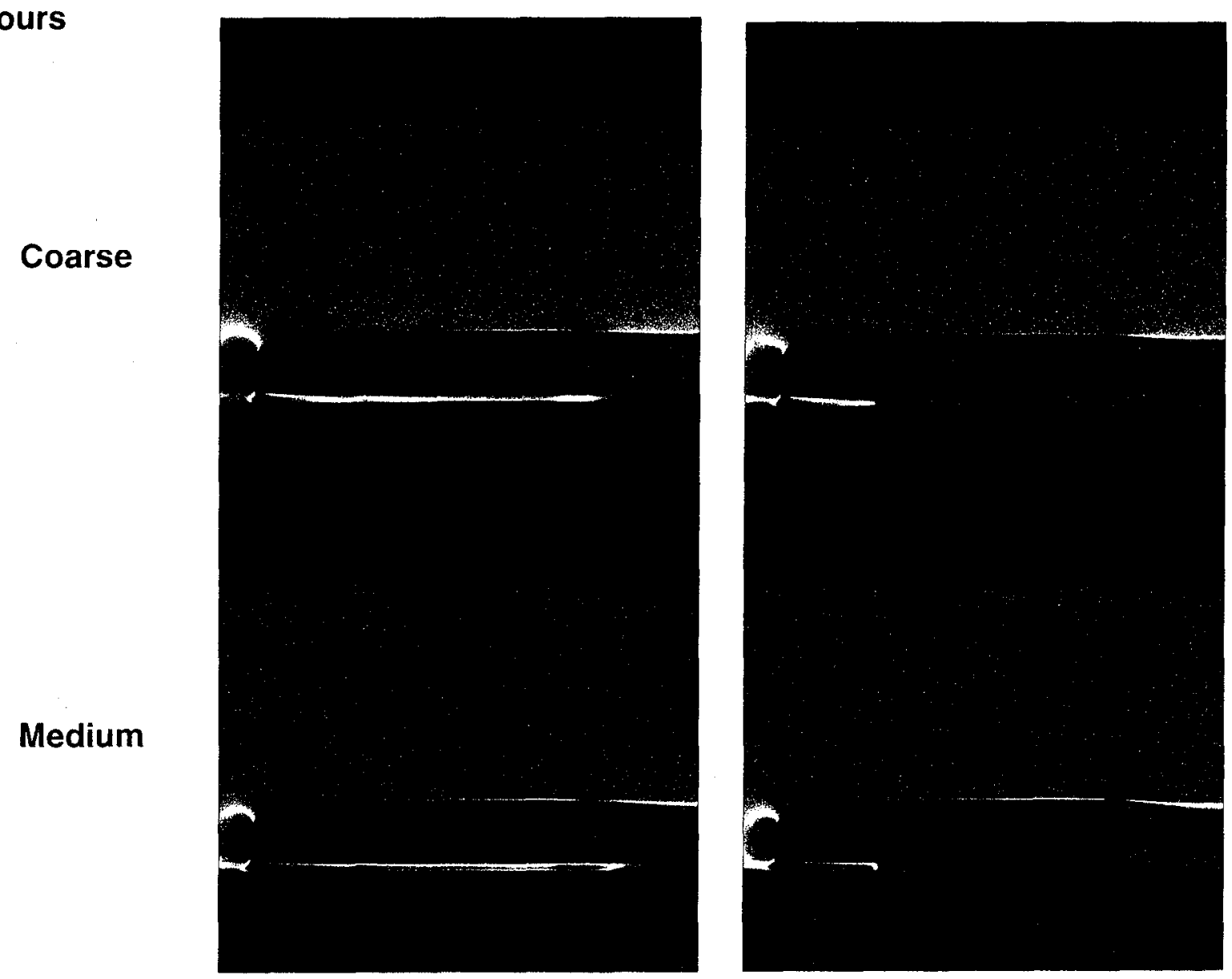

$z=0.1$ meters 


\section{Pressure Distribution on the Surface Coarse to Medium Grid Comparison}

Engineering Sciences Center

0 degrees yaw angle

Coarse
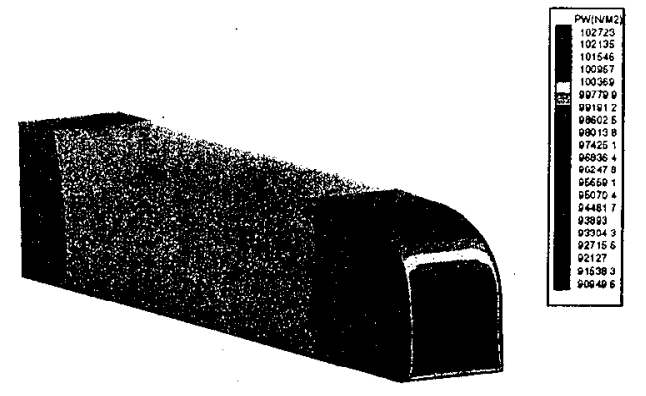

Medium

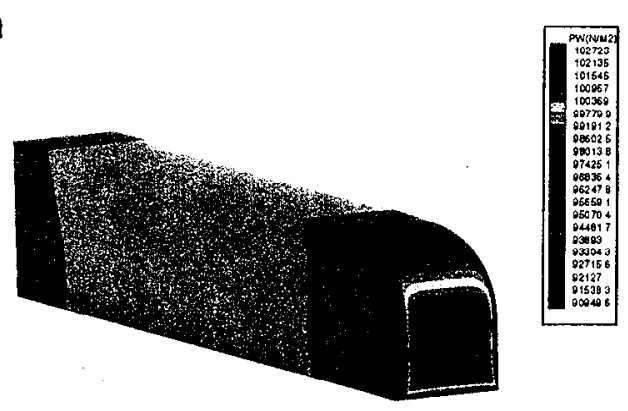

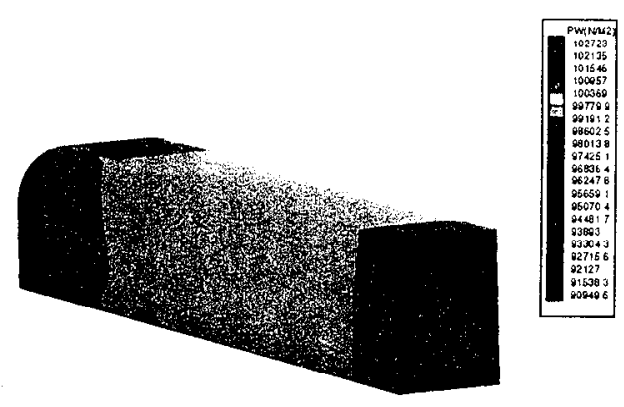

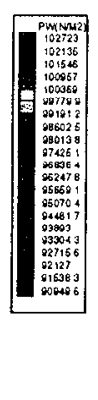




\section{Shear Stress Distribution on the Surface Coarse to Medium grid comparison

Engineering Sciences Center

0 degrees yaw angle

Coarse
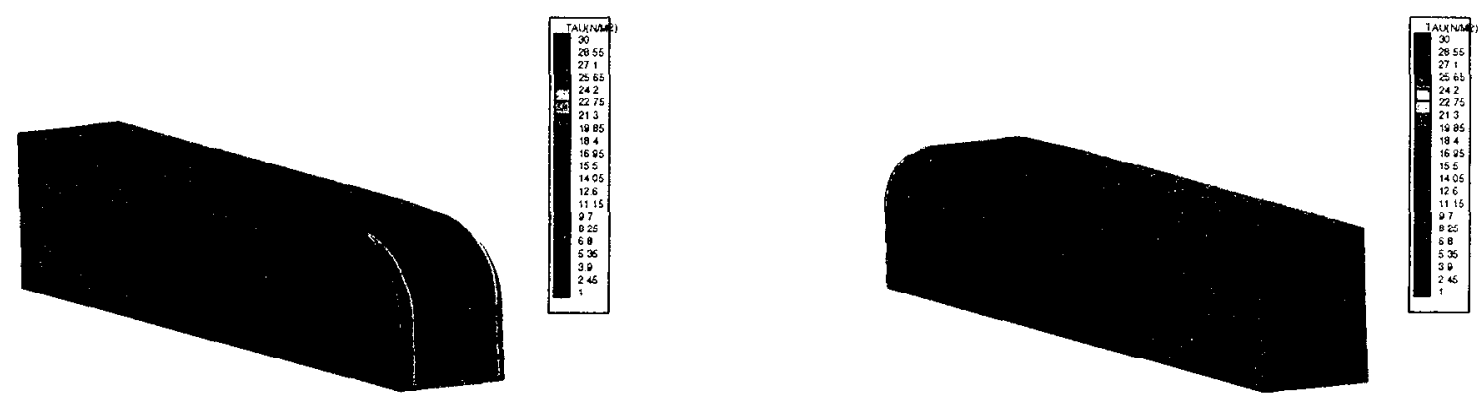

Medium
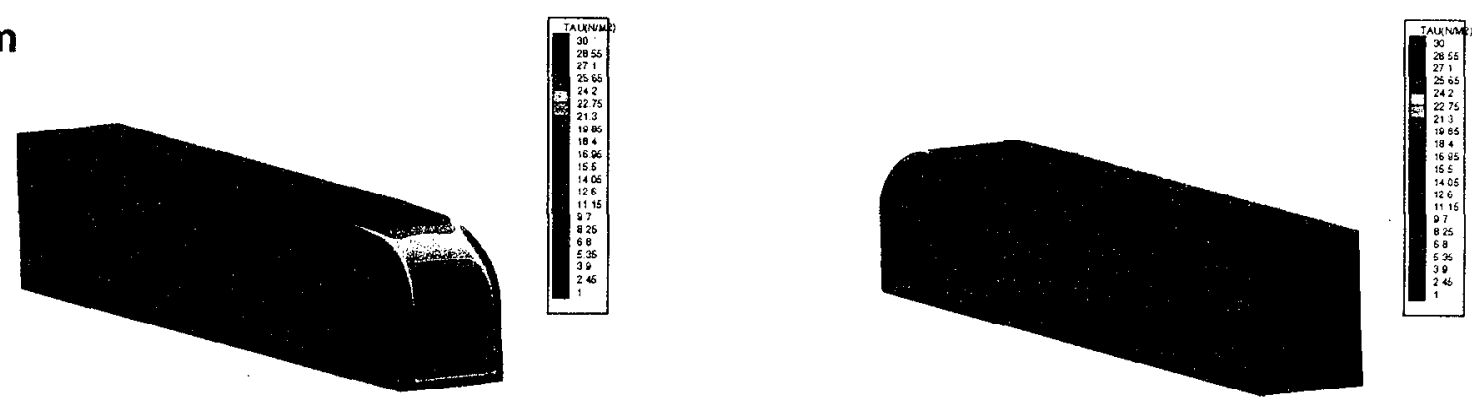


\section{GTS Flow Simulation}

Sinie

田

Laboratories

Engineering Sciences Center

10 degrees yaw angle

$x$-plane cut

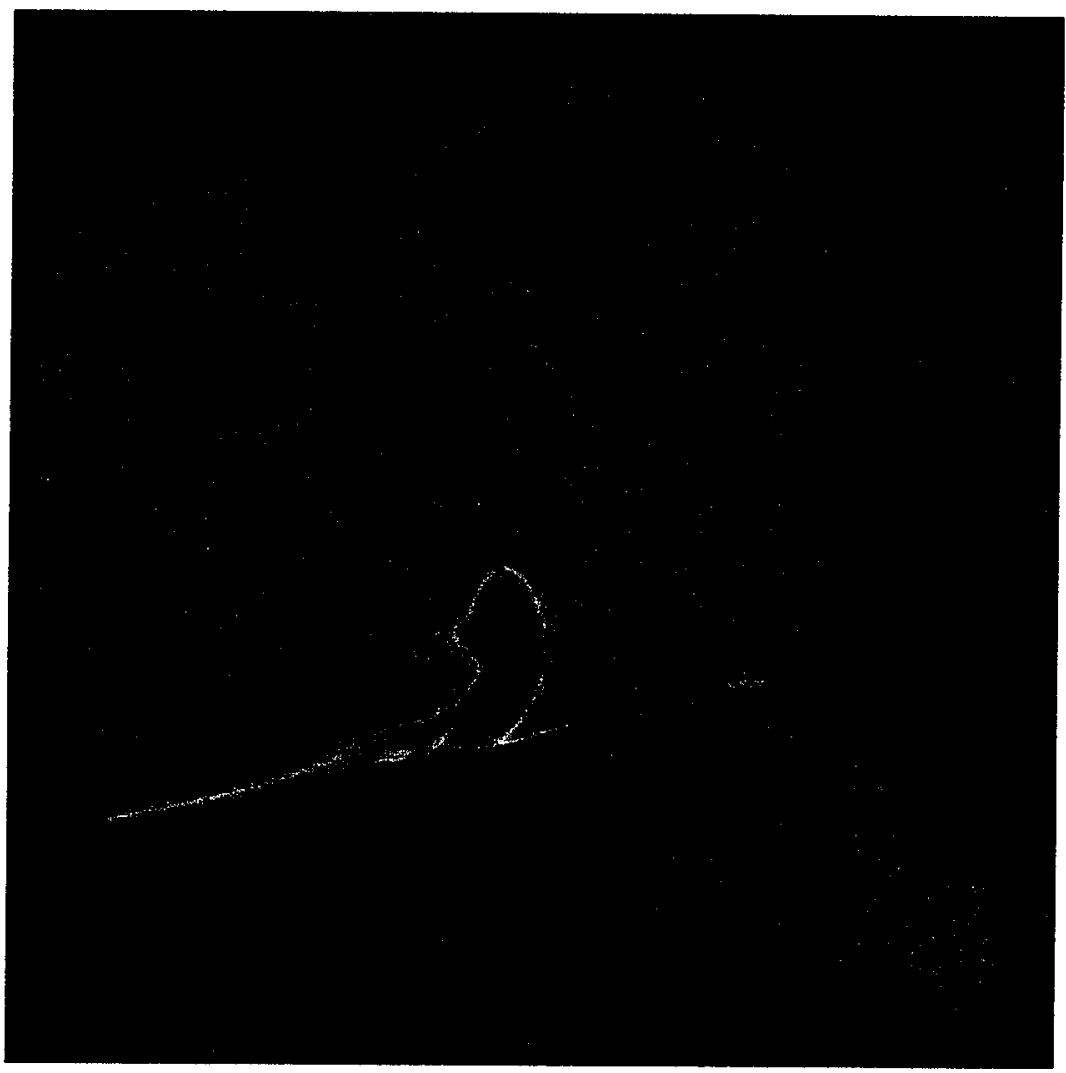




\section{GTS Flow Simulation}

10 degrees yaw angle $x$-plane cut Mach contours

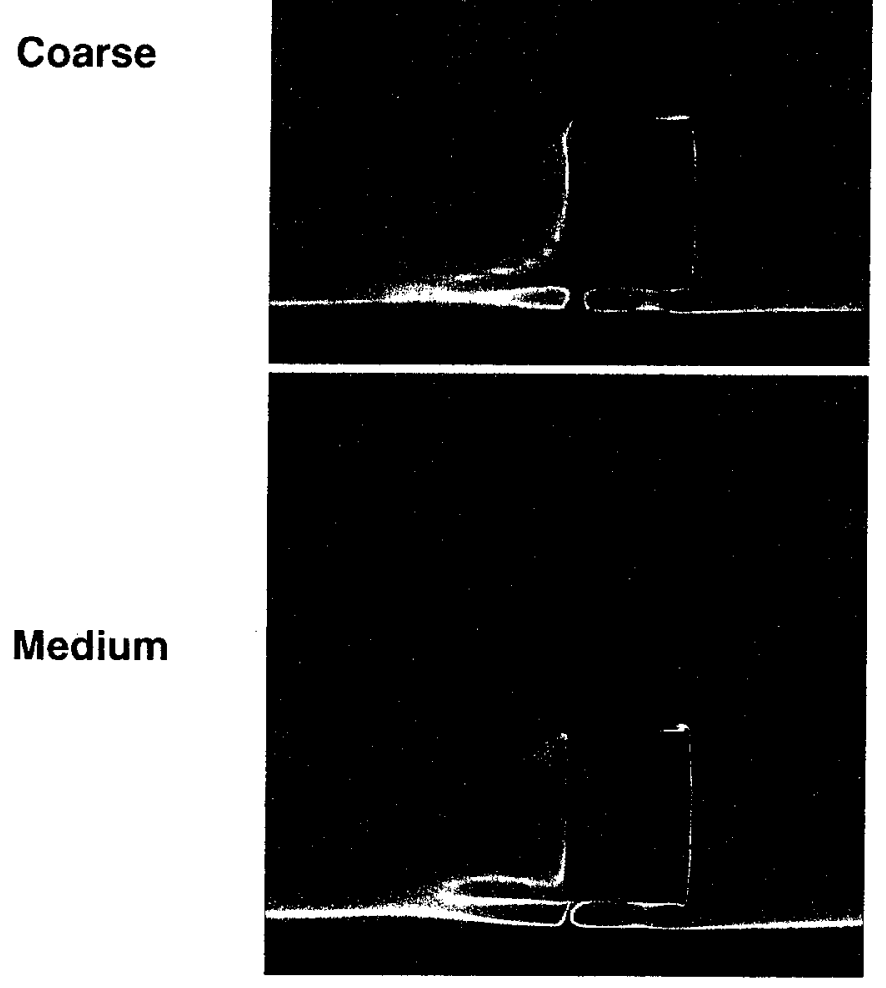

$\mathrm{X}=\mathbf{2 . 5}$ meters

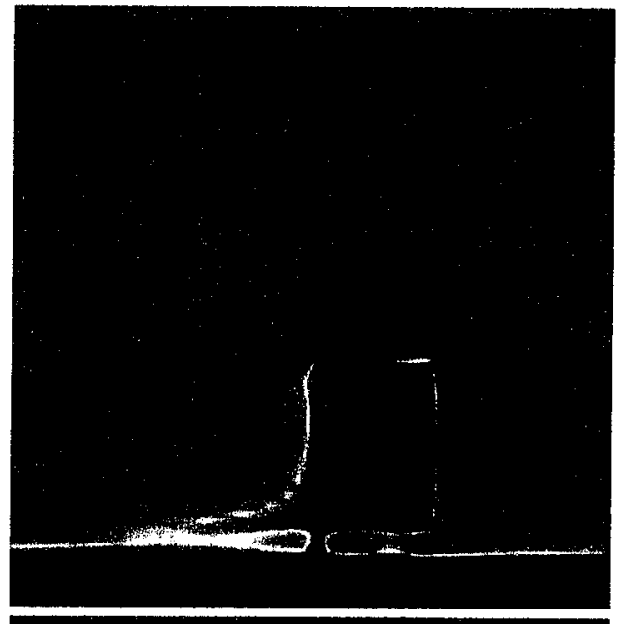

$\mathbf{x}=\mathbf{3 . 2 5}$ meters
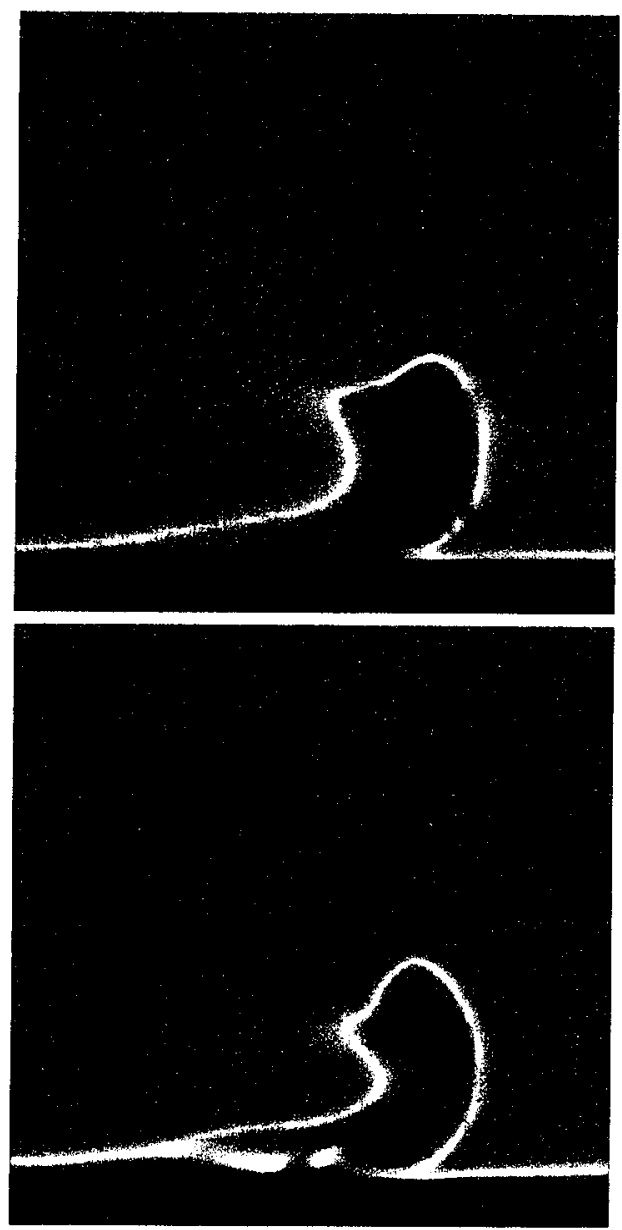


\section{GTS Flow Simulation}

10 degrees yaw angle

$y$-plane cut

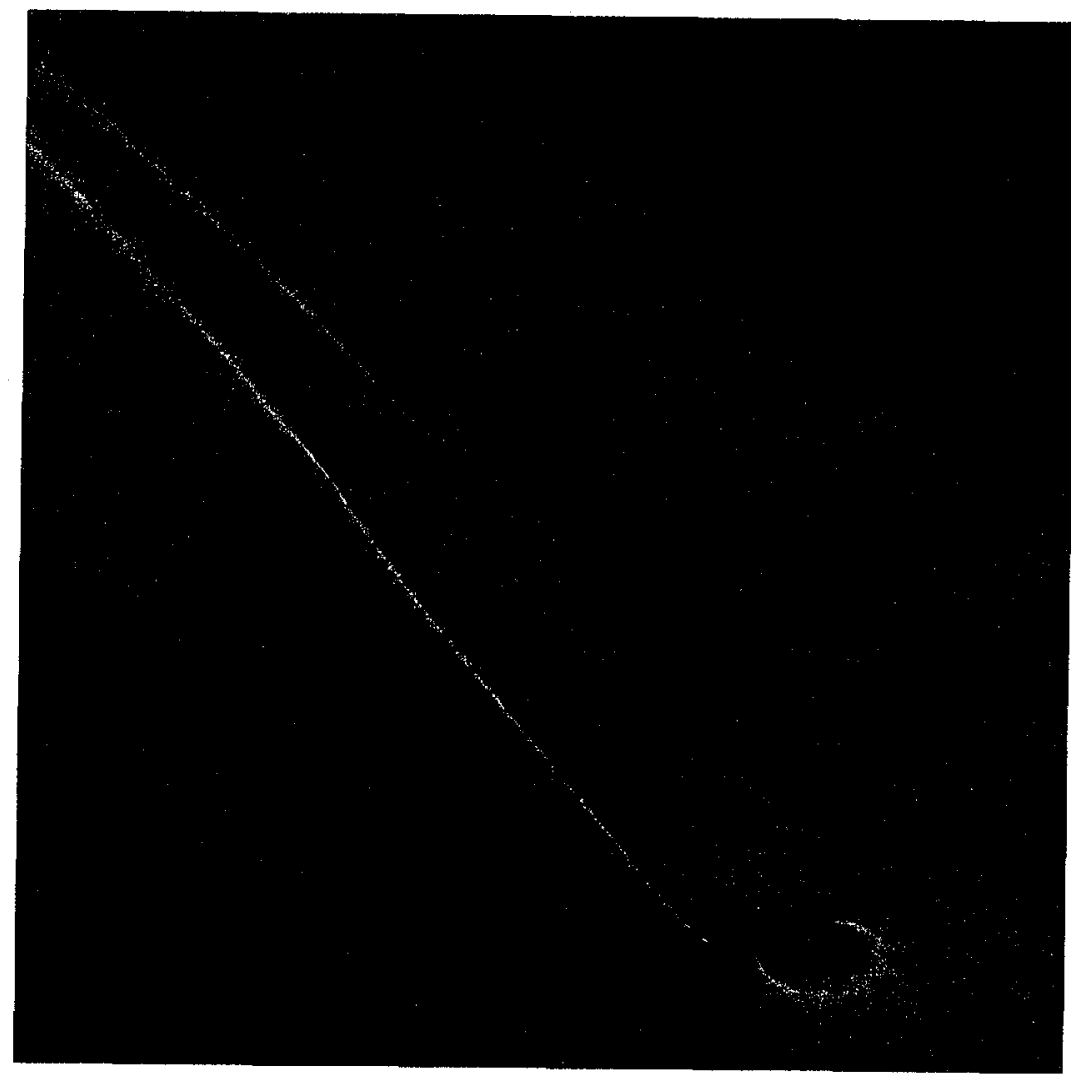




\section{GTS Flow Simulation}

Engineering Sciences Center

10 degrees yaw angle

y-plane cut

Mach contours

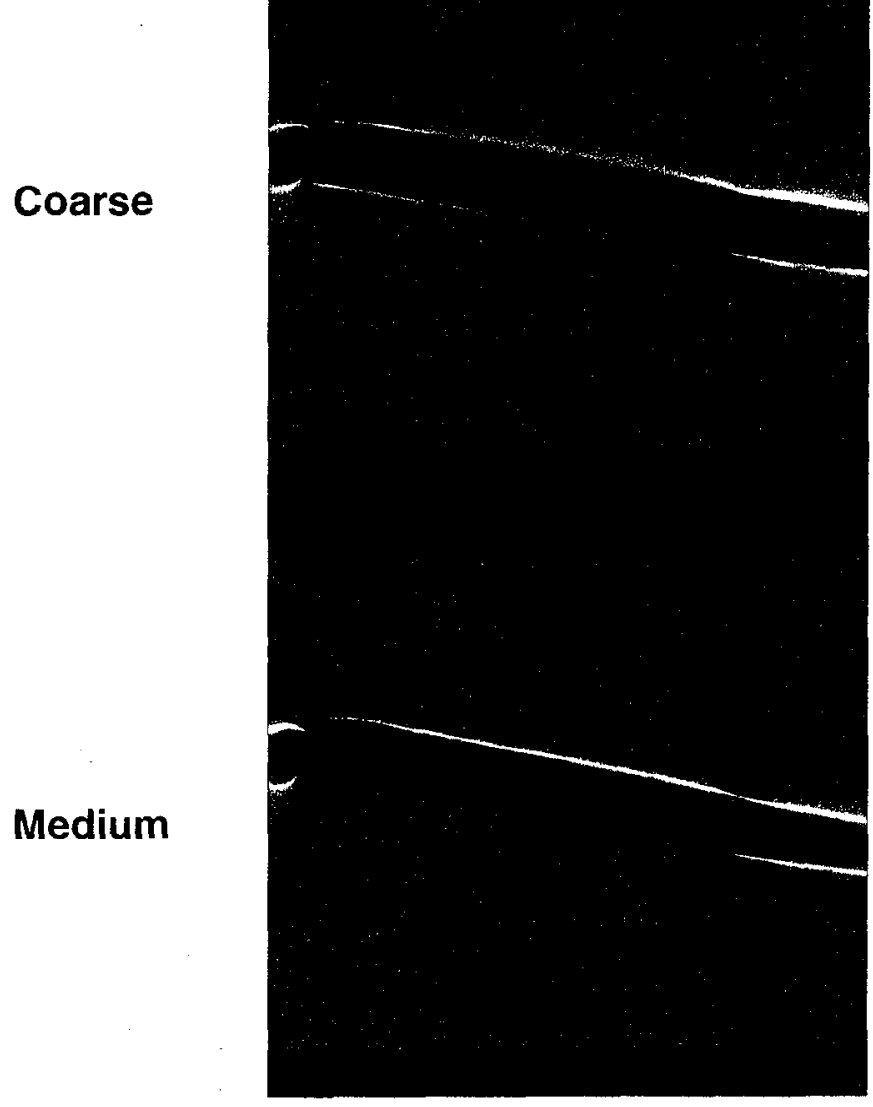

$y=-0.035$ meters

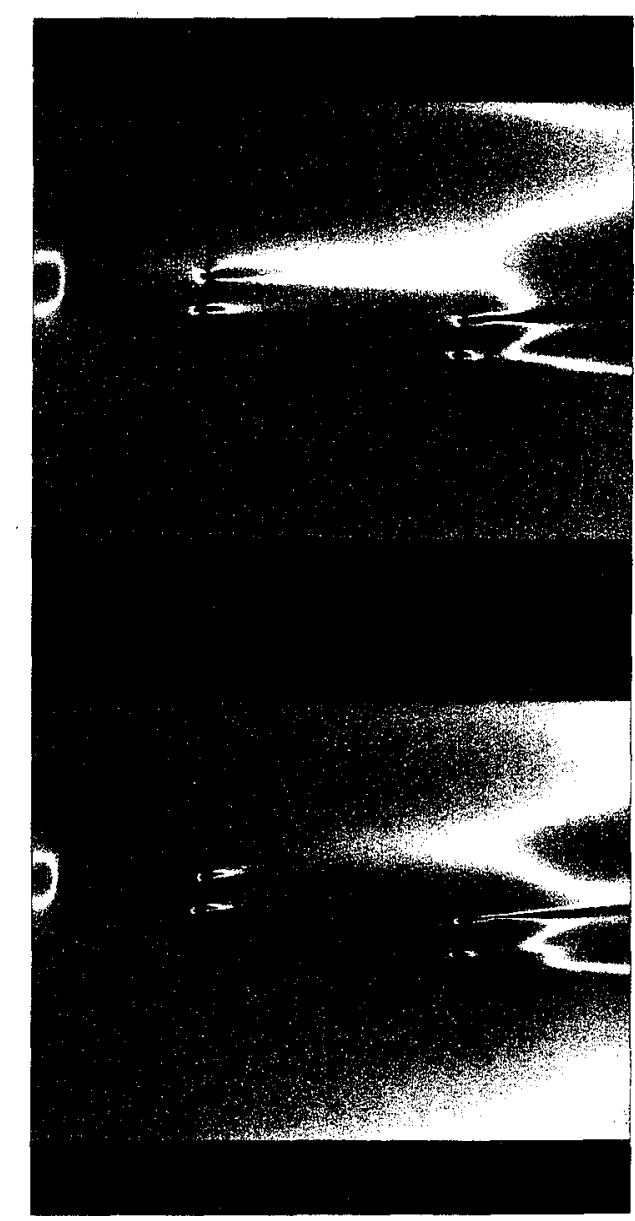




\section{GTS Flow Simulation}

10 degrees yaw angle

z-plane cut

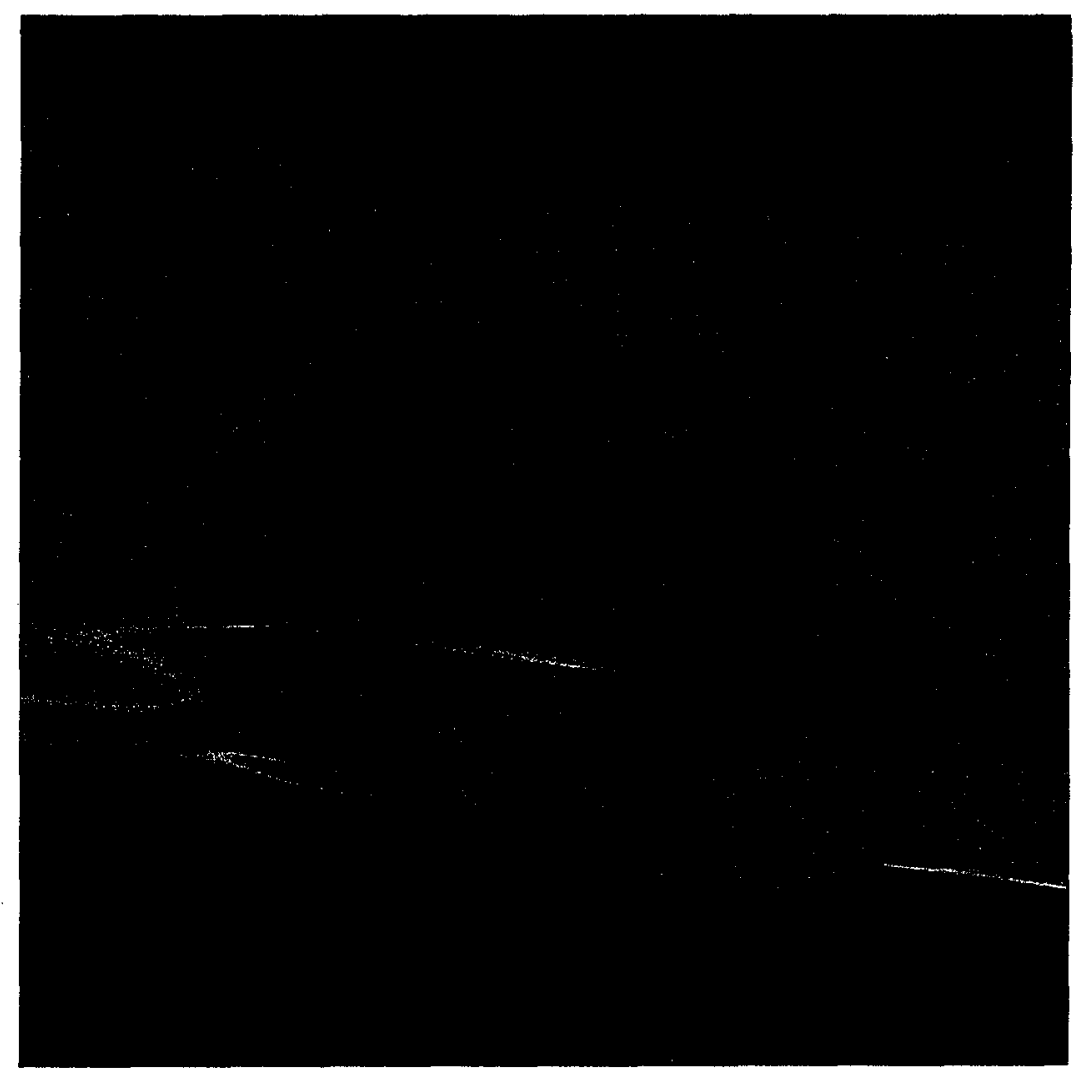




\section{GTS Flow Simulation}

10 degrees yaw angle z-plane cut Mach contours

Coarse

Medium

Coarse

$$
z=0.07 \text { meters }
$$
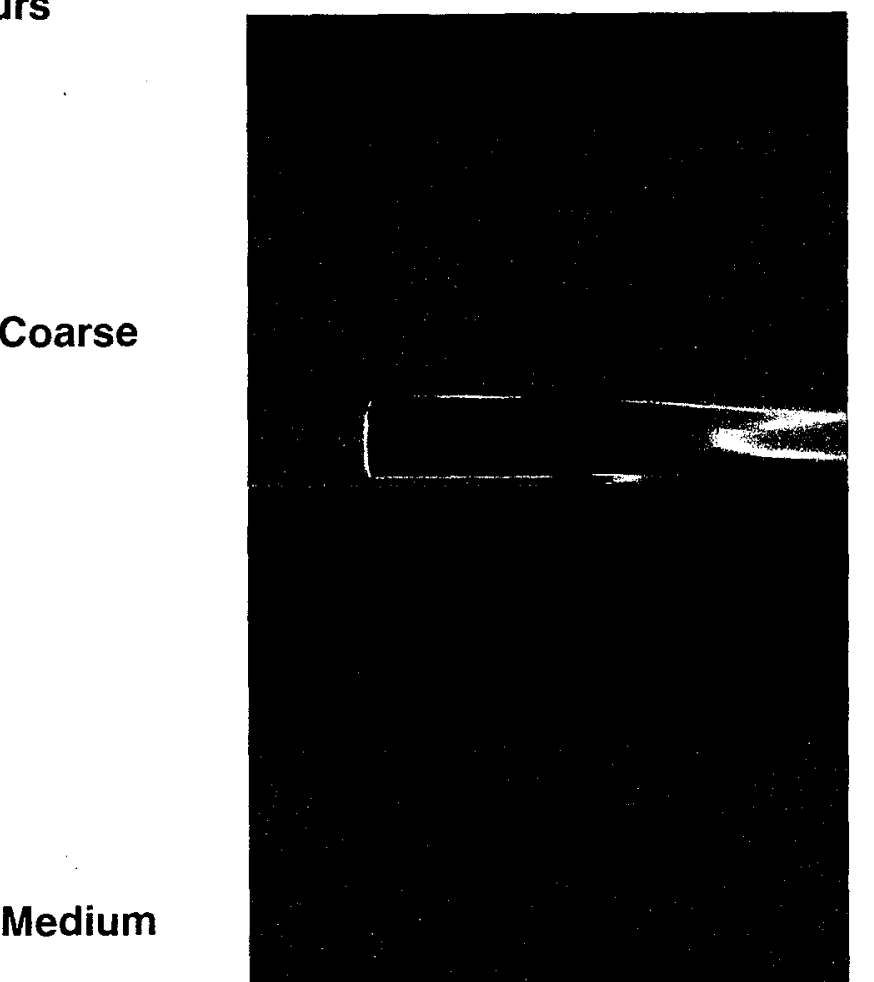

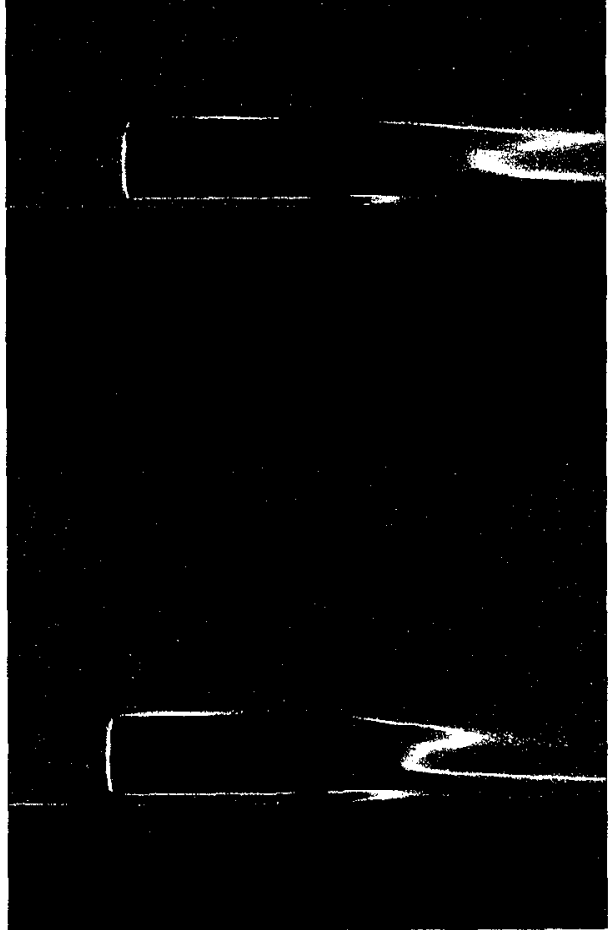

$z=0.215$ meters

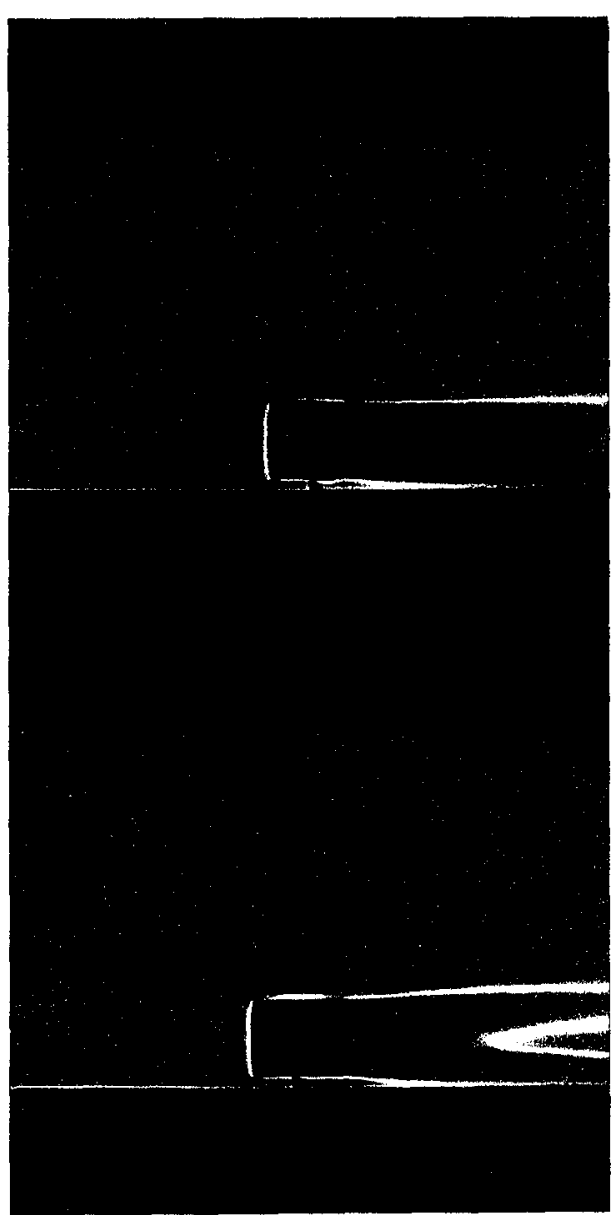




\section{GTS Flow Simulation}

Engineering Sciences Center

10 degrees yaw angle

isoSurface $u=-0.001(\mathrm{~m} / \mathrm{s})$

Coarse

Medium
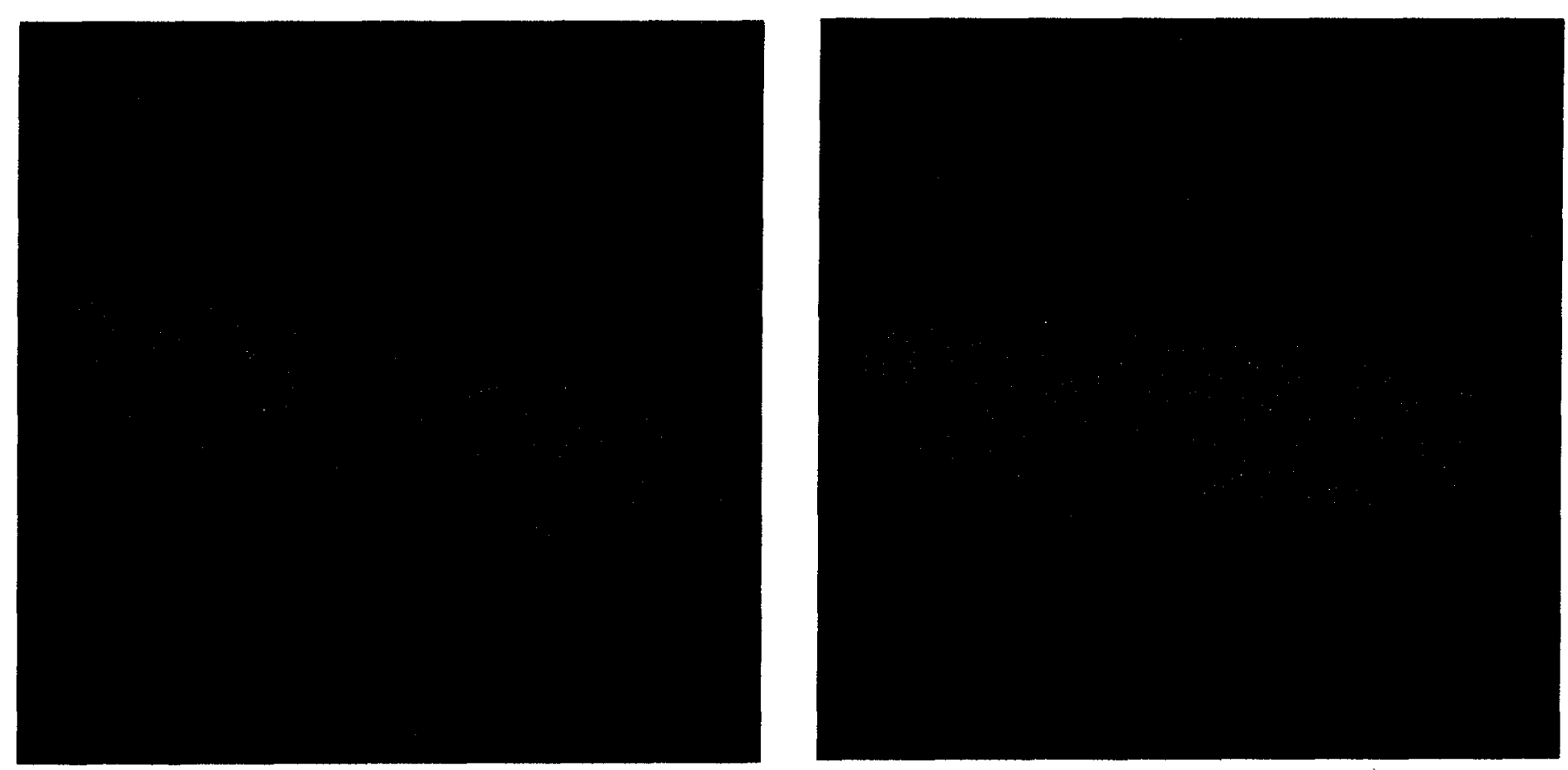


\section{Pressure Distribution on the Surface Coarse to Medium grid comparison}

10 degrees yaw angle
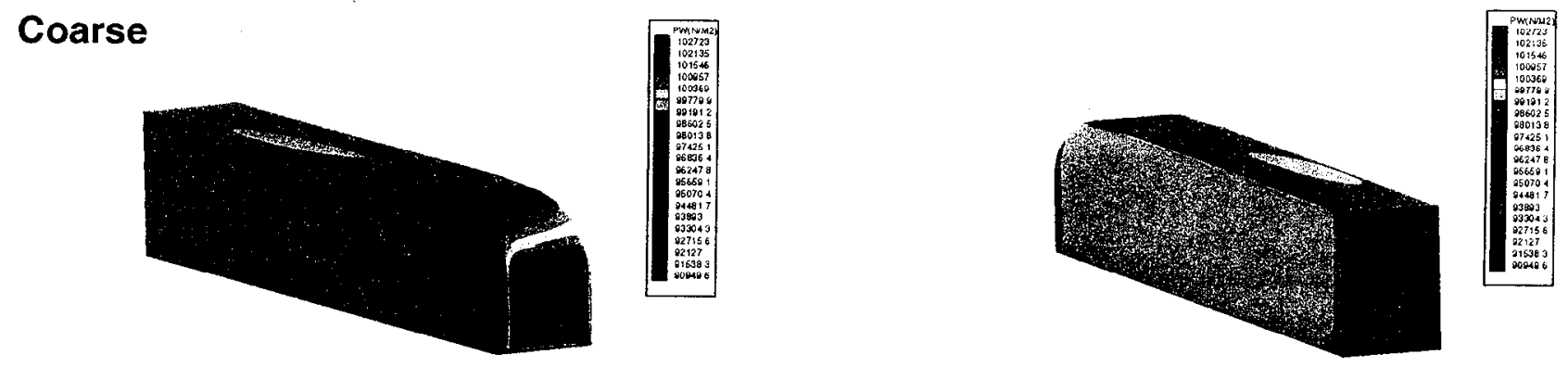

Medium
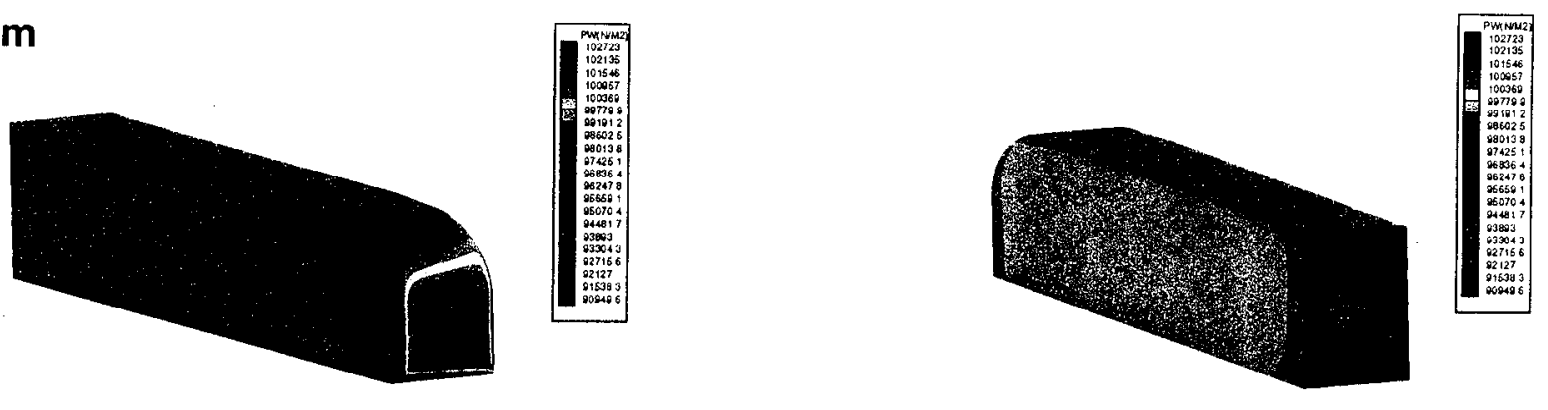


\section{Shear Stress Distribution on the surface Coarse to Medium grid comparison}

10 degrees yaw angle

Coarse
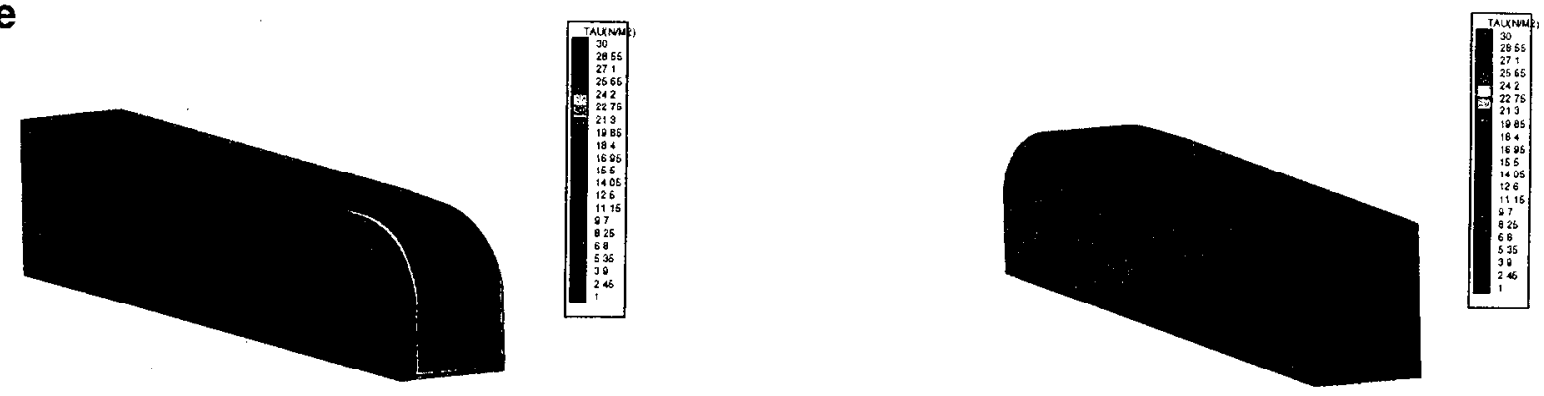

Medium
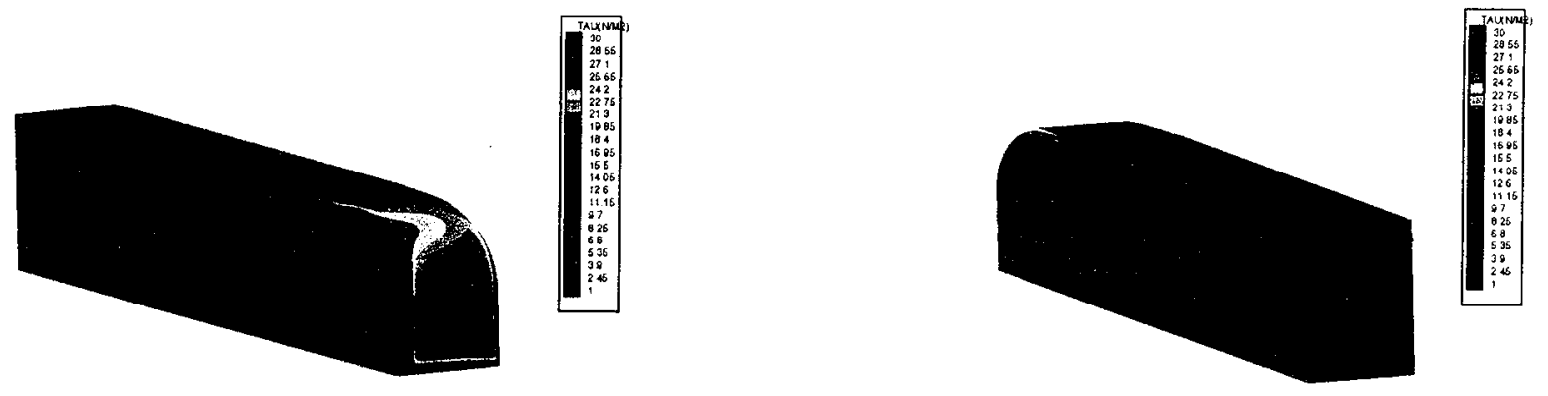


\section{Flow Simulation Around Ahmed-Body GILA LES Computation (M. Christon)}

$\overline{A h m e d-b o d y ~} 30^{\circ}$ slant, Re $=4.29 \times 10^{6}$

Experimental drag coefficient, $\mathrm{C}_{\mathrm{w}}=\mathbf{0 . 3 7 8}$

Predicted short time drag coefficient, $\mathrm{C}_{\mathrm{w}}=\mathbf{0 . 3 8 6}$

Grid size: about 800,000 elements, 128 processors

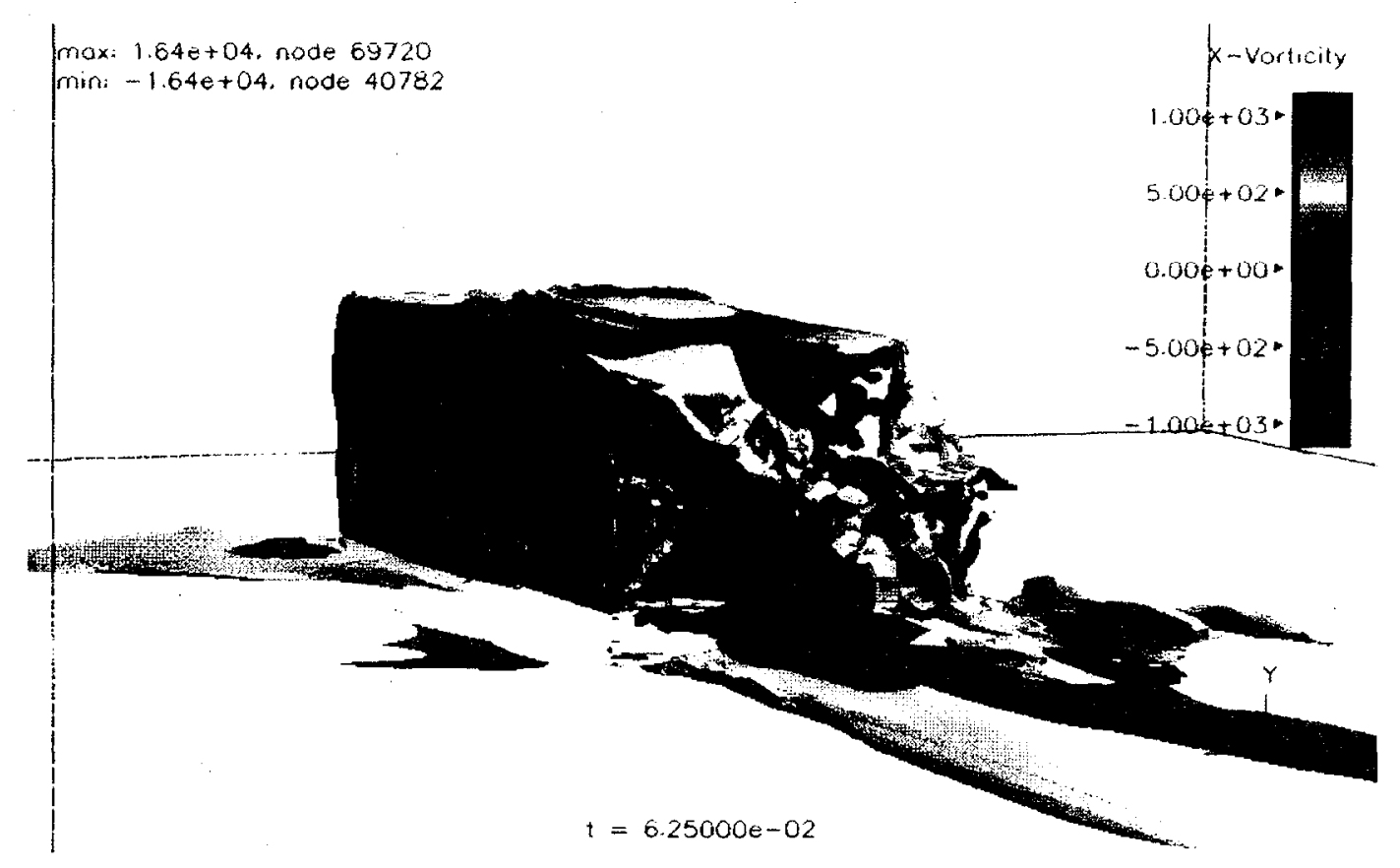




\section{Projected Sandia Funding for FY00}

\begin{tabular}{|c|c|c|c|c|c|c|c|}
\hline & FY98 & \multicolumn{4}{|c|}{ FY99 } & \multicolumn{3}{|c|}{ FY00 } \\
\hline & & Low & High & Received & Low & High & Received \\
\hline Old & $\$ 100 \mathrm{~K}$ & $\$ 150 \mathrm{~K}$ & $\$ 210 \mathrm{~K}$ & $\$ 80 \mathrm{~K}$ & $\$ 107 \mathrm{~K}$ & $\$ 275 \mathrm{~K}$ & \\
\hline New & & & & & $\$ 177 \mathrm{~K}$ & $\$ 345 \mathrm{~K}$ & \\
\hline
\end{tabular}

FTE cost for FY98: \$275K

FTE cost for FY99: $\$ 310 K$

FTE cost for FY00: \$326K (Projected) 
Truck Aerodynamics:

\title{
Large-Eddy Simulation (LES) using the Finite-Element Method (FEM)
}

\author{
Rose McCallen, Ph.D.
}

Lawrence Livermore National Laboratory

March 1999 


\section{We need advanced CFD tools to accurately predict drag effects for trucks.}

Flow is time dependent, $3 \mathrm{D}$ with a wide range of scales

Flapping recirculation zones

Thin boundary layers transition and separate

Flow tripped by head lamps, grab handles, etc.

Everything upstream effects what happens downstream

To reduce experimentation, accurate CFD with less empiricism is needed

Commercial CFD tools do not predict correct drag effects - per Industry Models have adjustable empirical parameters

Chosen approach

Large-eddy simulation (LES) using the finite element method (FEM) 
LES is a challenge but we have the experience and resources to succeed.

\section{Outline}

\section{Background}

Approach

Challenges

\section{Plan and Progress}

Formulation and implementation

Benchmarking

Truck simulation

Current Technology

RANS/LES hybrid - paper review 


\section{Large-eddy simulation provides a wealth of information}

and less empiricism.

Reynolds-averaged Navier-Stokes (RANS)

Many empirical parameters

Time-averaged solution

Backward-facing step: streamwise velocity

top wall

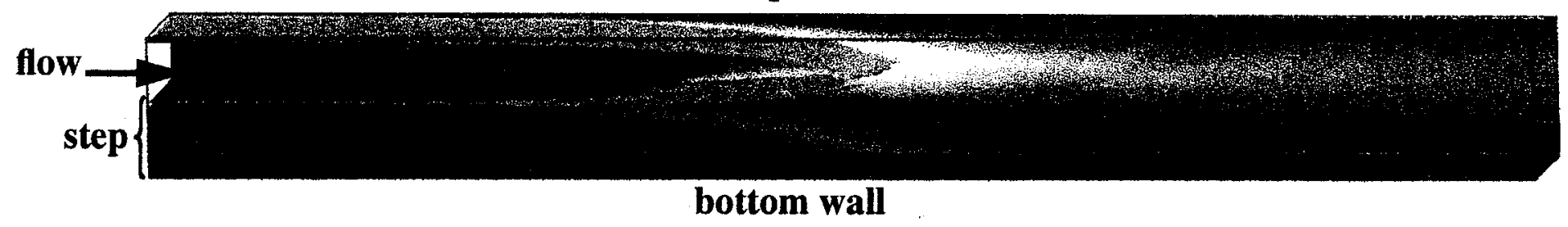

Large-eddy simulation (LES)

One empirical parameter $->$ less empiricism

3D, unsteady solution of vortex shedding

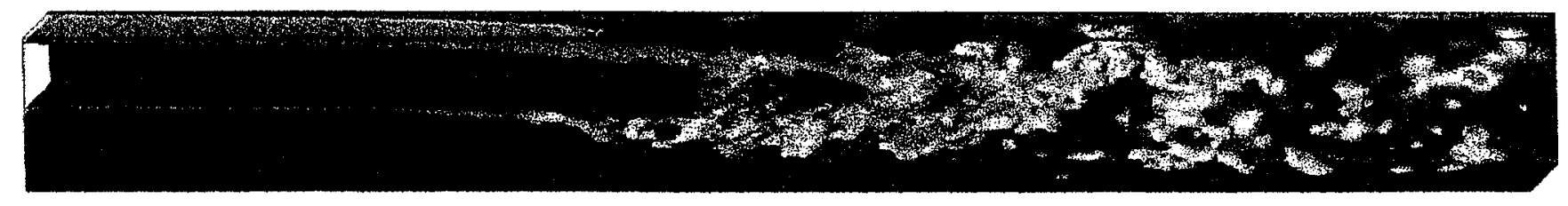




\section{The LES challenges are related to physical as well as numerical modeling.}

Boundary layers are too thin Can't resolve boundary layers - problem gets too big Wall approximations in development

Runtime too long

Evolution is over long time scales

Parallel computations/solvers required - in development

Analysis

Huge data sets

Visualization required - in development

Methods for testing convergence $(\mathrm{V} \& \mathrm{~V})$ in development

Significant development being done by LLNL programs. 


\section{The first year deliverable is to 'integrate' and develop the flow model and complete a demonstration problem.}

Milestone

FY99 incompressible flow demonstration

$\mathbf{R} \& \mathbf{D}$

Solver integration/parallelization

Turbulence modeling

Boundary conditions

Data analysis

Approach

Utilize existing methods, tools, resources, etc.

- Existing/tried formulation

- Smagorinsky SGS model for FY99

- Integrating existing codes

Take advantage of the Lab's infrastructure 
For the incompressible flow modeling we are taking advantage of existing methods and codes.

\begin{tabular}{|c|c|}
\hline $\begin{array}{c}\text { ALE3D } \\
\text { structural/thermal/chemistry/compressible-flow }\end{array}$ & \\
\hline \multicolumn{2}{|l|}{ Incompressible LES/FEM Code } \\
\hline Tasks & Status \\
\hline Establish compatibility and flexibility of the formulation & $\bar{J}$ \\
\hline Extract physics coding from existing code and modify for ALE3D & $\sqrt{ }$ \\
\hline Establish parallelization approach and implement & in progress \\
\hline Coding for input/output and postprocessing issues & in progress \\
\hline Benchmark testing & \\
\hline
\end{tabular}


The formulation is an established method, but solver implementation 'was' an issue.

Formulation, solution approach, and coupling

$$
\left(C^{T} M^{-1} C\right) P^{n}=C^{T} A^{n}
$$

Solve for pressure

$$
\begin{aligned}
& \text { where } A^{n}=M^{-1}\left[\left(K^{n}+N\left(u^{n}\right)\right) \underline{u}^{n}-F\left(u^{n}\right)\right] \\
& \underline{u}^{n+1}=\underline{u}^{n}-\delta t\left(A^{n}-M^{-1} C P^{n}\right)
\end{aligned}
$$

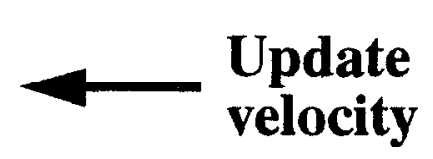

where

$$
C=\left[\begin{array}{l}
c_{i n(1)} \\
c_{i n(2)} \\
c_{i n(3)}
\end{array}\right] ; c_{i n(\alpha)}=\int_{\Omega} \psi_{n} \frac{\partial \phi_{i}}{\partial x_{\alpha}} M=\left[\begin{array}{ccc}
m_{i j} & 0 & 0 \\
0 & m_{i j} & 0 \\
0 & 0 & m_{i j}
\end{array}\right] ; m_{i j}=\int_{\Omega} \phi_{i} \phi_{j}
$$

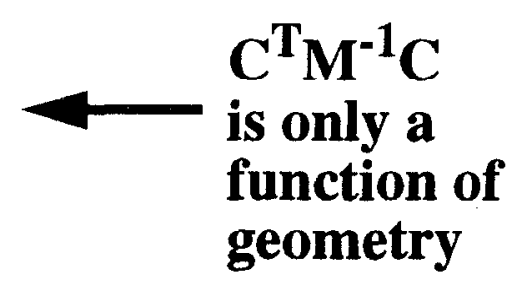

Matrix characteristics and solver

Piecewise constant pressure basis functions ('zone centered') Setting-up a row at a time!

Can't use solver's Finite Element Interface (FEI) 


\section{The $C^{T} M^{-1} C$ matrix is global and can't be constructed element-by-element.}

One-Dimensional Example

$$
\left(C^{T} M^{-1} C\right) P=r h s
$$

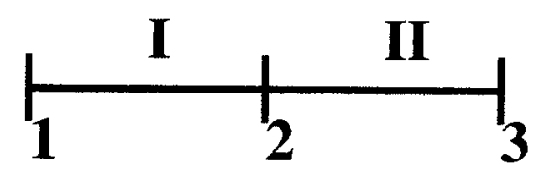

$\left[\begin{array}{ccc}c_{1 I} & c_{2 I} & 0 \\ 0 & c_{2 I I} & c_{3 I I}\end{array}\right]\left[\begin{array}{ccc}m_{1 I} & 0 & 0 \\ 0 & \left(m_{2 I}+m_{2 I I}\right) & 0 \\ 0 & 0 & m_{3 I I}\end{array}\right]\left[\begin{array}{cc}c_{1 I} & 0 \\ c_{2 I} & c_{2 I I} \\ 0 & c_{3 I I}\end{array}\right]\left[\begin{array}{l}P_{I} \\ P_{I I}\end{array}\right]=r h s$

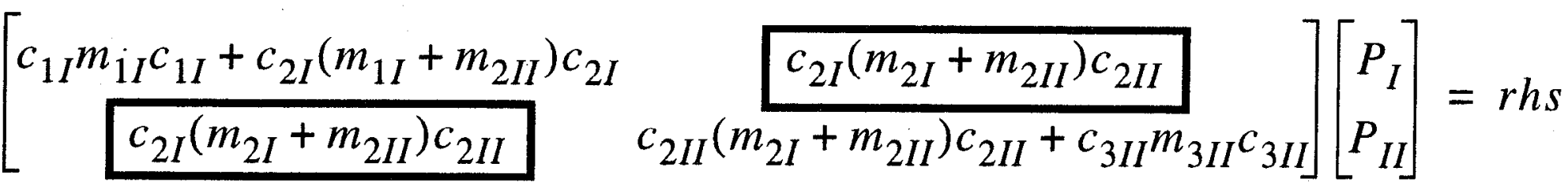

But, element-by-element formation looses the off-diagonal terms

$$
\begin{aligned}
& {\left[\begin{array}{ll}
c_{1} & c_{2}
\end{array}\right]\left[\begin{array}{cc}
m_{1} & 0 \\
0 & m_{2}
\end{array}\right]\left[\begin{array}{l}
c_{1} \\
c_{2}
\end{array}\right][P]=r h s} \\
& \left(c_{1} m_{1} c_{1}+c_{2} m_{2} c_{2}\right) P=r h s
\end{aligned}
$$

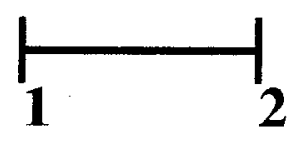

The matrix multiply must be done globally. 


\section{If the FEI performs the matrix multiply, the parallelization effort is significantly reduced.}

Parallelization outline

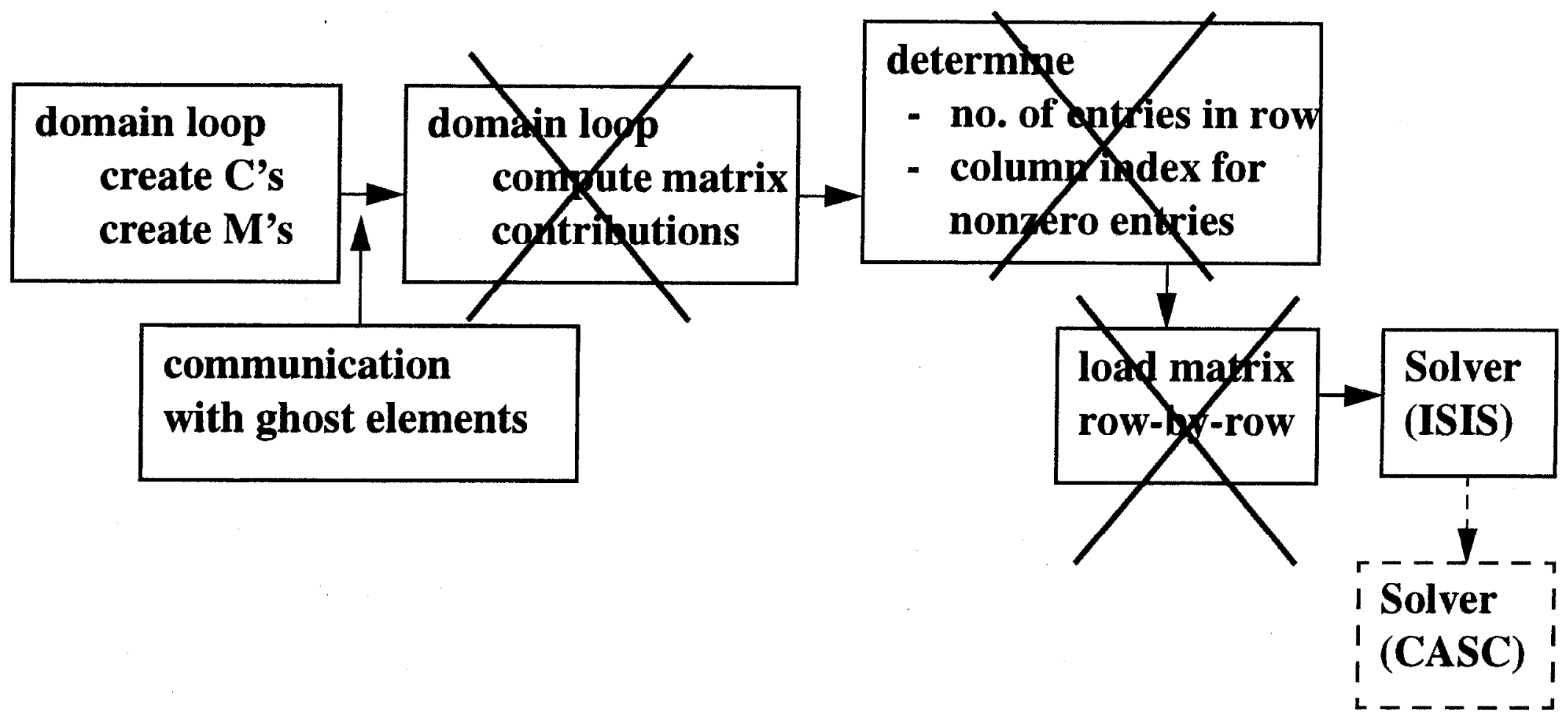




\section{A complete but expedient $V \& V$ method will be used.}

\section{Verification \& Validation}

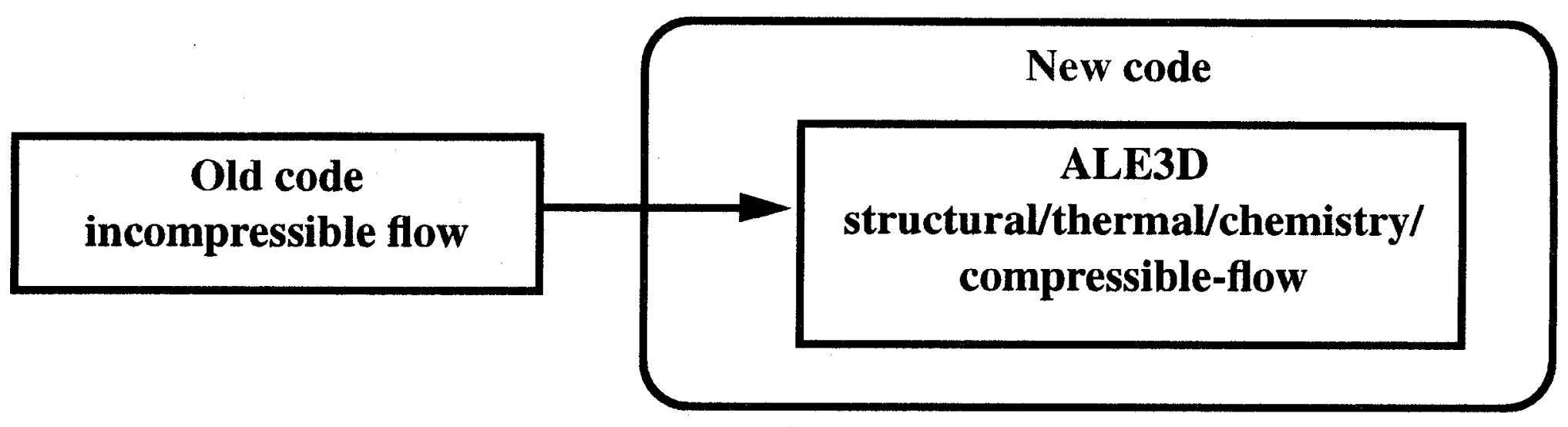

For an existing example problem (e.g., backward-facing step)

\begin{tabular}{|l|l|}
\hline \multicolumn{1}{|c|}{ Question } & \multicolumn{1}{c|}{ Cases } \\
\hline \hline Do old and new code agree? & compare to serial run with direct solver \\
\hline Does iterative solve work? & compare serial run with iterative and direct solve \\
\hline Does code run in parallel? & compare serial vs. parallel run for iterative solve \\
\hline
\end{tabular}




\section{Simulating the NASA 7'x10' wind tunnel experiment is the demonstration problem.}

Domain and boundary conditions are chosen to minimize grid size

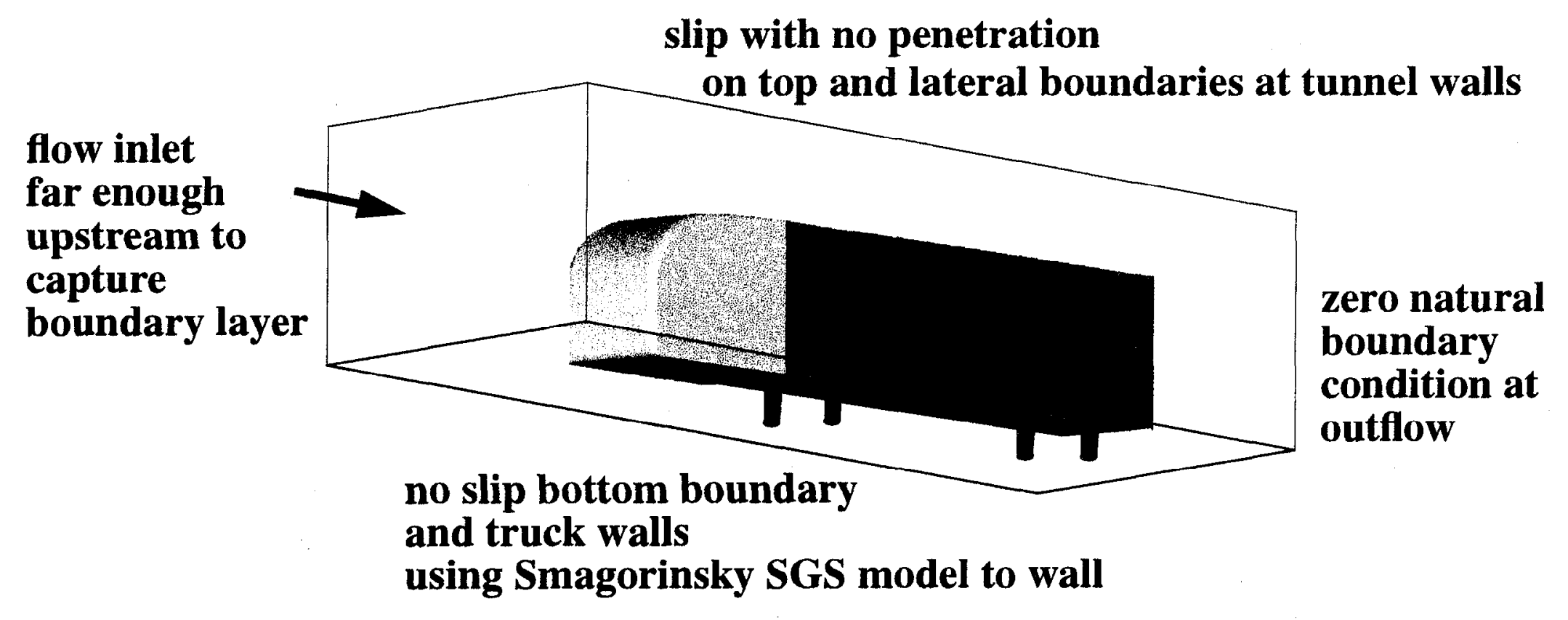


Compressible as well as incompressible simulations can be performed with an unstructured grid.

\section{Plan}

Compressible (Ma > .1)

NASA 7'x10' results for $\mathrm{Ma}=\mathbf{0 . 2 7}$

Incompressible $(\mathrm{Ma}<.1)$

NASA 7'x10' results for $\mathrm{Ma}<0.1$

USC results for $200,000<\operatorname{Re}<400,000$

Texas A\&M for $\operatorname{Re}=1,600,000$
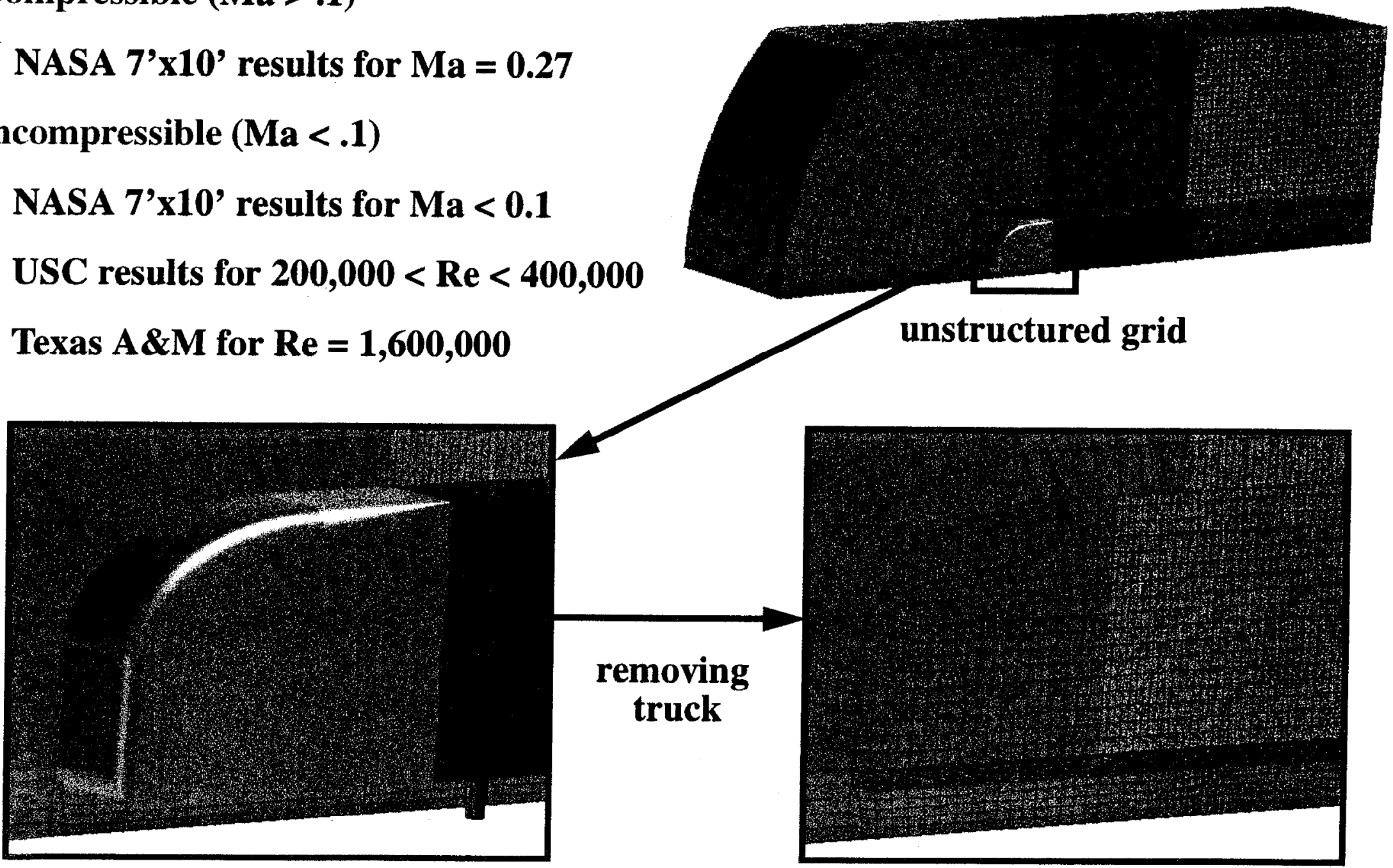

removing truck

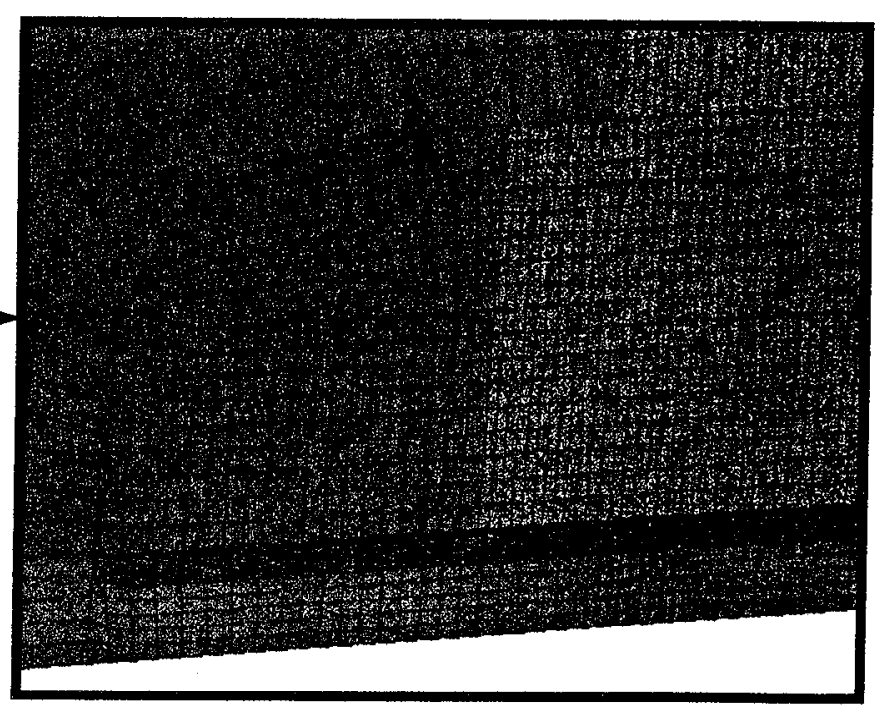




\section{Paper Review: LES is not feasible for attached flow (wings), but desirable for separating flow.}

Comments on the Feasibility of LES for Wings, and on a Hybrid RANS/LES Approach P.R. Spalart, W-H. Jou, M. Strelets, S.R. Allmaras, 1998.

Detached-Eddy Simulation (DES) method looks promising DES offers RANS in the boundary layers and LES after massive separation, within a single formulation.

For thin shear flows the grid resolution exceeds current capabilities (e.g., front/sides of cab) ... (for a wing) we find the need for $10^{11}$ grid points, under the most favorable conditions. Today, a calculation with $10^{8}$ points is impressive.

Coarse grids can be used in separation regions (e.g., truck wake) ... the most challenging flow regions for turbulence models "trained" in thin shear flows are the regions of massive separation ... driven by low-aspect-ratio features such as wheels and flap edges. This is where LES is most desirable ... would not require orders of magnitude in grid refinement.

Unstructured and adapted grids are required ... the grid coarsens as soon as possible outside the boundary layer; the irrotational region allows a grid spacing much larger than the boundary-layer eddies. 
Much has been done and much needs to be done.

\section{LES/FEM has advantages}

Accuracy with less empiricism

Built-in outflow conditions and unstructured grids

\section{LES/FEM has challenges}

Wall approximations

Parallel computations

Data analysis methods

Approach

Take advantage of existing methods and codes - keep it simple

\section{Current Technology}

DES method attempts to solve boundary layer resolution problem

LES/FEM is a challenge but we have the experience and resources to succeed. 


\title{
Vortex Methods for Flow Simulation
}

\author{
California Institute of Technology
}

\section{Essentials}

- Numerical technique to solve the Navier-Stokes Equations

- Suitable for Direct Simulation and Large-Eddy Simulation

- Uses vorticity (curl of the velocity) as a variable

- Computational elements move with the fluid velocity

\section{Advantages}

- Computational elements only where vorticity is non-zero

- No grid in the flow field

- Only 2D grid on vehicle surface

- Boundary conditions in the far field automatically satisfied 


\section{Vortex Method as a Flow Model}

\section{Previous limitations (1960s and 70s)}

- Inviscid model - dynamics of the boundary layer ignored

- Computationally limited - $O\left(N^{2}\right)$ operations per time step

- $N=$ only a few hundred to a few thousand computational elements feasible

- Dynamics of the wake and force coefficients dependent on adjustable parameters

Recent Developments (90s)

- Viscous effects treated accurately

- Fast Vortex Algorithm - $O(N)$ operations per time step

- $N=$ one to 10 million computational elements feasible

- Dense system of computational elements solves fluid equations

- Direct simulation for low Reynolds number

- Large-Eddy simulation for high Reynolds number

- Large-scale parallel computing 


\section{Treatment of Surface Vorticity}

\section{Standard Panel Method for N Panels}

- Low order accuracy - first order accurate

- Computationally and storage limited -O(N $\left.N^{2}\right)$ matrix elements computed and stored with $O\left(N^{2}\right)$ operations per time step

- Only $N=$ to feasible

\section{Advanced Panel Method}

- High accuracy - third order accurate

- Computationally efficient - $O(N)$ storage locations with $O\left(N^{/ 2}\right)$ operations per time step [can go to $O(N \quad N)$ or $O(N)$ ]

- $N=$ to no problem

- Triangular mesh with automatic refinement 


\section{Large-Eddy Simulation}

\section{Direct Simulation not Sufficient (1990s)}

- Direct Simulation possible for Reynolds No.= to (at parking speeds $-0.01 \mathrm{mph}$ )

- $N=\quad$ elements (approx 400,000 GBytes) required for Reynolds No.= (at highway speeds $-70 \mathrm{mph}$ )

\section{Large-Eddy Simulation Required}

- Treatment of small-scale (subgrid-scale) turbulence in the wake

- Treatment of small-scale turbulence in the boundary layers

- Treatment of fluidic actuators, blowing/suction, vortex generators and other flow control devices 


\section{Status / Future Work}

- Fast adaptive panel method

- Panel method interaction with outer flow via method similar to Verzicco

- Subgrid stress model for Large-Eddy Simulation

- Implementation of the Vortex Method for complex geometries 